\title{
Mathematical modeling of neurovascular coupling
}

Sebastian Sten

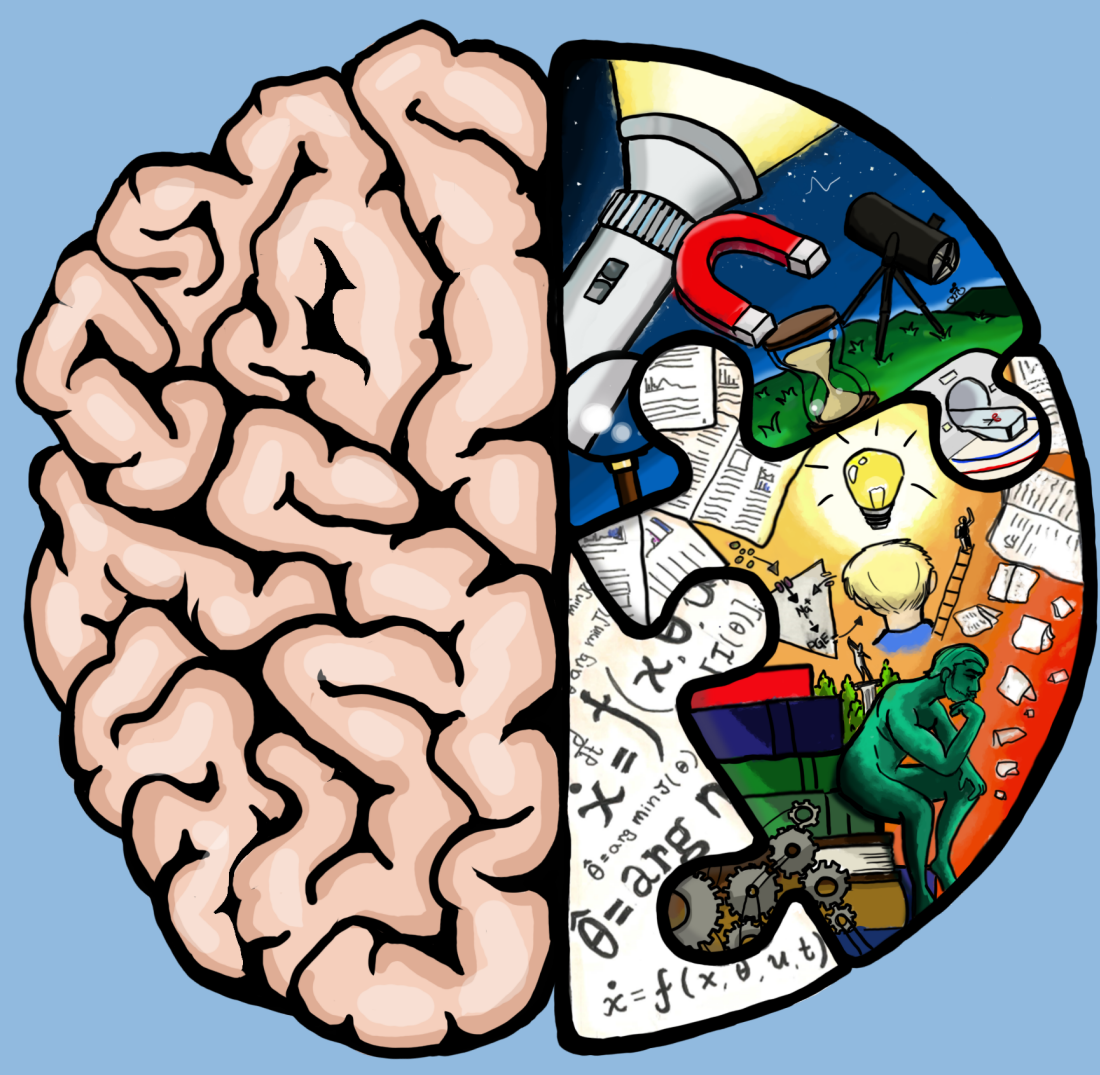

UINKÖPING 


\section{Mathematical modeling of neurovascular coupling.}

\section{Sebastian Sten}

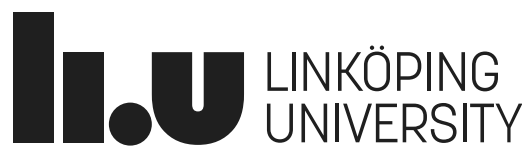

Linköping University

Department of Health, Medicine and Caring Sciences Division of Diagnostics and Specialist Medicine SE-581 83 Linköping, Sweden 


\section{Edition 1:1}

Cover: Understanding the complexity of the brain through Systems Biology. Illustrated by Christian Simonsson.

(C) Sebastian Sten, 2020

ISBN 978-91-7929-838-8

ISSN 0345-0082

URL http://urn.kb.se/resolve?urn=urn:nbn:se:liu:diva-167806

Published articles have been reprinted with permission from the respective copyright holder.

Typeset using $\mathrm{X}_{\mathrm{H}} \mathrm{T}_{\mathrm{E}} \mathrm{X}$

Printed by LiU-Tryck, Linköping 2020 


\section{Main supervisor}

\section{Maria Engström}

Professor, Ph.D.

Department of Health, Medicine and Caring Sciences

Center for Medical Image Science and Visualization (CMIV)

Linköping University

\section{Co-supervisors}

\section{Gunnar Cedersund}

Associate professor, Ph.D.

Department of Biomedical Engineering

Linköping University

\section{Fredrik Elinder}

Professor, Ph.D.

Department of Biomedical and Clinical Sciences

Linköping University

\section{Opponent}

\section{Gaute T. Einevoll}

Professor, Ph.D.

Department of Physics, University of Oslo, Oslo, Norway

Faculty of Science and Technology, Norwegian University of Life Sciences, Ås, Norway 



\section{POPULÄRVETENSKAPLIG SAMMANFATTNING}

Hjärnan kräver, för att bevara sin normala funktion, en kontinuerlig tillströmning av metaboliter så som syre och glukos, som bärs och levereras av blodomloppet. När ett hjärnområde aktiveras ökar förbrukningen av dessa metaboliter kvickt. Detta kompenseras snabbt för igenom att blodtillförseln till hjärnområdet ökar, vilket temporärt ökar syresättningen av blodet i det aktiverade hjärnområdet under flera sekunder, långt efter att aktiviteten avtagit. Detta fenomen utgör grunden för flera av de icke-invasiva tekniker som idag används för att kartlägga hjärnans funktion i både människor och djur. Ett exempel är funktionell magnetresonanstomografi (fMRI) som mäter lokala förändringar av syrehalten i hjärnan och använder detta som en markör för att lokalisera aktiverade hjärnområden. Användningen av fMRI har revolutionerat hjärnforskningen sedan den introducerades för 30 år sedan, men då tekniken indirekt mäter hjärnaktivitet genom syrehalten i blodet är det viktigt att förstå den serie av händelser som sker mellan ökad hjärnaktivitet och ökad blodtillförsel till hjärnområdet: den neurovaskulära kopplingen.

Den neurovaskulära kopplingen förbinder den elektriska aktiviteten i nervceller med lokala förändringar i blodflöde, blodvolym och metabolism av syre, genom ett komplext biokemiskt system av olika typer av hjärnceller som utsöndrar substanser som påverkar blodkärlen. För att uppnå en ökad förståelse för hur sådana komplexa biologiska system fungerar kan man använda sig av matematisk modellering och skapa en datormodell över systemet, som är en huvudgren inom forskningsområdet Systembiologi. I denna avhandling har vi utvecklat en serie av matematiska modeller som beskriver och undersöker de intracellulära biokemiska signalvägar som den neurovaskulära kopplingen består av, genom att använda oss av olika typer av experimentell data insamlat i flera olika arter: möss, apor och människor.

Artikel 1 undersöker två av de vanligast förekommande hypoteserna som beskriver den neurovaskulära kopplingen. Vi visar med hjälp av modellerna att varje hypotes var för sig inte kan förklara fMRI-data insamlad i människa, men en kombination av de två hypoteserna kan. Denna kombinerade modell kan även korrekt förutsäga hur mätdata bör se ut för olika fall den aldrig tidigare fått se.

Artikel 2 utökar denna modell till att även beskriva scenarion där syrehalten i blodet minskar på grund av att aktiviteten i hjärnområdet hämmas. Denna hämning fyller en viktig funktion då den reglerar aktiviteten i olika hjärnområden så att andra hjärnområden inte hindras från att utföra olika uppgifter.

Artikel 3 beskriver en ny typ av matematisk modell för den neurovaskulära kopplingen, som kan särskilja olika nervcellers bidrag till regleringen av blodkärlen. Detta är möjligt igenom experimentell data som genererats med hjälp av optogenetik, där en ljuskänslig jonkanal uttrycks i specifika typer av nervceller i möss. Med hjälp av en ljuspuls kan man då aktivera olika typer av nervceller var för sig. Modellen kan även beskriva hur narkosmedel förändrar den funktionella kärlregleringen, samt förutsäga effekten av olika typer av biokemiska hämmare.

I Artikel 4 utökas denna modell till att kunna beskriva och förutsäga experimentell data från de allra flesta tillgängliga mätmetoder som används för att undersöka den neurovaskulära kopplingen. Denna modell bidrar med insikter om hur olika typer av observerade fenomen uppstår på olika nivåer av signaleringskedjan.

Denna nya modellbaserade förståelse kring den neurovaskulära kopplingen ger möjlighet till en djupare analys av experimentell data relaterad till hjärnans funktion, med både kliniska och forskningsrelaterade tillämpningar. 


\begin{abstract}
The brain is critically dependent on the continuous supply of oxygen and glucose, which is carried and delivered by blood. When a brain region is activated, metabolism of these substrates increases rapidly, but is quickly offset by a substantially higher increase in blood flow to that region, resulting in a brief oversupply of these substrates. This phenomenon is referred to as functional hyperemia, and forms the foundation of functional neuroimaging techniques such as functional Magnetic Resonance Imaging (fMRI), which captures a Blood Oxygen Level-Dependent (BOLD) signal. fMRI exploits these BOLD signals to infer brain activity, an approach that has revolutionized the research of brain function over the last 30 years. Due to the indirect nature of this measure, a deeper understanding of the connection between brain activity and hemodynamic changes - a neurovascular coupling (NVC) - is essential in order to fully interpret such functional imaging data. NVC connects the synaptic activity of neurons with local changes in cerebral blood flow, cerebral blood volume, and cerebral metabolism of oxygen, through a complex signaling network, consisting of multiple different brain cells which release a myriad of distinct vasoactive messengers with specific vascular targets. To aid with this complexity, mathematical modeling can provide vital help using methods and tools from the field of Systems Biology. Previous models of the NVC exist, conventionally describing quasi-phenomenological steps translating neuronal activity into hemodynamic changes. However, no mechanistic mathematical model that describe the known intracellular mechanisms or hypotheses underlying the NVC, and which can account for a wide variety of NVC related measurements, currently exists. Therefore, in this thesis, we apply a Systems Biology approach to develop such intracellular mechanisms based models using in vivo experimental data consisting of different NVC related measures in rodents, primates, and humans.
\end{abstract}

Paper I investigates two widely discussed hypotheses describing the NVC: the metabolic feedback hypothesis, and the vasoactive feed-forward hypothesis. We illustrate through multiple model rejections that only a model describing a combination of the two hypotheses can capture the qualitative features of the BOLD signal, as measured in humans. This combined model can describe data used for training, as well as predict independent validation data not previously seen by the model before.

Paper II extends this model to describe the negative BOLD response, where the blood oxygenation drops below basal levels, which is commonly observed in clinical and cognitive studies. The model explains the negative BOLD response as the result of neuronal inhibition, describing and adequately predicting experimental data from two different experiments.

In Paper III, we develop a first model including the cell-specific contributions of GABAergic interneurons and pyramidal neurons to functional hyperemia, using data of optogenetic and sensory stimuli in rodents for both awake and anesthesia conditions. The model captures the effect of the anesthetic as purely acting on the neuronal level if a Michaelis-Menten expression is included, and it also correctly predicts data from experiments with different pharmacological inhibitors.

Finally, in Paper IV, we extend the model in Paper III to describe and predict a majority of the relevant hemodynamic NVC measures using data from rodents, primates, and humans. The model suggests an explanation for observed bi-modal behaviors, and can be used to generate new insights regarding the underpinnings of other complicated observed behaviors. This model constitutes the most complete mechanistic model of the NVC to date.

This new model-based understanding opens the door for a more integrative approach to the analysis of neuroimaging data, with potential applications in both basic science and in the clinic. 


\section{List of Included Publications and Manuscripts}

This thesis is based on the following papers, referred to in the text by their Roman numerals (I-IV).

I Karin Lundengård, Gunnar Cedersund, Sebastian Sten, Felix Leong, Alexander Smedberg, Fredrik Elinder, Maria Engström. Mechanistic Mathematical Modeling Tests Hypotheses of the Neurovascular Coupling in fMRI. PLoS Comput Biol, 2016; 12: e1004971. https://doi.org/10.1371/journal.pcbi.1004971

II Sebastian Sten, Karin Lundengård, Suzanne Tyson Witt, Gunnar Cedersund, Fredrik Elinder, Maria Engström. Neural inhibition can explain negative BOLD responses: A mechanistic modelling and fMRI study. Neuroimage, 2017; 158: 219-231. https://doi.org/10.1016/j.neuroimage.2017.07.002

III Sebastian Sten, Fredrik Elinder, Gunnar Cedersund ${ }^{*}$, Maria Engström $^{*}$. A quantitative analysis of cell-specific contributions and the role of anesthetics to the neurovascular coupling. Neuroimage, 2020; 215:116827. https://doi.org/10.1016/j.neuroimage.2020.116827

IV Sebastian Sten, Henrik Podéus, Nicolas Sundqvist, Fredrik Elinder, Maria Engström*, Gunnar Cedersund ${ }^{*}$. A multi-data based quantitative model for the neurovascular coupling in the brain. In manuscript.

* Authors contributed equally 



\section{Acknowledgments}

My thesis work has arisen with contributions and help from many people to whom I would like to express my gratitude.

First of all, I would like to thank my primary advisor, Maria Engström. I'm grateful that you believed in me as a barely half-finished engineering student, and took me on as an intern and later PhD-student in your group. I appreciate that you have allowed me to pursue my own work, and ever so slightly nudging me back into the right trajectory when I have been led astray by my own devices.

I'm very thankful for my co-advisors. Starting with Gunnar Cedersund, you introduced (or lured) me into the world of science. I am thankful that you openly took me into your group, and everything you've taught me over the years. Fredrik Elinder, for sharing your experience and wisdom in not only how to produce good science, but equally important how to structure and write good science. The brief collaboration on an updated ion-channel model for your teaching course was a fun and fruitful project.

A special thanks to all of my unofficial mentors, who gracefully welcomed me and taught me a great deal of things during these past years: Rozalyn Simon, Elin Nyman, Rikard Johansson, Karin Lundengård, Natasha Morales Drissi, Helene Veenstra and Suzanne Tyson Witt.

Thank you, all the people, in the systems biology group over at IMT. Thanks for all the good times and laughs: William Lövfors, Christian Simonsson, Nicolas Sundqvist, Tilda Herrgårdh, and many other past and present students. A special thanks to Christian who gracefully illustrated the front page symbol.

I'm grateful for the support from the unique research environment over at CMIV, along with all of the PhD-students in the CMIV research school for all the research presentations.

A big thanks to my parents, Anna \& Mikael, and my sister Josephine, for all of the support over these years.

Finally, to my love Anna, I'm grateful for your unending support.

Sebastian Sten

Linköping, July 2020 
20-HETE 20-hydroxyeicosatetraenoic acid.

AA Arachidonic Acid.

AMPA $\quad \alpha$-amino-3-hydroxy-5-methyl-4-isoxazolepropionic Acid.

ATP Adenosine Triphosphate.

$\mathrm{BK}_{\mathrm{Ca}} \quad$ Large-conductance calcium activated $\mathrm{K}^{+}$Channels.

BOLD Blood Oxygen Level-Dependent.

cAMP Cyclic Adenosine Monophosphate.

CBF Cerebral Blood Flow.

CBV Cerebral Blood Volume.

cGMP Cyclic Guanosine Monophosphate.

ChR2 ChannelRhodopsin-2.

$\mathrm{CMRO}_{2}$ Cerebral Metabolic Rate of Oxygen.

COX Cyclooxygenase.

CYP Cytochrome P450.

DAEs Differential Algebraic Equations.

EETs Epoxyeicosatrienoic acids.

FIM Fisher Information Matrix.

fMRI functional Magnetic Resonance Imaging.

GABA $\quad \gamma$-aminobutyric Acid.

GLM General Linear Model.

HRF Hemodynamic Response Function.

IP3 R Inositol-1,4,5-trisphosphate Receptor.

$\mathrm{K}_{\mathrm{IR}} \quad$ Inward-rectifying $\mathrm{K}^{+}$channel.

LFP Local Field Potential.

MCMC Markov Chain Monte Carlo.

mGluR metabotropic Glutamate Receptors.

MRI Magnetic Resonance Imaging.

NIRS Near-Infrared Spectroscopy.

NMDA N-methyl-D-aspartate.

nNOS neuronal Nitric Oxide Synthase.

NO Nitric Oxide.

NPY Neuropeptide Y.

NPY1R NPY receptor Y1.

NVC Neurovascular Coupling.

ODEs Ordinary Differential Equations.

OG Optogenetic.

$\mathrm{PGE}_{2} \quad$ Prostaglandin $\mathrm{E}_{2}$.

PL Profile Likelihood.

PPL Prediction Profile Likelihood.

RF Radio Frequency.

RK4 Runge-Kutta 4th order.

SA Simulated Annealing.

SEM Standard error of the mean.

SOM Somatostatin.

SSR Sum of Squares Residual.

SSTR Somatostatin receptors.

$\mathrm{V}_{M} \quad$ Membrane potential.

VASO Vascular-Space-Occupancy.

VIP Vasointestinal Peptide.

VPAC VIP/PACAP receptor.

VSM Vascular Smooth Muscle. 


\section{Mathematical Notations}

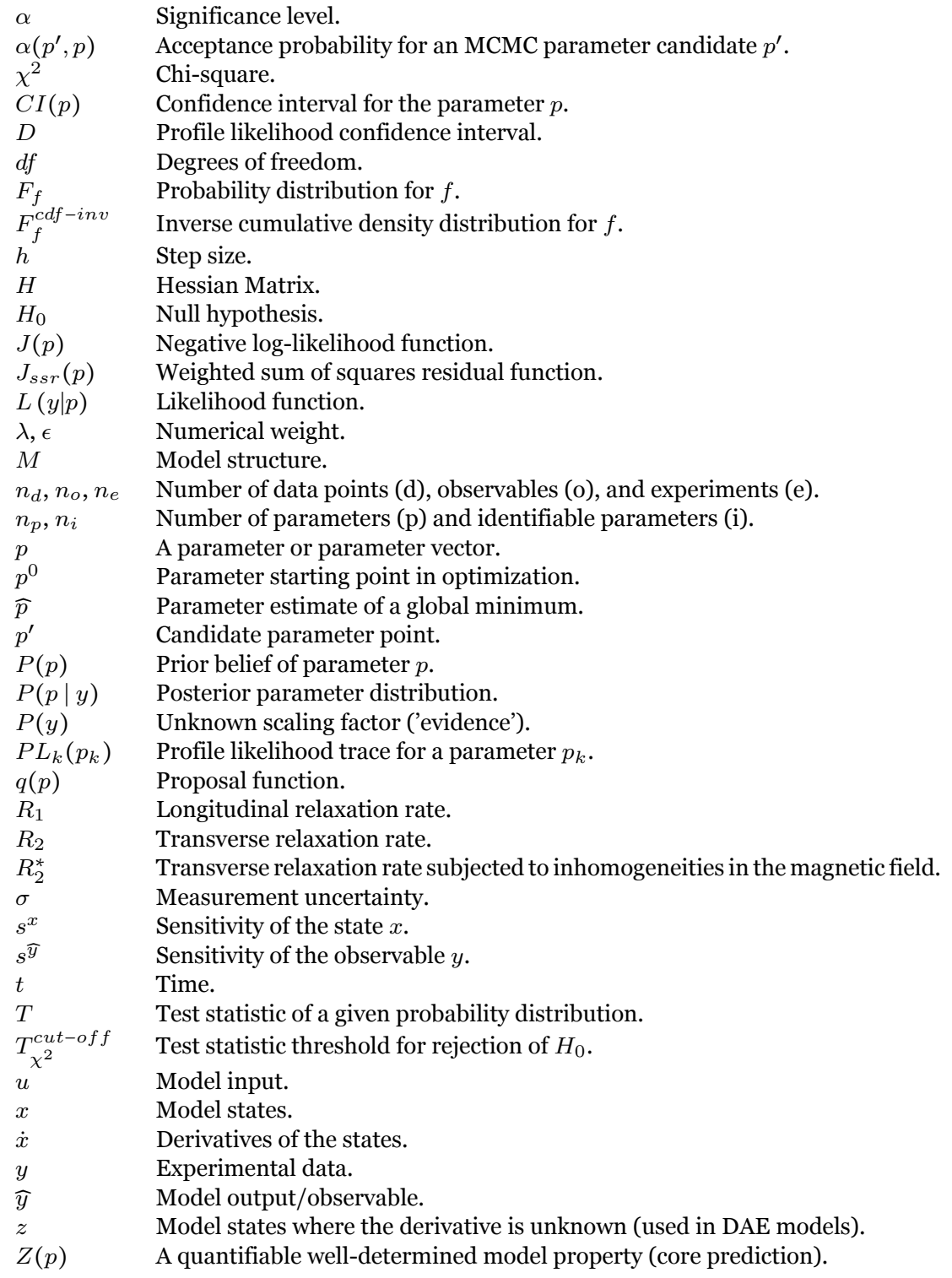





\section{Contents}

Abstract v v

List of Included Original Research vii

$\begin{array}{ll}\text { Acknowledgments } & \text { ix }\end{array}$

Abbreviations $\quad \mathbf{x}$

Mathematical Notations $\quad$ xi

Contents $\quad$ xiii

$\begin{array}{ll}\text { List of Figures } & \text { Xv }\end{array}$

List of Tables $\quad$ xvii

1 Introduction 1

1.1 Thesis outline $\ldots \ldots \ldots \ldots 2$

2 Background $\mathbf{3}$

2.1 Anatomy and physiology of the mammal brain . . . . . . . . . 3

2.2 A short introduction to the neurovascular coupling . . . . . . . 9

2.3 Systems Biology . . . . . . . . . . . . . . . . . . . . . 12

2.4 Aims and scope .................... 14

3 Neurovascular Coupling $\quad \mathbf{1 5}$

3.1 The discovery of a NVC . . . . . . . . . . . . . . . . 15

3.2 Feed-forward hypothesis governing the NVC . . . . . . . . . 17

3.3 In vivo measurement techniques for capturing NVC responses 21

3.4 Pharmacological modulations of the NVC . . . . . . . . 26

4 Mathematical Modeling in Systems Biology 29

4.1 Iterative cycle of mathematical modeling . . . . . . . . . . . 30

4.2 Ordinary and algebraic differential equations ....... 30

4.3 Model simulation .................... 31 
4.4 Likelihood functions . . . . . . . . . . . . . . . . . 34

4.5 Optimization - minimization of the likelihood function . . . 37

4.6 Assessing goodness of fit using statistical tests . . . . . . . . 42

4.7 Identifiability of parameters and uncertainty analysis . . . . 44

4.8 The necessity of validation data $\ldots \ldots \ldots \ldots \ldots \ldots$

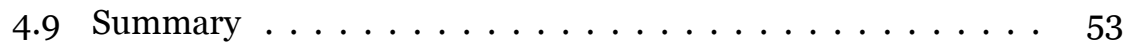

5 Results

5.1 Paper I: evaluating two of the most widely discussed hypotheses underpinning the NVC . . . . . . . . . . . 55

5.2 Paper II: negative BOLD responses - the role of inhibition . . 58

5.3 Paper III: assessing cell-specific contributions to the NVC and the role of anesthesia . . . . . . . . . . . 60

5.4 Paper IV: the final model can describe most relevant NVC mea-

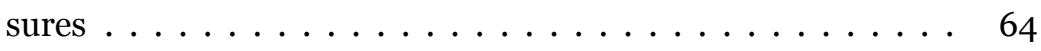

6 Discussion and concluding remarks 67

6.1 Summary of main findings and conclusions . . . . . . . . 67

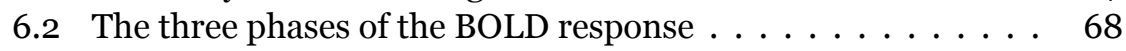

6.3 The three vascular compartments $\ldots \ldots \ldots \ldots \ldots \ldots$

6.4 Negative BOLD . . . . . . . . . . . . . . . . . . 72

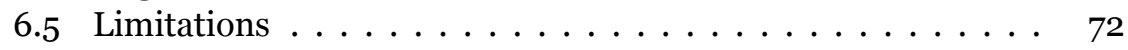

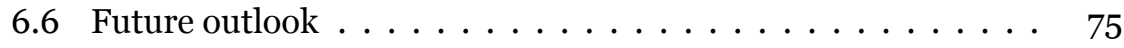

$\begin{array}{ll}\text { Bibliography } & 79\end{array}$

$\begin{array}{ll}\text { Paper I } & 109\end{array}$

$\begin{array}{ll}\text { Paper II } & 139\end{array}$

$\begin{array}{lr}\text { Paper III } & 155\end{array}$

$\begin{array}{ll}\text { Paper IV } & 169\end{array}$ 


\section{List of Figures}

2.1 The first recorded intracellular action potential . . . . . . 4

2.2 Neurons underlie the electrical signaling in the brain . . . . . . . 5

2.3 Axial view of the human brain . . . . . . . . . . . 6

2.4 The layered column-like structure of the cortex $\ldots \ldots \ldots$. . . 7

2.5 The division of the cortex into Brodmann areas . . . . . . . 8

2.6 Short overview of NVC . . . . . . . . . . . . . . 9

2.7 Correlational fMRI analysis vs mechanistic model-based analysis 11

3.1 The first proof of a NVC by Mosso in $1881 \ldots \ldots \ldots \ldots$

3.2 Overview of most vasoactive pathways found in the NVC . . . . . 19

3.3 fMRI: from image to statistical maps . . . . . . . . . . . . 23

3.4 Simple introduction to optogenetics . . . . . . . . . . 26

3.5 The effects of anesthetics on observed hemodynamic responses . 27

4.1 The iterative cycle of mathematical modeling . . . . . . . . 31

4.2 Geometric representation of the 4th order Runge-Kutta formula . 33

4.3 Comparison between 4th order Runge-Kutta and a modern ODE solver within the SUNDIALS package . . . . . . . . . 33

4.4 Computational time of likelihood gradient calculation with respect to the number of parameters using three different methods . . . 37

4.5 Hypothetical likelihood landscape for a one parameter system . . 38

4.6 Gradient descent vs Newton method for a two parameter landscape 40

4.7 Simple practical illustration of the Simulated Annealing algorithm 41

4.8 Simple practical illustration of the Scatter Search algorithm . . . . 43

4.9 The three cases of parameter identifiability . . . . . . . . . . . 47

4.10 Comparison between an MCMC and a PL analysis for a five parameter model of liver contrast agent uptake . . . . . . . . 50

4.11 Polynomial example of over-fitting . . . . . . . . . . . 52

4.12 Illustrating the use of validation to remedy over-fitting . . . . . 53

5.1 Summary of the two common hypotheses underpinning the NVC 55

5.2 The rejection of the metabolic feedback hypothesis . . . . . 56 
5.3 A combined model can describe both data used for estimation and predict independent validation data . . . . . . . . . . 57

5.4 Archetypal positive and negative BOLD responses . . . . . . . 58

5.5 Model estimation and analysis for positive and negative eventrelated BOLD responses . . . . . . . . . . . . . . 59

5.6 Introductory figure to Paper III . . . . . . . . . . . . . . . 61

5.7 Model estimation and predictions of arteriolar response data from awake and anesthetized mice . . . . . . . . . . . 62

5.8 The model suggests an explanation for the underlying dynamic difference between awake and anesthetized animals in the case of OG stimulation . . . . . . . . . . . . . . 63

5.9 Overview of Paper IV . . . . . . . . . . . . . . . . 64

5.10 Model explanation of the transition from an one-peak to a twopeak arteriolar response . . . . . . . . . . . 66 


\section{List of Tables}

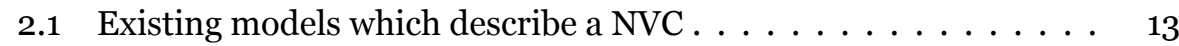





\section{1}

\section{Introduction}

"Men ought to know that from the brain, and from the brain only, arise our pleasures, joys, laughter and jests, as well as our sorrows, pains, griefs and tears. Through it, in particular, we think, see, hear, and distinguish the ugly from the beautiful, the bad from the good, the pleasant from the unpleasant . . . It is the same thing which makes us mad or delirious, inspires us with dread and fear, whether by night or by day, brings sleeplessness, inopportune mistakes, aimless anxieties, absent-mindedness, and acts that are contrary to habit."

- Hippocrates, Fifth Century B.C.E

Our brain - a slimy wrinkled mound of grayish tissue - is the vessel through which our awareness of the external (i.e., physical surroundings) and internal existence emerge: consciousness. The brain integrates a nonstop stream of information stemming from our sensory organs and presents this information in a coherent and unified manner, through which one can interpret and manipulate the physical environment. The human brain has evolved to possess higher-order cognitive functions such as abstract thinking and problem solving, which have aided the rapid evolution of mankind. However, how the higher-order cognitive functions of the brain emerge from the brain's cellular, molecular, and anatomical structure is still not well understood. In search of answers to this question, scientists have during the last century, step by step, been able to probe and generate large amounts of experimental data from the brain, due to a series of major breakthroughs in biomedical imaging and other experimental techniques. In parallel to these experimental breakthroughs, the rise of computational power during the last decades has laid the foundation of artificial intelligence, which is heavily influenced by the naturally occurring design of the brain. This design is made up of an intangible weave of connections between neurons in the form of electrical synapses, which continuously change over time. Due to this complexity, 
the brain relies on a constant supply of biological necessities such as glucose and oxygen, which are carried and delivered by blood. This doctoral thesis revolves around modeling how the brain manages to balance the supply and demand for blood by actively regulating the vasculature as a function of neuronal activity.

\subsection{Thesis outline}

Beyond this introductory chapter, this thesis is divided into five additional chapters.

Chapter 2 gives a brief historical background and introduction to neuroscience and Systems Biology, which leads up to the research questions which are studied in this thesis.

Chapter 3 provides background information regarding the current state-ofthe-art understanding of the NVC and some of its pertaining aspects.

Chapter 4 introduces the reader to the core methodological concepts of Systems Biology and mathematical modeling, which is extensively applied throughout the thesis.

Chapter 5 summarizes the main findings and how they fit into the overall picture.

Finally, Chapter 6 provides some concluding remarks and thoughts on the future outlook and potentials of the findings. 


\section{2}

\section{Background}

The following chapter aims to introduce the necessary background information to the reader. This information entails a basic overview of neuroscience with an emphasis on the basic mechanism of balancing the supply and demand of blood in the brain, and a brief introduction to the interdisciplinary field of Systems Biology. With these concepts in place, the chapter ends with introducing the research questions that this thesis revolves around.

\subsection{Anatomy and physiology of the mammal brain}

This section provides a brief introduction to the anatomy and physiology of the mammal brain. The section begins with the basic building block of information in the brain - action potentials - and then continues upwards in scale, through neurons, the cortex, and finally to the whole-brain perspective. This is a short introduction to these concepts, and for a more comprehensive introduction to neuroscience in general, I refer the reader to [1].

\section{The language of information in the brain - the action potential}

The language of information in the brain is based on the transmission of rapid electrical impulses, commonly known as action potentials. The first recorded intracellular action potential (see Fig. 2.1, left) was captured in squid giant axons in 1939 by the Nobel prize recipients A.L Hodgkin and A.F Huxley [2]. The archetypal action potential is depicted in Figure 2.1 (right), and it exhibits three distinct phases: 1) depolarization, where the potential rapidly rise; 2) repolarization, where the potential abruptly shoots back downwards, and finally, 3) hyper-polarization, where the potential remains below the resting potential before slowly re-establishing the resting potential. The electrical 

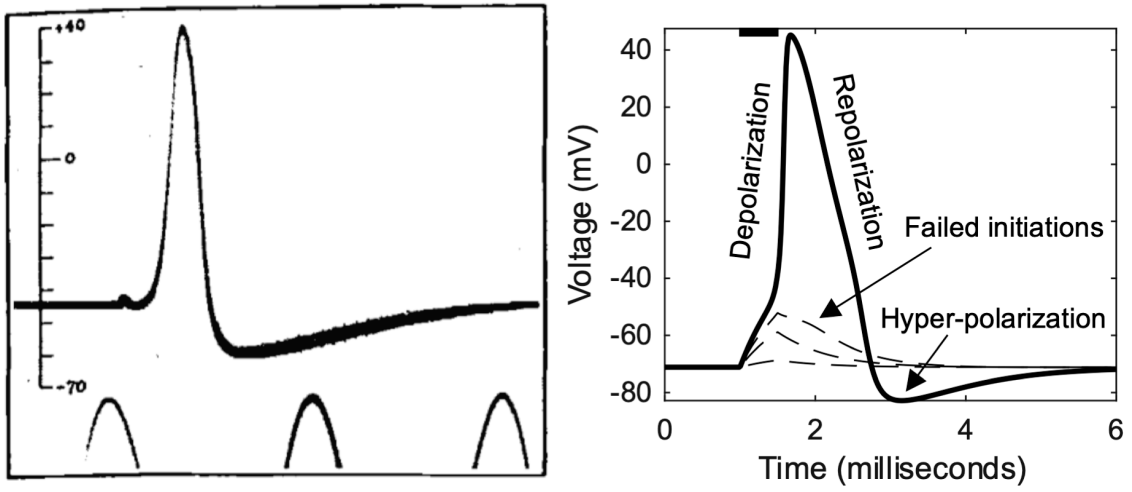

Figure 2.1: The first intracellular recording of an action potential (left), captured in the squid giant axon. The vertical scale shows the potential of the internal electrode in millivolts, using the extracellular seawater as zero potential. The sinusoidal wave peaks in the lower part of the figure correspond to a $500 \mathrm{~Hz}$ timing signal. Figure taken from [2] with permission. Right: The three phases of an action potential: rapid depolarization, repolarization, and finally, a period of hyper-polarization before returning to baseline. The different baseline potential values between left and right are due to different extracellular medium.

stimulation needs to be strong enough to initiate the response, meaning that the action potential can be viewed as an 'all-or-nothing' response.

\section{Neurons}

Action potentials originate from excitable cells, which express voltage-gated ion channels that are embedded in the lipid plasma membrane of the cell. The excitable cells are neurons which generate, receive, process, and transmit action potentials throughout the brain (Fig. 2.2A). The notion that the brain is comprised of individual neurons was first proven in the late 1800 s, long after the idea that living tissue consists of individual cells was accepted. Initially, the brain was thought to be an exception; that the brain instead was made up of a continuous protoplasmic network, as hypothesized by the anatomist Joseph von Gerlach $(1820-1896)$ in 1871 [3, 4]. This hypothesis was, in part, a result of methodological limitations, as neurons exhibit highly irregular shapes and long thin processes extending from the nucleus (Fig. 2.2). This made neurons hard to clearly distinguish using the only technique available at the time: conventional light microscopy. This experimental limitation was overcome by Camillo Golgi (1843-1926), who developed a new cellular staining technique in 1873: la reazione nera or Golgi's method [5, 6]. Golgi's method allowed for these small details to be clearly seen in the preparation. This technique was extensively used by both Golgi and Santiago Ramón y Cajal (1852-1934) to produce detailed drawings of neurons in many brain regions or different species (Fig. 2.2B) [7]. Golgi and Cajal were later awarded the Nobel prize in 1906 for these discoveries. These seminal 
drawings laid the foundation for the 'neuron doctrine', which states that the brain is made up of discrete individual cells ('neurons') which are not structurally connected, but communicate through synapses (see [7]). A synapse is a site of information relay, which allows for an electrical signal to be relayed between two neurons through the use of chemical neurotransmitters (discussed below).
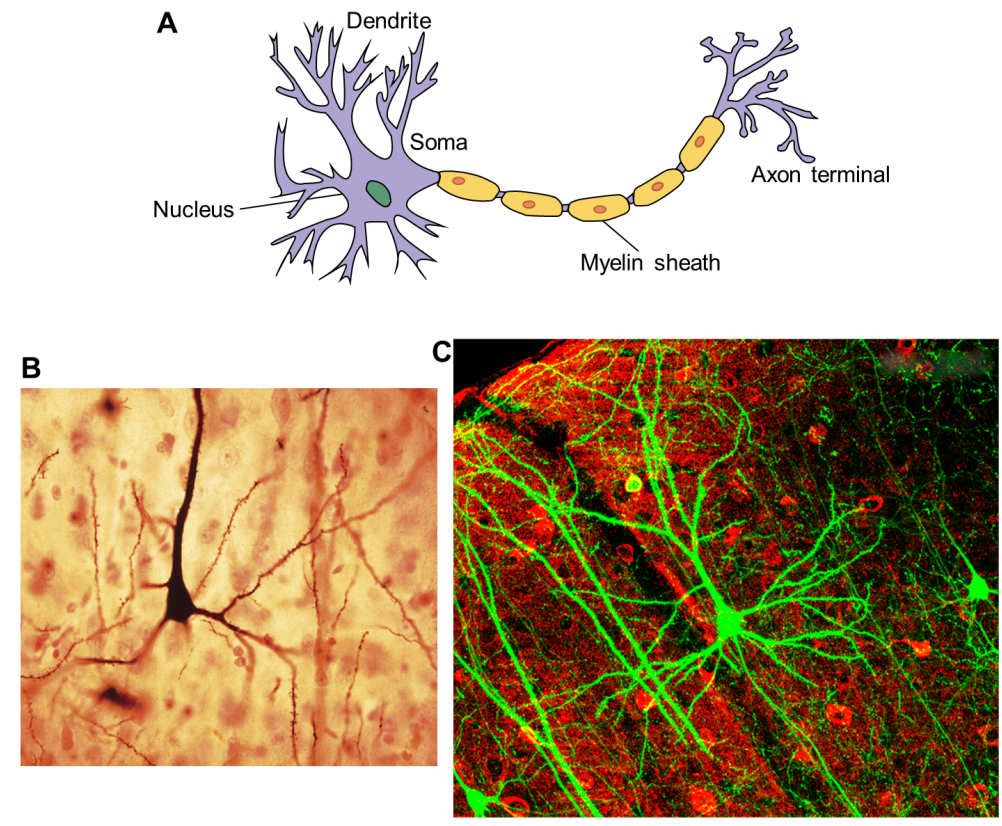

Figure 2.2: Neurons underlie the electrical signaling in the brain. A: A schematic drawing of a generic neuron. Note that the main parts of the neuron in this picture are the axon and the cell body (soma). Figure adapted from [8], with permission. B: Golgi-stained pyramidal neuron in the cerebral cortex of a cat. Figure adapted from [7], with permission. The drawing originates from the Cajal Institute in Madrid. C: Staining of neurons in the rat visual cortex using immunofluorescence techniques. Pyramidal neurons are shown as green cells, while GABA inhibitory interneurons are shown as red cells. The pyramidal neurons exhibit both apical (upwards) and basal (sideways) dendrites. The axons extend down from the soma in the lower portion of the image. This figure was adapted from [9], with permission.

Neurons are canonically comprised of four functionally unique regions (Fig. 2.2A): dendrites, the cell body (soma), the axon, and the axon terminals [1]. Dendrites are thin and spiny branches that extend outwards from the soma, and are the recipient of incoming signals from other neurons through synapses. On average, each neuron is connected through approximately 7000 synapses [10]. The soma houses the nucleus, which contains the genetic set of instructions that encode the synthesis of proteins in the neuron. Axons are long processes which conduct the action potential from the soma to a synapse. Some neurons sheath the axons in the protein myelin, which acts as an insu- 


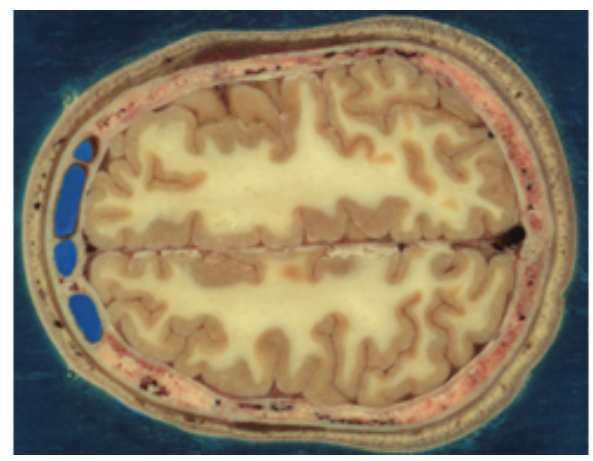

Figure 2.3: Axial view of the human brain. The characteristic ridges (gyri) and fissures (sulci) are seen on the surface of the cortex. The cortex contains a larger amount of neuronal cell bodies and therefore appears gray. The deeper white regions contain larger amounts of myelinated axons. Image reprinted from the Visible Human Project [11]).

lator to promote the electrical conduction of vast segments of the axon [1]. The axon terminal is found at the tip of the axon, which constitutes the presynaptic half of the synapse. The other half of the synapse is the post-synaptic dendrites of the receiving neuron. As no direct structural connection exists between the neurons, the electrical signal must be converted to an intermediary messenger, a neurotransmitter, which is released into the synaptic cleft. The neurotransmitter binds to specific receptors on the post-synaptic neuron, which when opened, induce an action potential, thereby relaying the 'message' further on.

Numerous different neurotransmitters are used in the central nervous system. Neurotransmitters are often classified as either excitatory or inhibitory, but the effect is not intrinsic to the neurotransmitter but instead depends on the post-synaptic expression of receptors. Nevertheless, glutamate and $\gamma$-aminobutyric acid (GABA) are the two most prevalent excitatory and inhibitory neurotransmitters used in the nervous system, respectively [1]. Excitatory neurotransmitters evoke small positive change in the post-synaptic membrane potential, whereas inhibitory neurotransmitters evoke a counteracting effect. The release of neurotransmitters is dependent on the type of neuron activated. Excitatory neurons such as a pyramidal neuron (Fig. 2.2B$\mathrm{C}$, Fig. 2.6B) release glutamate when depolarized. Other types of neurons, such as GABAergic interneurons (Fig. 2.2C, Fig. 2.6B), are predominantly inhibitory and release GABA when stimulated.

\section{Cortical arrangement of neurons}

The large scale organization of the nervous system shares a similar structure in all mammals. The tissue which the brain consists of is commonly divided 
into two parts: gray matter and white matter (Fig. 2.3). Gray matter contains a high density of neuronal cell bodies, while white matter contains mainly myelinated axons. As seen, the cortex (outer layer) of the brain is predominantly made up of gray matter, and has a characteristic folding pattern of grooves (denoted sulci) and ridges (denoted gyri). This clever structural pattern increases the surface area of the cortex (and thereby the number of neurons), which has allowed for higher-order cognitive abilities to develop. The

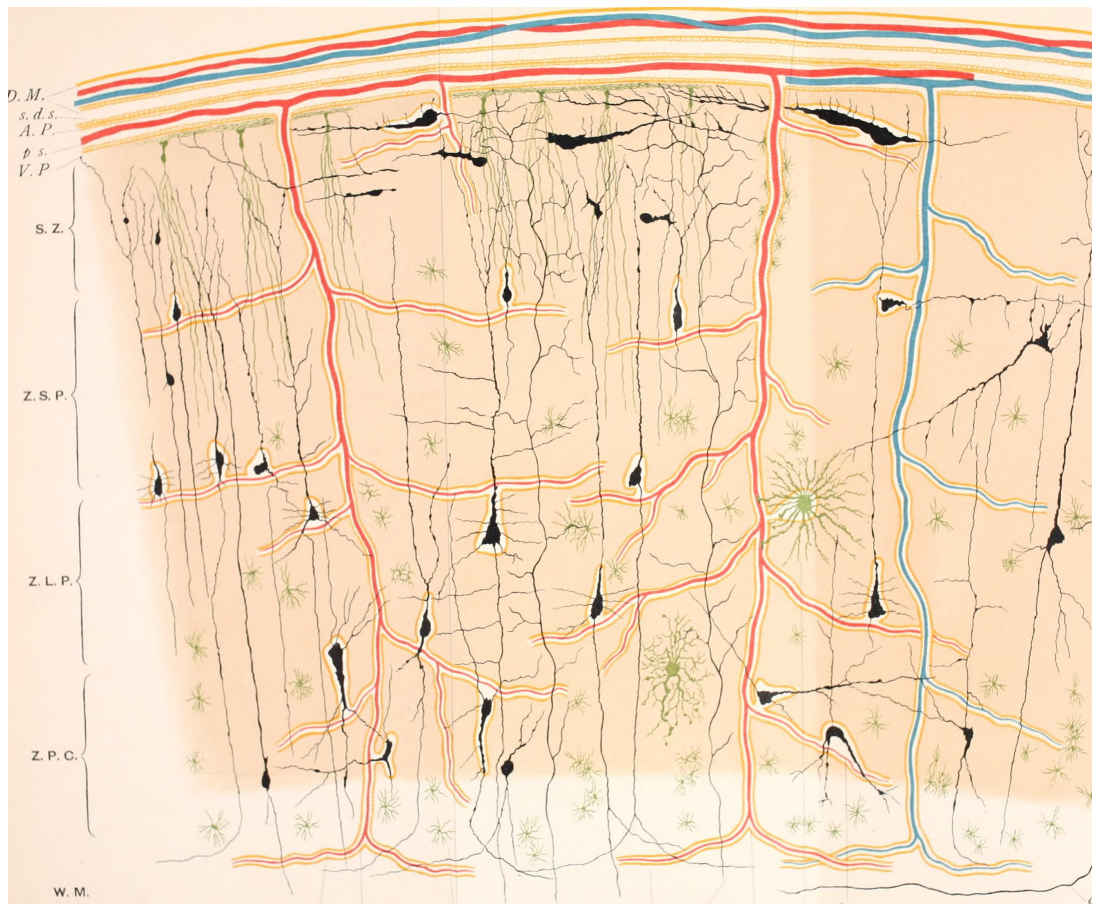

Figure 2.4: The column-like structure of the cortex. Each layer (see left side) possess a distinct selection of neuronal types and connections which spans both between and outside of the layers. The supply of blood is depicted with red (arteries and arterioles) and blue (veins and venules) blood vessels which penetrate the cortical column. White matter can be seen at the bottom of the column, with axons spanning both upwards and downwards directions. Image adapted from [12], with permission.

cortex has a column-like structure consisting of up to six (I-VI) distinct layers of neurons. These columns are aligned perpendicular to the surface (Fig. 2.4). The type of neurons and the intra-layer or extra-layer connections are specific to that layer and area, and different layers receive input from other cortical areas (see bottom of the figuring for traces of axons in white matter). Nevertheless, excitatory pyramidal neurons are commonly expressed in layer 5 (see Z.L.P in Fig. 2.4, dark pyramidal shapes), and inhibitory (GABAergic) interneurons in layer II/III (see irregularly shaped spiny neurons in Fig. 2.4). Note the vascular penetration of arteries and arterioles (red vessels), 
and correspondingly venules and veins (blue vessels), throughout the cortical column. The seminal work by Vernon Mountcastle (1918-2015) in the cat somatosensory cortex showed that neurons that respond to a specific stimuli often occupy the same layer in a cortical column. This insight spawned the idea that these cortical columns constitute the "elementary unit of organization" in the brain [13].

Finally, to transition from the structure of cortical columns to more largescale cortical regions, the neurologist Korbinian Brodmann (1868-1918) used the cytoarchitectonic differences of the cortical columns to divide the brain into 52 distinct areas or regions [14]. Brodmann postulated that areas with different structural composition correspond to different functions of the brain [15], which holds true for some of the Brodmann areas today [15]. These areas are depicted in Figure 2.5.

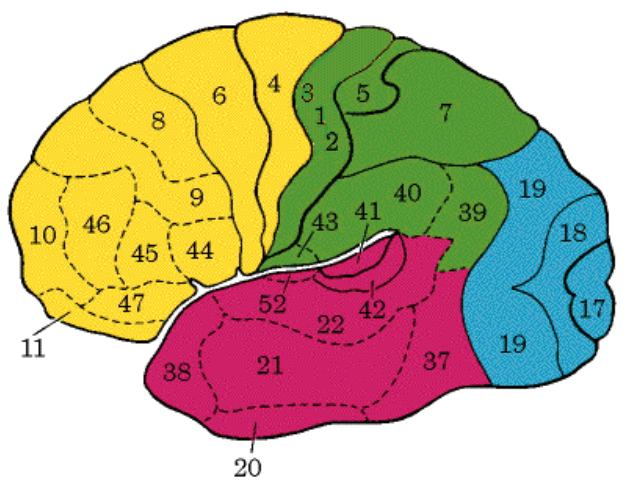

Figure 2.5: Brodmann's map of cytoarchitectonics. The numbers correspond to brain regions that possess a distinct cytoarchitecture. Image adapted from [16], with permission.

To summarize, we begun this section with the function of action potentials; moving up through the morphology and function of neurons; their vertical alignment into cortical layers and columns, and finally, the large scale division of specialized cortical areas with the help of the Brodmann classification. Throughout this brief journey through the history and different spatial scales of neuroscience, we have acquired a small insight into the complexity of the mammal brain. A natural question arises, how does the brain maintain its normal function with all of this complexity? 


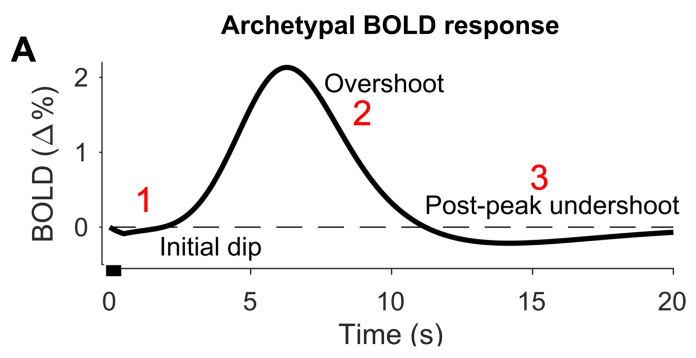

B

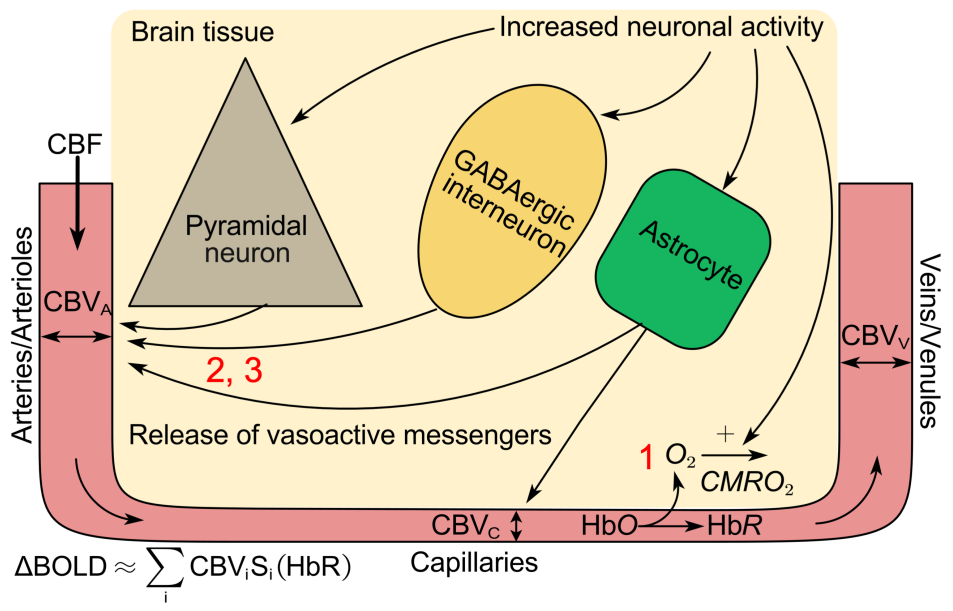

Figure 2.6: Brief overview of functional hyperemia and the underlying biological system. A: The archetypal BOLD response exhibits three main characteristics to short stimuli: 1) a debated initial dip, 2) an over-compensatory response ('overshoot'), and finally, 3) a post-peak undershoot. B: The intracellular signaling basis of the NVC. Increased neuronal activity triggers increased cerebral metabolic rate of oxygen $\left(\mathrm{CMRO}_{2}\right)$, which can sometimes be observed as an initial dip (1). Simultaneously, neurons and astrocytes release vasoactive messengers upon stimulation, evoking changes in cerebral blood flow (CBF) and volume (CBV), which underlie the overshoot and post-peak undershoot phases of the response $(2,3)$. BOLD $=$ Blood Oxygen Level-Dependent

\subsection{A short introduction to the neurovascular coupling}

The brain constitutes $\approx 2 \%$ of the total body weight of an average adult human [17]. Despite this small weight proportion, the brain consumes $\approx 20 \%$ of an adult's total energy [17]. To satisfy this large demand for energy, the brain requires a non-stop supply of metabolic substrates such as glucose and oxygen [18]. Such metabolic substrates are delivered through blood circulation, and can be transported over from blood to cerebral tissue in the capillaries through diffusion or receptor-mediated transport. This connection between brain activity and the supply of blood was first discovered by Mosso in 1881 
[19], and later by Roy and Sherrington in 1890 [20]. These two studies unraveled a temporal and spatial connection between alterations in brain activity and local hemodynamic changes - a neurovascular coupling (NVC). We now know that the NVC describes how the communication between neural cells and the vasculature actively regulates cerebral blood flow (CBF), cerebral blood volume (CBV), and cerebral metabolic rate of oxygen $\left(\mathrm{CMRO}_{2}\right)$. More than a century later, a detailed view of the communication has evolved. The NVC is initiated by the synaptic activity of neurons, brain activity, which triggers increased metabolism of oxygen and glucose, as well as intracellular signaling cascades that culminate in the release of many different vasoactive molecules (see reviews in: [21-26]). These vasoactive messengers originate from different types of neurons, including pyramidal neurons, GABAergic interneurons, or other neural cells such as astrocytes (Fig. 2.6B).

The actions of these different cell types underlie a common response pattern that is often observed for local increases in brain activity. This response pattern consists of three main characteristics: 1) an initial rapid increase of $\mathrm{CMRO}_{2}$ (Fig. 2.6A-B, see 1), followed by 2) large hemodynamic changes with a characteristic oversupply of blood Fig. 2.6A-B, see 2). The oversupply of blood flow compensates fully for the increased metabolism, leading to an increased blood and tissue oxygenation, and is referred to as functional hyperemia. Finally, a post-peak undershoot can sometimes be observed, where the blood oxygenation and flow decrease below basal for a short time as it returns to baseline values (Fig. 2.6A-B, see 3). Functional hyperemia forms the basis of functional neuroimaging techniques, which exploit this increased blood oxygenation as a biomarker for brain activity [27]. These mostly non-invasive techniques include the widely used functional magnetic resonance imaging (fMRI) [28, 29] or near-infrared spectroscopy (NIRS) [30-32], which have been used to unravel a plethora of insights regarding brain function and cognition during the last 30 years [33]. In particular, fMRI captures a Blood Oxygen Level-Dependent (BOLD) signal, such as the response depicted in Figure 2.6A. Nevertheless, due to the indirect nature of this measure of brain activity, it is essential to have a complete understanding of the molecular and cellular chain of events underlying the NVC in order to fully interpret functional neuroimaging data. While this detailed chain of events still remains elusive, a broad picture of the essential mechanisms has slowly emerged. However, the analysis and interpretation of functional neuroimaging data is conventionally done in a statistical way that ignores the current understanding of the underlying biological mechanisms.

This statistical way is typically based on a correlational analysis between the observed BOLD-fMRI data (Fig. 2.7A) and a template of what the response should look like, using the general linear model (GLM) and its associated hemodynamic response function (HRF) (Fig. 2.7B) [34, 35]. This 


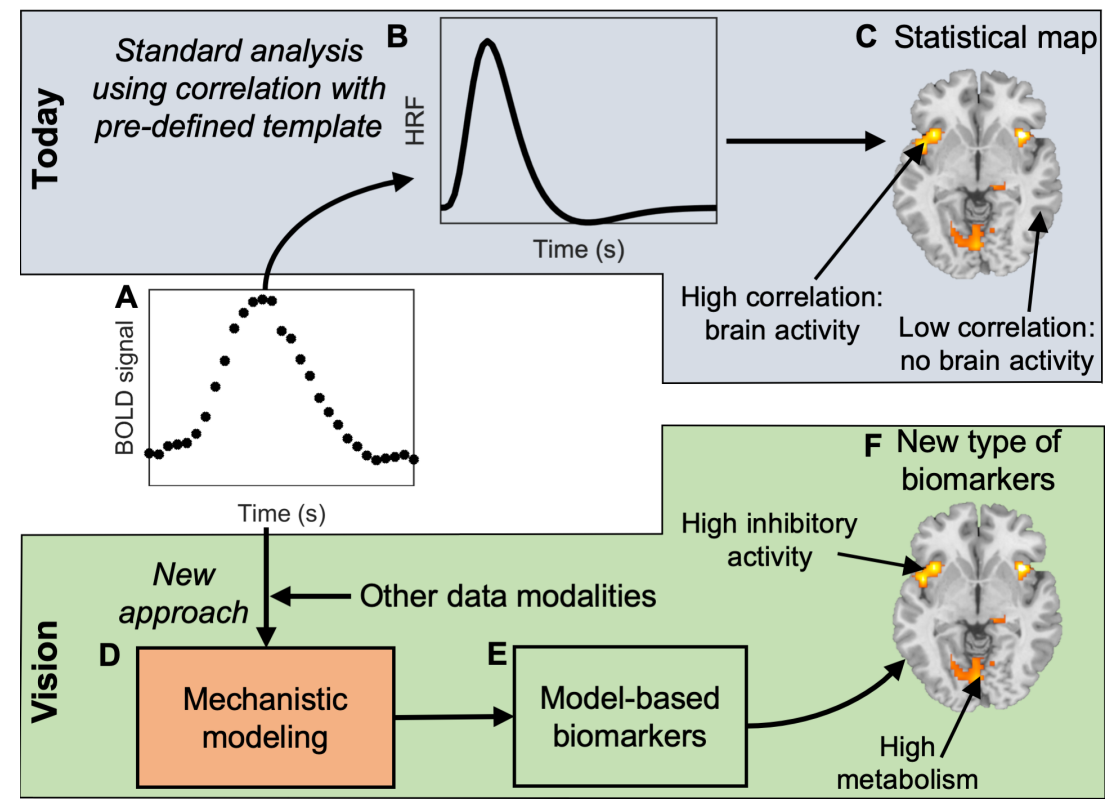

Figure 2.7: Analysis of fMRI: today and a vision for the future. BOLD-fMRI data (A) are analyzed using a correlation analysis with a standard defined template, a hemodynamic response function (HRF) (B). This analysis produces statistical maps that depict areas with high or low correlations which are used to infer brain activity (C). In contrast, a model based on the underlying biochemical and physiological mechanisms could be utilized (D), which also could incorporate more data into the analysis. Such an approach would give model-based biomarkers (E-F), for instance, increased metabolism or increased activation of different subsets of neurons (F).

correlation analysis equates a high correlation between the measured BOLDfMRI signal and the HRF as brain activity, and a low correlation as no brain activity, for the measured brain area (Fig. 2.7C). In contrast, the use of mathematical models which incorporate more information about the underlying system (Fig. 2.7D) can in principle include other measured observables in the analysis, such as CBF or CBV. Furthermore, such a model-based approach could also produce new model-based biomarkers (Fig. 2.7E). These biomarkers could potentially be more nuanced than the current correlation approach and identify things such as increased metabolism or increased activation of different subsets of neurons. (Fig. 2.7F). However, no useful mathematical model exists that could make this vision a reality. More specifically, models are needed that include biochemical mechanisms of the NVC. Such models, which are built upon biochemical or biological mechanisms, could be developed using tools and methods from the field of Systems Biology. 


\subsection{Systems Biology}

An new interdisciplinary research field - Systems Biology - emerged in the early 2000s [36-39]. Systems Biology aims to provide a systems-level wide understanding of biological systems, and to capture how underlying system properties arise only when combining individual pieces from the same puzzle or system. This stands in contrast to the classical reductionist approach, where each component (or puzzle piece) is examined in isolation, a common approach in in vitro molecular biology [39]. Systems Biology rests on two core pillars: 1) acquisition of experimental data from the intact biological system, and 2) the use of mathematical models to analyze that data to generate new insights regarding the system.

The first concept, acquisition of experimental data from the system, is based on the fact that a systems-level understanding can only be obtained if enough diverse experimental data can be captured from the intact system. The second concept, mathematical modeling, is useful for analyzing complex data sets as it provides a framework for quantitatively testing hypotheses about the inner workings of the system, and in turn, how the captured experimental data was generated. As Systems Biology aims to capture all of the pieces of a given system, which are often complex and interconnected, the generated models and explanations are often also complex [36, 39]. This is typically the case for mechanistic modeling, where the components of the model, as well as the system properties that arise from the inter-connected components, can be understood in biological terms. Nevertheless, these types of mechanistic models have until recently rarely been applied to biological systems, due to previous experimental limitations of how much data that can be obtained from the system.

\section{Models of the NVC}

In the context of NVC, a variety of modeling approaches have been used for analysis of the NVC (see Table 1, first column). Early work by Friston et al. [41] and Buxton et al. [40, 52], and later by Havlichek et al. [42] introduced variants of the 'Balloon' model, a quasi-phenomenological model describing the interplay between $\mathrm{CBF}, \mathrm{CBV}$, and $\mathrm{CMRO}_{2}$. While these models have been of great importance for the interpretation and development of fMRI, these models have three main limitations. First, these models are not based on the cellular and molecular mechanisms underpinning the NVC, and instead utilize phenomenological or bio-mechanical descriptions to connect neuronal activity to hemodynamic changes (see Table 2.1, second column). Second, these models cannot account for recent experimental data which show that arteriolar dilation is the main driver of the hemodynamic responses (see [53] and references therein), instead only accounting for a venous vas- 
Table 2.1: Comparison of different published models describing the neurovascular coupling with regards to different aspects. The notations used in the table is, 'Yes' if the model features the stated aspect; 'Partly' if the model has a description that is not fully satisfying, and 'No' if there is no satisfying description of the aspect. As seen, no model currently exists that can describe all aspects. Table adapted from Paper IV, with permission.

\begin{tabular}{l|c|c|c|c} 
Models & $\begin{array}{c}\text { Intracellular } \\
\text { mechanisms }\end{array}$ & $\begin{array}{c}\text { All vascular } \\
\text { compart- } \\
\text { ments }\end{array}$ & $\begin{array}{c}\text { Blood } \\
\text { oxygenation } \\
\text { dynamics }\end{array}$ & $\begin{array}{c}\text { Full BOLD } \\
\text { description }\end{array}$ \\
\hline $\begin{array}{l}\text { Buxton et al., } \\
\text { 1998 [40, 52]; }\end{array}$ & No & No & Partly & Partly \\
$\begin{array}{l}\text { Friston et al., } \\
\text { 2000 [41]; } \\
\text { Havlicek et al., } \\
\text { 2015 [42] }\end{array}$ & & & & \\
\hline $\begin{array}{l}\text { Barrett et al., } \\
\text { 2012 [43-45] }\end{array}$ & No & Yes & Yes & No \\
\hline $\begin{array}{l}\text { Huppert et al., } \\
\text { 2007 [46-48] }\end{array}$ & No & Yes & Yes & Yes \\
\hline $\begin{array}{l}\text { Yücel et al., } \\
\text { 2009 [49]; } \\
\text { Mathias et al., } \\
\text { 2016 [50, 51] }\end{array}$ & Yes & No & Partly & Partly \\
\hline $\begin{array}{l}\text { Final work of } \\
\text { this thesis }\end{array}$ & Yes & Yes & Yes & Yes
\end{tabular}

cular compartment (as these models were originally developed to describe the mismatch between CBF and CBV; see Table 2.1, third column). Third, these models do not explicitly include blood oxygenation and saturation dynamics (Table 2.1, fourth column), which in combination with the previous limitation, leads to only a partial description of the BOLD signal (Table 2.1, last column). Others research groups have developed more advanced electrical circuit equivalent vascular models, which remedy in particular the second and third limitation, such as Huppert et al. [46-48] or Barrett et al. [43-45]. Nevertheless, mechanistic models based on the current understanding of the molecular underpinnings of the NVC are lacking. Some models exist, such as models proposed by Yücel et al. [49] and Mathias et al. [50, 51]. However, these models have rarely been trained to time-resolved experimental data, and are typically connected to the 'Balloon' model in order to simulate the hemodynamic responses. Therefore, a model based on the core pillars of Systems Biology remains to be determined. 


\subsection{Aims and scope}

The overall vision is to take the first steps towards obtaining a mathematical model built on the molecular mechanisms underpinning the NVC, which can be used to: 1) explain commonly observed NVC phenomena; 2) provide a more exhaustive interpretation of functional neuroimaging data with the potential of introducing new types of biomarkers, and 3) generate new insights into the functions of the NVC. More specifically, in this thesis, the main focus lies on applying the methods and tools used within Systems Biology to construct mathematical models built on the known mechanistic underpinnings of the NVC (Fig. 2.7D, orange box). These models are developed and investigated using in vivo experimental data from multiple different species and experimental modalities. 


\section{3}

\section{Neurovascular Coupling}

Throughout this chapter, I will introduce the reader to the seminal studies behind the discovery of the NVC; the current state-of-the-art understanding of the molecular and cellular basis of the NVC; a selection of in vivo measurement modalities used to interrogate the NVC, and finally, how the NVC is affected by commonly used anesthetics within NVC research.

\subsection{The discovery of a NVC}

As briefly mentioned above, during the late 19th century, Angelo Mosso (1846-1910) and separately the duo of Charles Roy (1854-1897) and Charles Sherrington (1857-1952), provided the first evidence of a NVC by unraveling the coupling between brain activity and energy supply. Mosso observed brain pulsations occurring in the prefrontal cortex of a human subject with a posttraumatic skull defect, using a 'cerebral pulsometer' [19, 54] (Fig. 3.1). These pulsations held the same pace as the subject's heartbeat, but the amplitude of the brain pulsations increased independent of the heartbeat when the subject performed different cognitive tasks, such as reciting 'Ave Maria' (Fig. 3.1 top, the onset of the cognitive task is marked by the black arrow) [19]. These findings were followed up by the seminal work of Sherrington and Roy in 1890 [20], who employed a more direct approach which revealed that stimulation of sensory nerves in animals produced an increase in cerebral blood pressure and volume. Furthermore, the duo also observed that cerebral metabolites released during brain asphyxia (hypoxia/hypercapnia) dilated the cerebral vasculature in a compensatory manner [20]. With these key deductions and other findings, they arrived at the following conclusions:

"We conclude then, that the chemical products of cerebral metabolism contained in the lymph which bathes the walls of the arterioles of the brain can cause variations of the calibre of the cerebral vessels: that in this reaction 


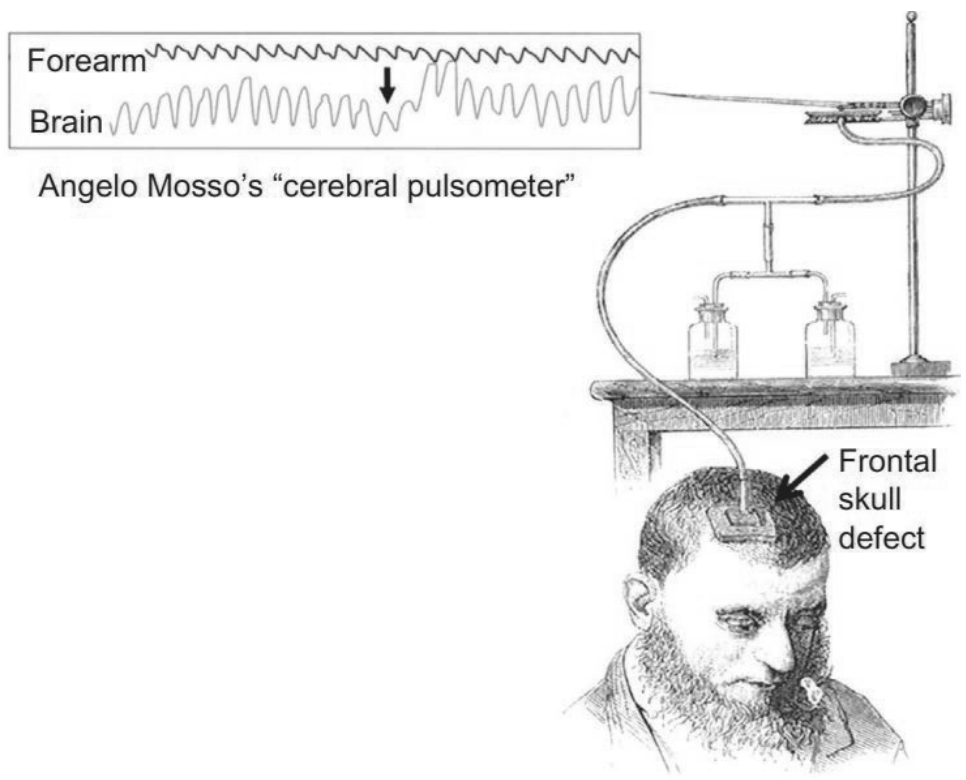

Figure 3.1: Cerebral pulsations in the prefrontal cortex of a thin skulled human with simultaneously measured heartbeats at the forearm. The subject was task with reciting 'Ave Maria', with the onset indicated by the black arrow. Image taken from [54] and [55], with permission.

the brain possesses an intrinsic mechanism by which its vascular supply can be varied locally in correspondence with local variations of functional activity" [20].

In other words, Roy and Sherrington proposed the mechanism of metabolic feedback, connecting cerebral metabolism with changes in localized CBF.

\section{The proposal of the metabolic feedback hypothesis}

Feedback mechanisms are a common and central component in the regulation of many physiological and biological systems. The metabolic feedback hypothesis of CBF regulation, proposed by Roy and Sherrington [20], states that signals which increase localized CBF are generated as a result of reduced supply of metabolic substrates and/or an accumulation of metabolic waste products (see review in [56]). An intuitive initiator for the neurovascular response could be for instance, a rapid reduction in the local concentration of oxygen and/or glucose, i.e. a negative feedback loop. Previous studies have shown that brain metabolism is more sensitive to reductions in oxygen concentration than glucose (see [57] and references therein). Indeed, earlier studies have shown that a decrease in cerebral tissue oxygen levels is strongly correlated with increased neuronal activity [58-60], and that changes in CBF 
correlate with oxygen usage [61] in vivo. In addition, an in vitro study of rat brain slices demonstrated that oxygen levels in tissue determine the polarity of hemodynamic responses induced by activation of astrocytes [62]. However, correlation does not equal causality, and further available evidence suggests that the lack of oxygen or glucose does not directly increase the CBF ([63-65], also see [66] and references therein). In addition, the hemodynamic responses are not affected by hyperoxia for both normobaric [65] and hyperbaric [67] experimental conditions. These studies together suggest that neither oxygen or glucose directly evokes the transient CBF responses observed upon increased neural activity.

Another potential mechanism is that the waste products of cerebral metabolism such as $\mathrm{CO}_{2}, \mathrm{H}^{+}$, or lactate could evoke hemodynamic changes. Some studies have shown that astrocytes are capable of sensing experimental manipulations of $\mathrm{CO}_{2}$ or $\mathrm{H}^{+}$, and respond to such manipulations with increased intracellular $\mathrm{Ca}^{2+}$ and release of adenosine triphosphate (ATP), which leads to the release of vasoactive signaling molecules $[68,69]$. Nevertheless, no direct effect of $\mathrm{CO}_{2}$ and $\mathrm{H}^{+}$can account for the transient changes in $\mathrm{CBF}$ evoked by stimulation [70, 71]. Finally, lactate is also released upon local increases in neural activity, and has been shown to positively modulate the hemodynamic response, most likely by up-regulating nitric oxide production (see review in [72] and references therein).

To summarize, these studies together suggest no direct effect of the cerebral metabolites on functional hyperemia, but instead, a subset of the metabolites can indirectly modulate and shape the response through effects on the production and release of intracellular vasoactive messengers. Therefore, the question arises: how are these vasoactive messengers released?

\subsection{Feed-forward hypothesis governing the NVC}

An alternative hypothesis to the metabolic feedback hypothesis is the idea of a feed-forward mechanism (see reviews in [22, 24, 26, 73]). A feed-forward mechanism describes how active neurons and astrocytes release vasoactive messengers or signaling molecules that evoke vasodilation or vasoconstriction, through direct or indirect action on the vasculature. The key difference between a feed-forward and a feedback regulated mechanism is that a feedforward mechanism acts pro-actively, while a feedback mechanism acts reactively. Furthermore, in the context of the NVC, the signaling molecules which underpins the hemodynamic response are separate from those involved in cerebral metabolism. This hypothesis has gained traction with the discovery of many different feed-forward signaling pathways that influence the NVC (see Fig. 3.2). These discoveries have unraveled a basic chain of events, which includes multiple different cell types, signaling pathways, and 
finally, secretion of different vasoactive messengers or molecules which act on their associated vascular targets.

This basic chain of events begins with the release of neurotransmitters from pre-synaptic neurons, which bind to specific ion channel coupled receptors expressed on the post-synaptic neurons. The main excitatory neurotransmitter, glutamate, bind to N-methyl-D-aspartate (NMDA) and $\alpha$-amino-3-hydroxy-5-methyl-4-isoxazolepropionic acid (AMPA) receptors. Activation of these receptors opens their ion-conducting pores, causing an influx of $\mathrm{Na}^{+}$and $\mathrm{Ca}^{2+}$ ions, and subsequently, depolarization of the neuron. Depolarization opens voltage-gated calcium channels, causing an additional influx of $\mathrm{Ca}^{2+}$ ions. In contrast, the primary inhibitory neurotransmitter GABA acts to prevent depolarization of neurons. GABA binds, for instance, to the ion channel-coupled $\mathrm{GABA}_{\alpha}$ receptor, which opens $\mathrm{Cl}^{-}$ ion-conducting pores, causing an influx of $\mathrm{Cl}^{-}$ions which counterbalance any depolarizing efforts. Excitatory neurons such as pyramidal neurons are glutamatergic, meaning that glutamate is released upon depolarization, and acts on astrocytes and other neurons. Inhibitory neurons, such as GABAergic interneurons release GABA upon depolarization and act on neurons or other neural cells. In total, these neuronal interactions form a canonical neuronal circuit, where pyramidal neurons excite the neighboring neural cells, and GABAergic interneurons regulate the activity of surrounding neurons. Furthermore, depolarization causes an influx $\mathrm{Ca}^{2+}$ ions, which in turn triggers cell-specific signaling pathways.

\section{Pyramidal neurons}

The rise in intracellular $\mathrm{Ca}^{2+}$ levels in pyramidal neurons (Fig. 3.2, gray triangle) activates phospholipases which cleave membrane phospholipids through a series of enzymatic steps into arachidonic acid (AA) [24, 26, 74]. $\mathrm{AA}$ is metabolized into prostaglandin $\mathrm{E}_{2}\left(\mathrm{PGE}_{2}\right)$ through a cyclooxygenase (COX)-2 rate-limiting conversion [75-78]. Finally, $\mathrm{PGE}_{2}$ promotes vasodilation through activation of $\mathrm{PGE}_{2}$ receptors $\mathrm{EP} 2$ and $\mathrm{EP} 4$ located on vascular smooth muscle (VSM) cells or pericytes, which up-regulates the synthesis of cyclic adenosine monophosphate (cAMP) (Fig. 3.2) [76, 79].

\section{GABAergic interneurons}

The rise in intracellular $\mathrm{Ca}^{2+}$ levels in GABAergic interneurons evoke the release of different vasoactive molecules or peptides (Fig. 3.2, yellow cell body). For instance, nitric oxide (NO) evokes vasodilation of arterioles, in both in vitro cortical slices and in vivo [80-83]. NO is released by specific interneurons which express the enzyme neuronal nitric oxide synthase (nNOS) [84], which is modulated by $\mathrm{Ca}^{2+}$. A recent systematic review revealed that inhibition of nNOS had the largest effect on the hemodynamic response for in vivo 


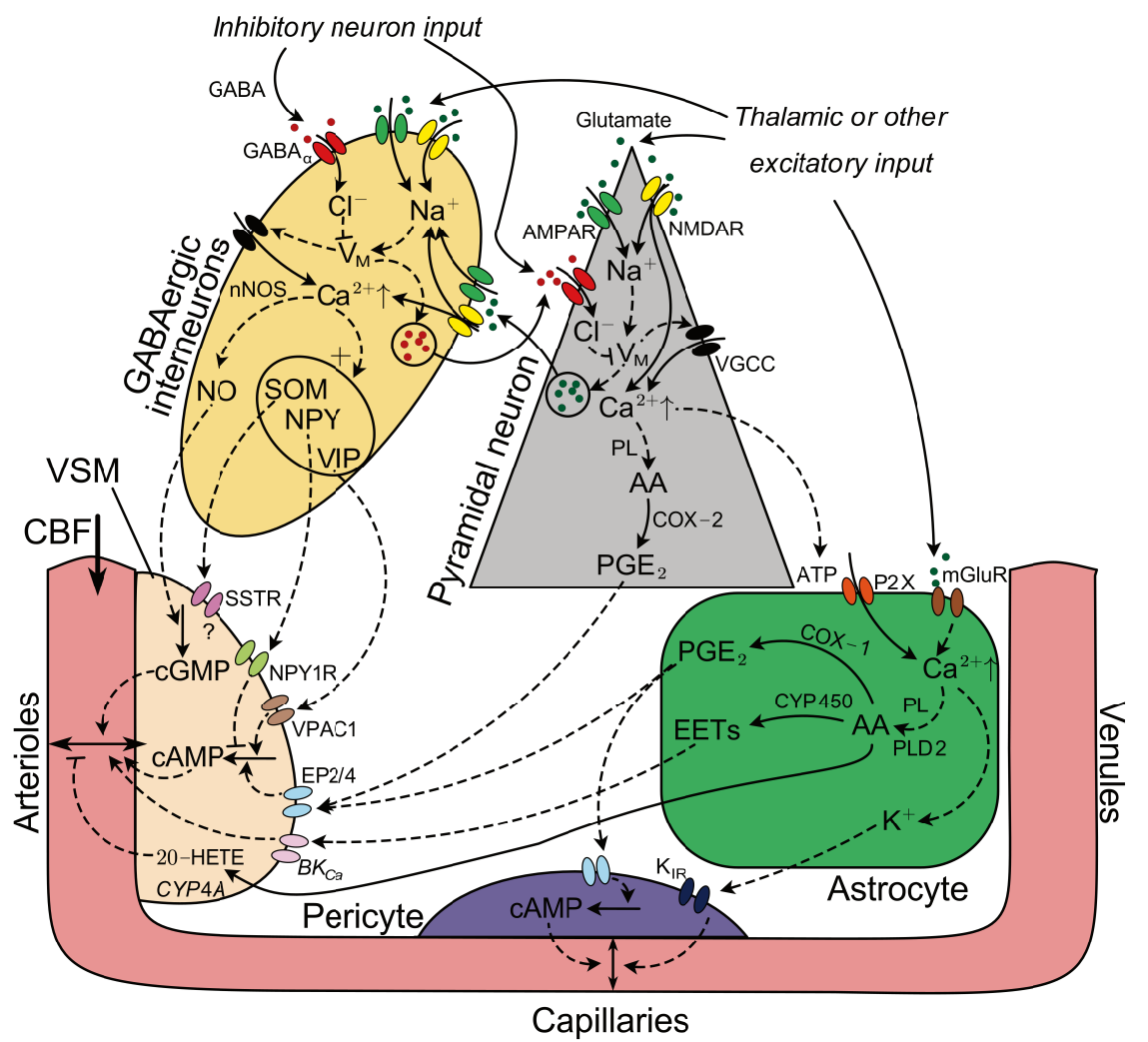

Figure 3.2: Simplified schematic illustration of the cellular pathways underlying the release of vasoactive messengers in the $\mathbf{N V C}$. Neuronal signaling affects GABAergic interneurons (yellow oval) and pyramidal neurons (gray triangle), inducing fluctuations in membrane potential $\left(\mathrm{V}_{\mathrm{M}}\right)$ through activation of $\alpha$-amino-3-hydroxy-5-methyl-4-isoxazolepropionic acid (AMPA), N-methyl-D-aspartate (NMDA) or $\gamma$-aminobutyric acid (GABA $\alpha$ ) receptors. Depolarization induces an influx of $\mathrm{Ca}^{2+}$ ions. $\mathrm{Ca}^{2+}$ acts to facilitate various vasoactive signaling pathways in the different cells. In GABAergic interneurons, $\mathrm{Ca}^{2+}$ promotes the production and subsequent release of nitric oxide (NO) through up-regulation of neuronal nitric oxide synthase (nNOS), as well as facilitating the release of different neuropeptides such as neuropeptide Y (NPY), vasoactive intestinal peptide (VIP), and somatostatin (SOM). In pyramidal neurons, $\mathrm{Ca}^{2+}$ promotes arachidonic acid (AA) synthesis via phospholipases (PL), which in turn is processed into prostaglandin E2 ( $\mathrm{PGE}_{2}$ ) via cyclooxygenase-2 (COX)-2. Glutamatergic signaling also affects astrocytes (green rectangle), inducing a $\mathrm{Ca}^{2+}$ influx through the ATP-sensitive P2X receptor or by activation of the metabotropic glutamate receptor (mGluR). In astrocytes, AA is synthesized via phospholipase D2 (PLD2), and subsequently metabolized into three different vasoactive molecules: epoxyeicosatrienoic acid (EET) via cytochrome P450 (CYP) epoxygenase, 20-Hydroxyeicosatetraenoic acid (20-HETE) via CYP4A, and finally, $\mathrm{PGE}_{2}$ via $\mathrm{COX}-1$. These vasoactive messengers act indirectly by modulating the production of cyclic adenosine monophosphate (cAMP) or cyclic guanosine monophosphate (cGMP) or directly on $\mathrm{K}^{+}$channels, through activation of messenger specific ion channels or receptors located on the cell membrane of vascular smooth muscle (VSM) cells or pericytes. Finally, the activation of astrocytes triggers the release of $\mathrm{K}^{+}$ions. $\mathrm{K}^{+}$act on inwards rectifying $\mathrm{K}$-channels $\left(\mathrm{K}_{\mathrm{IR}}^{+}\right)$located on pericytes, inducing relaxation. Together, all of these different vasoactive messengers act on the vasculature, evoking 
changes in cerebral blood flow (CBF). Neuronally-derived messengers act primarily on the arterioles, while astrocytic-derived messengers exert their effect on both arterioles and capillaries. Somatostatin receptor (SSTR); Neuropeptide receptor Y1 (NPY1R); VIP/PACAP receptor type 1 (VPAC1); large-conductance calcium-activated $\mathrm{K}^{+}$channels $\left(\mathrm{BK}_{\mathrm{Ca}}\right)$; Prostaglandin $\mathrm{E} 2$ receptor (EP).

studies $(n=11)$ [85]. NO acts by increasing the synthesis of cyclic guanosine monophosphate (cGMP) in VSM cells [85].

GABAergic interneurons also express different neuropeptides which exert vasoactive effects both in vitro and in vivo [23, 77, 81, 86]. These neuropeptides can induce both vasodilation through vasointestinal peptide (VIP) [23, 81,87 , or vasoconstriction through both neuropeptide Y (NPY) [81, 88-90] or somatostatin (SOM) $[81,91,92]$. VIP acts on the VIP receptor type 1 which up-regulates the production of cyclic adenosine monophosphate (cAMP) in VSM cells [23]. NPY binds to the NPY receptor Y1 (NPY1R), a $\mathrm{G}_{\alpha i}$-protein coupled receptor which is expressed on the membrane of VSM cells [93, 94]. Activation of NPY1R inhibits the synthesis of cAMP, causing vessel constriction [95]. Finally, SOM acts possibly on somatostatin receptors (SSTR) to induce vasoconstriction [81].

To unravel the contributions of excitatory and GABAergic neurons to the evoked hemodynamic responses, optogenetic (see Section 3.3 for a brief overview) stimulation and/or different pharmacological manipulations can be used. One such study showed that the ratio between increased metabolic demand and the blood flow/volume change differs between selective activation of pyramidal neurons and GABAergic interneurons [96]. Pyramidal neurons have a much larger ratio, implying a larger metabolic cost associated with activation. In contrast, activation of GABAergic interneurons carries a low metabolic cost, and evokes a larger flow and volume increase as compared to pyramidal neurons [96].

\section{Astrocytes}

Astrocytes have end-feet ideally placed around smooth muscle cells on arterioles or pericytes, making them a potential mediator of the NVC (Fig. 3.2, green rectangle). Furthermore, astrocytes are known to regulate the basal tone of arteries [97-100]. The release of neurotransmitters such as glutamate and GABA can influence astrocytes directly. Glutamate binds to metabotropic glutamate receptors $\mathrm{mGluR}_{1}$ and $\mathrm{mGluR}_{5}$, resulting in a inositol-1,4,5-trisphosphate receptor (IP3 R) mediated release of $\mathrm{Ca}^{2+}$ from internal storage pools (see [101] and references therein). The rise in intracellular $\mathrm{Ca}^{2+}$ levels in astrocytes activates phospholipase D2, which cleaves membrane phospholipids into AA [80, 102]. AA is metabolized into different vasoactive substances such as $\mathrm{PGE}_{2}$ via $\mathrm{COX}-1$, epoxyeicosatrienoic 
acids (EETs) via cytochrome $\mathrm{P} 450$ (CYP), or 20-hydroxyeicosatetraenoic acid (20-HETE) via CYP4A (see [26] and references therein). EETs act on large-conductance calcium activated $\mathrm{K}^{+}$channels $\left(\mathrm{BK}_{\mathrm{Ca}}\right)$, inducing relaxation through hyper-polarization. [24, 102-106]. In contrast, 20-HETE acts to contract VSM cells by inhibiting $\mathrm{BK}_{C a}$ channels $[107,108]$. Nevertheless, despite these signaling pathways, the astrocytes' role in evoking hemodynamic responses as a naturally occurring phenomenon is unclear, as adult human and mouse astrocytes lack expression of mGluR $_{1}$ and mGluR $_{5}$ [109]. In addition, mice that lack the expression of $\mathrm{IP}_{3} \mathrm{R}$ through genetic knockout still respond robustly to stimulation in vivo, indicating that astrocytes have little contribution to evoked hemodynamic changes through this pathway [110]. Nevertheless, a recently discovered mechanism describes how ATP that is released from post-synaptic neurons triggers an influx of $\mathrm{Ca}^{2+}$ ions into astrocytes through the ATP receptor $\mathrm{P}_{2} \mathrm{X}_{1}$, provoking hemodynamic responses both in vitro and in vivo [80] (Fig. 3.2).

Finally, another vasoactive pathway involves inward-rectifier $\mathrm{K}_{\mathrm{IR}}$ channels located on pericytes and endothelial cells which surround capillaries [111]. $\mathrm{K}_{\mathrm{IR}}$ open upon extracellular increases of $\mathrm{K}^{+}$as a result of neural activity [111]. The opening of these channels induces hyper-polarization, promoting relaxation of pericytes and vasodilation of capillaries (Fig. 3.2).

In summary, many different vasoactive pathways exist that evoke both vasodilation or vasoconstriction in vitro and in vivo, and these pathways originate from multiple different neural cell types. The vasoactive messengers act primarily on the arterioles or arteries, but some have been shown to directly affect capillaries (such as in [80] or [111]). In contrast, venules and veins act strictly in a passive role. The currently available evidence points to that inhibitory neurons are the primary driver behind the observed hemodynamic responses of the NVC $[53,92,96,112,113]$. Nevertheless, to study the effect of these pathways in an intact physiological system, researchers have to rely on different in vivo measuring techniques.

\subsection{In vivo measurement techniques for capturing NVC responses}

The investigation of brain function and the NVC is reliant on the availability of experimental techniques capable of observing and measuring the ongoing biological and physiological processes in the brain. This section aims to provide a brief overview of some of the imaging modalities that have generated the experimental data used in this thesis. Nevertheless, this section is not a complete list of all the available experimental techniques. 


\section{The capture of hemodynamic responses using magnetic resonance imaging}

This section provides a brief introduction to Magnetic Resonance Imaging (MRI) in the context of NVC research. For an extended description of the principles of MRI, I refer the reader to [114-116].

\section{The basis of functional magnetic resonance imaging}

MRI revolves around the phenomenon of nuclear magnetic resonance, where the use of a strong magnetic field captures information regarding the nuclear magnetic spin. The MRI signal arises from the nuclei of hydrogen atoms $\left(\mathrm{H}^{+}\right)$, which exhibit a magnetic moment (spin). If the hydrogen nuclei are affected by an external magnetic field $\left(\mathrm{B}_{0}\right)$, the spins will tend to be aligned with the direction of the field, attaining a lower energy conformation. The nuclei will precess around the direction of the applied magnetic field, resulting in a net magnetic vector in this direction. The frequency of this precession as well as the quota of nuclei in the lower energy conformation (i.e., the size of the magnetic vector) is proportional to the strength of $\mathrm{B}_{0}$. Applying a radio frequency $(\mathrm{RF})$ pulse at the same frequency as the precession frequency (the Larmor frequency), a proportion of the nuclei will shift to the high energy conformation, tilting the mean magnetic vector slightly such that it will start to precess around the applied magnetic field vector. The tilted magnetic vector will gradually return to its equilibrium, aligning once more with the direction of the applied field through the process of relaxation, emitting electromagnetic signals that generate current that can be measured in the receiver coils. This process of transmitting RF pulses and measuring the acquired current or signal in the coils is programmed in an MR pulse sequence (a series of RF pulses). Relaxation is measured as the time it takes to regain $63 \%$ of the equilibrium magnetization after an RF pulse. This is commonly referred to as the $R_{1}$ relaxation rate, which is a tissue-specific property. After excitation by an $\mathrm{RF}$ pulse, the nuclei will spin in phase coherence, which results in a maximal transverse magnetization. This transverse magnetization will decay due to spin-spin interactions or inhomogeneities in the magnetic field. The rate of the decay is referred to as the $R_{2}$ relaxation rate if no inhomogeneities are present, and $R_{2}^{*}$ with inhomogeneities in the magnetic field.

A key revelation was found by Pauling and Coryell in 1936 [119]. They observed that hemoglobin exhibits different magnetic properties in the oxygenated state compared to the deoxygenated, with deoxyhemoglobin being paramagnetic, introducing local inhomogeneities in the magnetic field. Almost 50 years later, Thulborn et al. [120] showed that this oxygenationdependent distortion of local magnetic susceptibility could be observed in blood using MRI (as it influences $R_{2}^{*}$ ). Finally, Ogawa et al. [28] extended this technique to be capable of producing functional images of the brain 


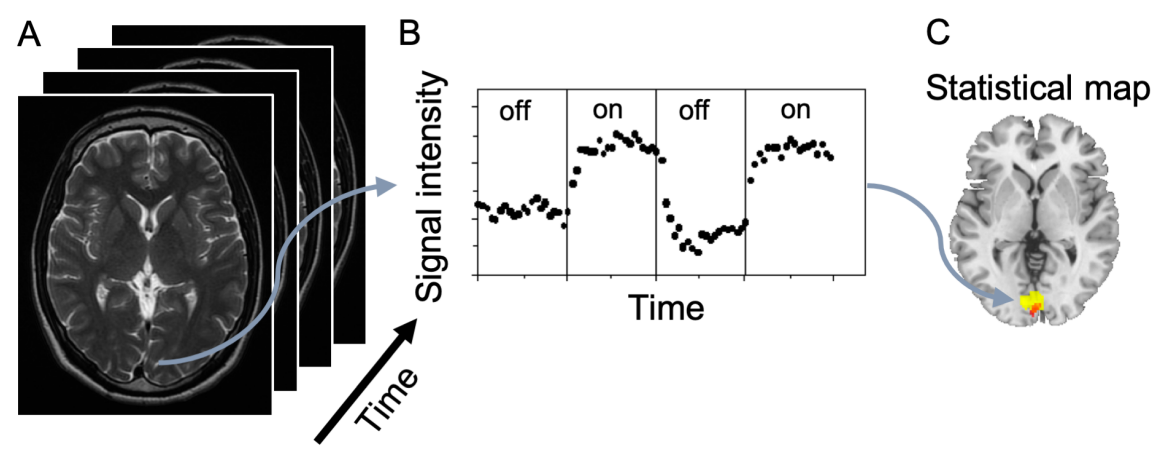

Functional $\left(R_{2}^{*}\right)$ images

Figure 3.3: fMRI: from image to statistical maps. A: $R_{2}^{\star}$ weighted images, which evolves over time. B: Plotting the signal from one cortical region over time yields a time series which might vary with a stimulation ('off' and 'on'). C: Statistically significant differences in the time series between 'off' and 'on' are conventionally shown overlaid on an anatomical image. $R_{2}^{*}$ image adapted from [117], with permission. Middle time course adapted from [118], with permission.

in vivo, with the contrast of the image being dependent on blood oxygenation (Fig. 3.3, A). This study introduced the term BOLD-fMRI imaging, and spawned the research field of fMRI. Shortly thereafter, multiple research groups published findings illustrating the use of BOLD-fMRI imaging to map human brain function $[29,118,121-124]$.

BOLD-fMRI is heavily dependent on the NVC, which enhances the internal contrast (blood oxygenation) upon increases in neuronal activity. Given a stimulus, such as a visual stimulation, spatially localized increases of CBF, $\mathrm{CBV}$, and $\mathrm{CMRO}_{2}$ occurs (with $\triangle \mathrm{CBF}>\Delta \mathrm{CMRO}_{2}$ as discussed in Section 2.2) in some cortical regions. These increases can be seen in the archetypal BOLD response to a brief stimulation (depicted in Fig. 2.6A), and it consists of three distinct characteristics: a debated initial dip (see [125, 126]), an overshoot of increased oxygenation, and finally, a post-peak undershoot. These characteristics constitute the qualitative behavior, which is separate from the quantitative (i.e., measured values) nature of the response. This overshoot of blood oxygenation is reliably captured, increasing the contrast of the captured BOLD image between 'off' and 'on' conditions (Fig. 3.3, B). Correlating the captured experimental signals with the archetypal response patterns renders a statistical map of the cortical regions, which were selectively activated by the stimulus (Fig. 3·3, C).

Since those first reports, BOLD-fMRI has become one of the most widely used non-invasive techniques for investigating brain function in both research and clinical practice. This is due to BOLD-fMRI being non-invasive, its use of an internal contrast agent instead of an injected contrast agent, and 
producing a relatively good spatial and temporal resolution. Despite the wide use, there are a number of important limitations to be considered with BOLDfMRI. For instance, the BOLD signal is a weighted sum given by changes in $\mathrm{CBF}, \mathrm{CBV}$, and $\mathrm{CMRO}_{2}$, which vary simultaneously in the cortical regions. Therefore, it is hard to propagate changes in the BOLD signal to the underlying neural physiology or the neuronal circuitry in the cortical regions. Nevertheless, different methods have been developed to quantify $\mathrm{CMRO}_{2}$ changes from BOLD-fMRI measurements, often denoted 'calibrated fMRI' [61, 127]. However, these methods rely on theoretical frameworks based on a number of assumptions and limitations (see [128] and references therein).

\section{Measuring CBF and CBV using fMRI}

MRI can also be used to non-invasively measure CBF and CBV. For instance, $\mathrm{CBF}$ can be measured using arterial spin labeling techniques, which utilize blood water as a 'tracer'. This is done in a series of steps. First, blood in the neck arteries is magnetically labeled. This labeled portion of blood is allowed to flow into the region the interest, generating a 'tagged' image of the region, as it includes signal from both the tagged blood and the static brain tissue in the region. Subsequently, a control image of the region is acquired without any magnetically labeled blood, so that the image only contains signal originating from the static brain tissue. The contribution from blood can now be calculated by subtracting the control image from the tagged image, producing a perfusion-weighted image. Nevertheless, the specific details of these steps vary with different implementations [129-131]. Data generated using this technique can be found in Paper IV.

Changes in CBV can be assessed using Vascular-Space-Occupancy (VASO) fMRI (see review in [132] and references therein). The VASO sequence builds on the fact that $R_{1}$ relaxation rate differs between tissue and blood. This can be exploited by applying an inversion pulse before the signal acquisition, which essentially nulls the contribution of blood to the acquired signal, but allows for a signal originating from tissue to be detected. The relative changes in the tissue-weighted signal are then associated with changes in CBV. To illustrate this, consider that the fractional composition of the voxel is 0.95 tissue and 0.05 blood. If CBV increases by $20 \%$, the new fractions become 0.94 and 0.06 for tissue and blood, respectively, implying $\mathrm{a} \approx 1 \%$ decrease in the captured tissue signal. Thus, the signal is proportional to 1-CBV [132, 133]. The conventional method is sensitive to the total CBV change, but subsequent improvements to the method can obtain volume changes on the level of arterial and venous compartments (see [134] for derivation). Data generated using this technique can be found in Paper IV. 


\section{Optical imaging-based modalities}

Optical imaging is conventionally employed to observe and measure a broad range of different modalities pertaining to the NVC in rodents in vivo [135]. Two-photon laser scanning microscopy is one prominent technique. This technique can be used to measure the velocity of red blood cells in blood vessels, or to directly measure changes in vessel diameter with excellent spatial, temporal, and depth resolution (see $[88,136,137]$ for examples of application). Nevertheless, only a small number of vessels can be scanned at once. Experimental data consisting of vessel diameter changes are used in paper III \& IV.

As with MRI, hemoglobin exhibits different properties with and without bound oxygen which can be detected using optical imaging, often denoted optical imaging of 'intrinsic signals' [135, 138]. More specifically, the absorption of light changes depending on if hemoglobin is bound to oxygen or not. This technique allows one to observe changes in oxy- and deoxygenated hemoglobin concentration. Furthermore, the sum of these changes reflects the change in total hemoglobin present in the measurement site, which can act as a proxy for changes in blood volume in the measured area. Experimental data capturing hemoglobin changes is used in paper IV.

One limitation with both of these optical techniques is that these techniques are affected by wavelength scattering in the tissue, limiting the experimental resolution (see reviews in $[135,139,140]$ ). Light in the visible spectrum is often used for data acquisition in both of techniques, which is strongly scattered by the bone of the skull. Therefore, these experiments are only performed in animals which have had a cranial window inserted into the skull, or with a genetically manipulated thin skull bone [135].

Non-invasive measurements of hemoglobin concentrations in humans can be performed using NIRS [141, 142]. This is due to weak absorption of near-infrared light by hemoglobin [135] and a small scattering in tissue and skull [143]. Nevertheless, NIRS suffers from low spatial resolution and low depth penetration, only capturing hemoglobin changes at the surface of the cortex. While fMRI has a higher spatial resolution and captures the whole brain at once, NIRS is a portable instrument with low noise during data acquisition. For all these reasons, NIRS is commonly used in a more clinical setting, for instance, in studies on infants or conversation [144-146].

\section{Optogenetic stimulation}

Optogenetics (OG) is an experimental technique that allows for a 'switchlike' control of neurons through the insertion of light-sensitive ion channels into the plasma membrane $[147,148]$. The ion channel, Channelrhodopsin- 
2 (ChR2) [149], opens when blue light illuminates the channel but does not respond to any other stimulation (e.g., different wavelength, electrical stimulation). Thus, the neuron can be excited through the use of light pulses [150]. Typically, the channel is selectively expressed in a targeted subset of neurons, through the use of viral transduction methods (such as in $[88,92,96])$. This enables the selective activation of specific neurons, allowing for investigations of neuron-specific contributions to the evoked vascular response [112]. Data generated using OG perturbations are used in Paper III \& IV.
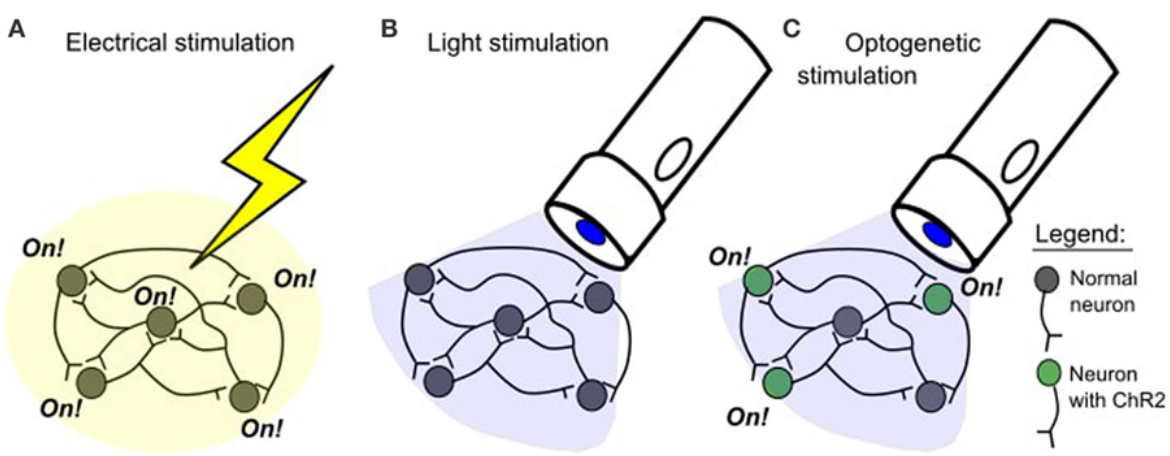

Figure 3.4: Simple introduction to optogenetics. A: A conventional electrical stimulation will evoke a response from all neuronal cells within the cortical area. B: Illuminating a cortical area which lacks ChR2 expression with blue light will not evoke a response. C: Blue light opens the ChR2 channel, evoking a neuronal response in those neurons that express ChR2. Image taken from [150], with permission.

\subsection{Pharmacological modulations of the NVC}

To study the NVC and its pertaining aspects in vivo, scientists often employ the use of animal studies, and most of our current understanding regarding the inner workings of the brain stem from this practice. Nevertheless, one major issue (besides from translation from animal to human) is the nonvoluntary task conundrum (see [151] and references therein). In contrast to voluntary humans, animals lack interest in participating in experimental studies. This issue can be circumvented through the use of head fixation, body restraint, habituation, behavioral training (especially to the noise of the MR gradients), and lastly, the use of anesthetics. The commonly used anesthetics within NVC research exert a broad range of effects on neurons and other neural cells, but can alleviate potential suffering due to the experimental setup. Nevertheless, these effects include: changes in neuronal excitability, vascular reactivity, cerebral metabolism, and other physiological processes (see reviews in $[152,153]$ and references therein). Anesthetics typically exert their mechanism of action directly on ion channels, for instance on the $\mathrm{GABA}_{\alpha}$ receptor, the NMDA receptor, or on different types of $\mathrm{K}^{+}$channels (see review 
in $[154,155])$. However, how the action of different anesthetics propagate from altered ion-channel function to the conventionally measured NVC observables such as electrode measurements, $\mathrm{CBF}, \mathrm{CMRO}_{2}$, and blood oxygenation remain to a large extent unknown, due to the fact that few studies include both an awake and an anesthesia cohort.
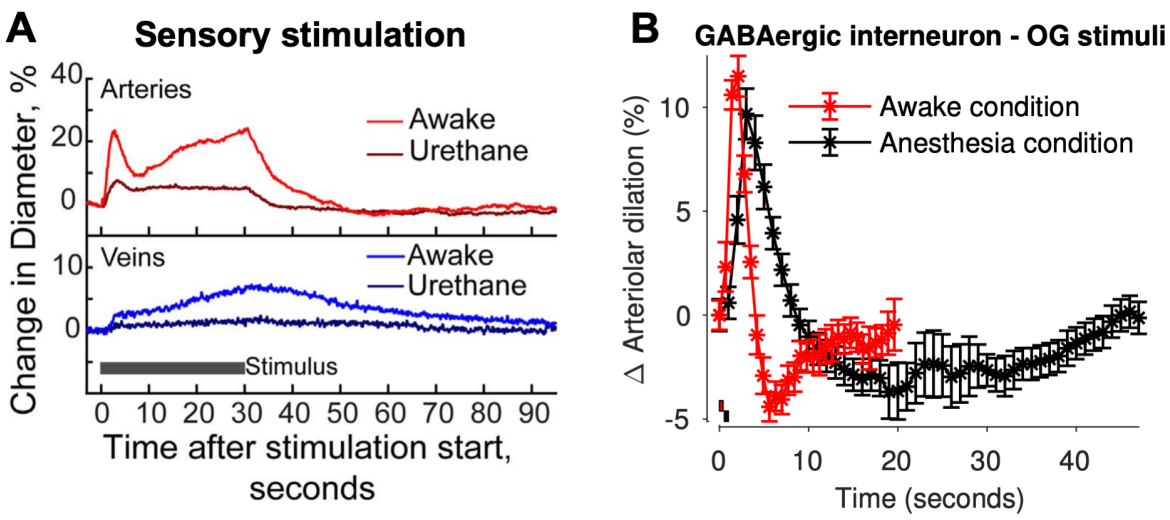

Figure 3.5: Two examples illustrating the effects of anesthetics on observed hemodynamic responses. Comparison of dilation of arterioles/arteries (A, B) and veins (A) captured in the somatosensory cortex for awake and anesthetized mice from two studies: Drew et al., (A) [137] and Uhlirova et al., (B) [88]. A: Sensory stimulation consisting of air puffs aimed at the vibrissae with and without the administration of urethane. The administration of urethane decreases arterial dilation and removes the bi-modal response. The venous dilation is completely abolished. Image taken from [153], with permission. B: Brief optogenetic stimulation of GABAergic interneurons with and without administration of the anesthetic $\alpha$-chloralose. A prolonged post-peak undershoot in the arteriolar diameter is observed during anesthesia. Image adapted from Paper III, with permission. Experimental data originally published in [88].

Nevertheless, a few studies exist [88, 137, 156-158]. The overall picture from these studies suggests that while anesthesia reduces neural activity, the hemodynamic measures (CBF, CBV, blood oxygenation) are suppressed to a greater extent relative to neural activity [153]. Furthermore, the quantitative relationship between neural response and hemodynamic response is highly dependent on the type and dosage of the anesthetic used [159-162]. For instance, Pisauro et al., measured the spiking of neurons using multiunit activity and CBV responses in awake and anesthetized mice [158]. For the awake condition, the spiking rates were $(\approx 20 \%)$ higher relative to anesthesia, but the $\mathrm{CBV}$ response was increased by $\approx 110 \%$, relative to anesthesia. A similar disproportionate change in hemodynamic contra neural response has been observed when instead capturing local field potentials (LFP) in mice $[156,157]$. Another study, Drew et al., reports arteriolar and venular diameter responses during both awake and urethane conditions [137]. The use of urethane significantly reduces the arterial dilation, removing a bi-modal peak 
response observed during awake condition and abolishes the venous dilation (Fig. 3.5A). In addition, Uhlirova et al., reports a prolonged post-peak undershoot in arteriolar diameter during anesthesia, using an optogenetic stimulation of GABAergic interneurons [88] (Fig. 3.5B). Finally, anesthesia affects the timing of the hemodynamic responses across all species, causing a delay in the hemodynamic response as compared to awake $[156,158,163]$.

The anesthetic used to capture the experimental data used in paper III (see [88] for original data publication), $\alpha$-chloralose, is commonly used in rodent based research on the NVC as it preserves a robust hemodynamic response to sensory stimulation $[164,165]$. It is known that $\alpha$-chloralose potentiates the $\mathrm{Cl}^{-}$ion conductance through the $\mathrm{GABA}_{\alpha}$ channel by increasing the binding affinity of GABA [166]. Other studies on the physiological effects of $\alpha$ chloralose suggests reduced baseline $\mathrm{CBF}$ values [152], increased blood $\mathrm{pH}$, and decreased heart rate [167]. A recent study showed that VSM lacks the expression of the $\mathrm{GABA}_{\alpha}$ receptor-channel complex [168], which suggests that $\alpha$-chloralose does not directly act on the vasculature.

Together, these studies show that anesthetics significantly alter the hemodynamic responses commonly observed in the NVC, with the alterations being both dependent on the anesthetic used, the type of stimulation used, as well as the dosage of the anesthetic. For all these reasons, the use of anesthetics within the NVC research is an ongoing debate, and more studies of NVC related observables in rodents that are performed without anesthesia are emerging. 


\section{4}

\section{Mathematical Modeling in Systems Biology}

Mathematical modeling is at the core of the research field Systems Biology. In Systems Biology, mathematical models are used to provide a quantitative understanding of complicated biological processes [36], and to unravel or provide insights of properties that cannot be assessed or measured directly using experimental techniques [169]. The use of mathematical models is widespread across many different organs and diseases. The size and scope of the models range from mechanistic models, describing the dynamics of one or two proteins, to genome-wide models within bioinformatics. A common application within mechanistic modeling is hypothesis-based testing, where hypotheses describing the mechanisms which govern the biological system are evaluated and compared [170, 171], which in some cases leads to the rejection of a hypothesis [171]. Models within bioinformatics are typically based on omics data, where the structural understanding is often fuzzier, making the approach and conclusions more data-driven. Through this broad spectrum of use, mathematical models have emerged as powerful tools for developing model-based treatment strategies for various diseases or as a platform for model-based decision making [172].

Different types of mathematical models in Systems Biology exist, such as deterministic models which feature no randomness [173], or stochastic models which are affected by random processes [174]. The choice of model type depends on the question at hand, as well on the biological system with its pertaining measurement observables under consideration. For studies that analyze the average behavior of a population over time, such as in this thesis, models based on deterministic differential-algebraic equations (DAEs) and ordinary differential equations (ODEs) are often employed. The main focus 
of this chapter is to describe mathematical modeling in the context of differential equations. Nevertheless, some of the methods are relevant for other types of modeling frameworks, such as the iterative cycle of mathematical modeling.

\subsection{Iterative cycle of mathematical modeling}

As touched upon in Section 2.3, mathematical modeling of mechanisms rests on two core pillars: 1) model-based analysis of experimental data and 2) the use model-derived insights or predictions to validate the model by new experimental designs. These pillars form the basis of the iterative cycle of mathematical modeling, illustrated in Figure 4.1. This modeling cycle constitutes the practical framework for model construction, model-based hypothesis testing and data analysis, and finally, model predictions and modelderived insights or decisions. This framework is valid on two scales: on a small scale, concerning the individual modeler who performs the analyses, and on a larger group scale, suggesting how to organize an iterative collaboration between experimentalists who gather data and modelers who make the models [39].

Throughout this chapter, we will carefully examine some important aspects of the individual key steps in the modeling cycle (Fig. 4.1). I have chosen to focus on seven topics: model simulation, likelihood functions, parameter estimation, assessing goodness of fit, identifiability of parameters, and finally, the use of validation data for model selection.

\subsection{Ordinary and algebraic differential equations}

Throughout this thesis, all models are formulated using ODEs (Paper I-III) or DAEs (Paper IV). The general notation of an ODE model is as follows:

$$
\begin{aligned}
\dot{x} & =f(x, p, u, t) \\
x\left(t_{0}\right) & =x_{0}(p) \\
\widehat{y}(t, p) & =g(x(t, p, u), p)
\end{aligned}
$$

in which $x$ are the state variables, describing the concentration or amount of an entity with respect to time; where $\dot{x}$ represent the derivative of the states with respect to time; where $f$ and $g$ are non-linear smooth functions; where $p$ is the vector of unknown time-independent parameters; where $u$ is the input signal associated with the experimental data, if applicable; where $x\left(t_{0}\right)$ are the initial values of the states at $t=t_{0}$, which are dependent on the parameters $x_{0}(p)$; and finally, where $\hat{y}$ are the model outputs corresponding to the measurement observables. Note that $x, u$, and $y$ depend on time $(t)$. 


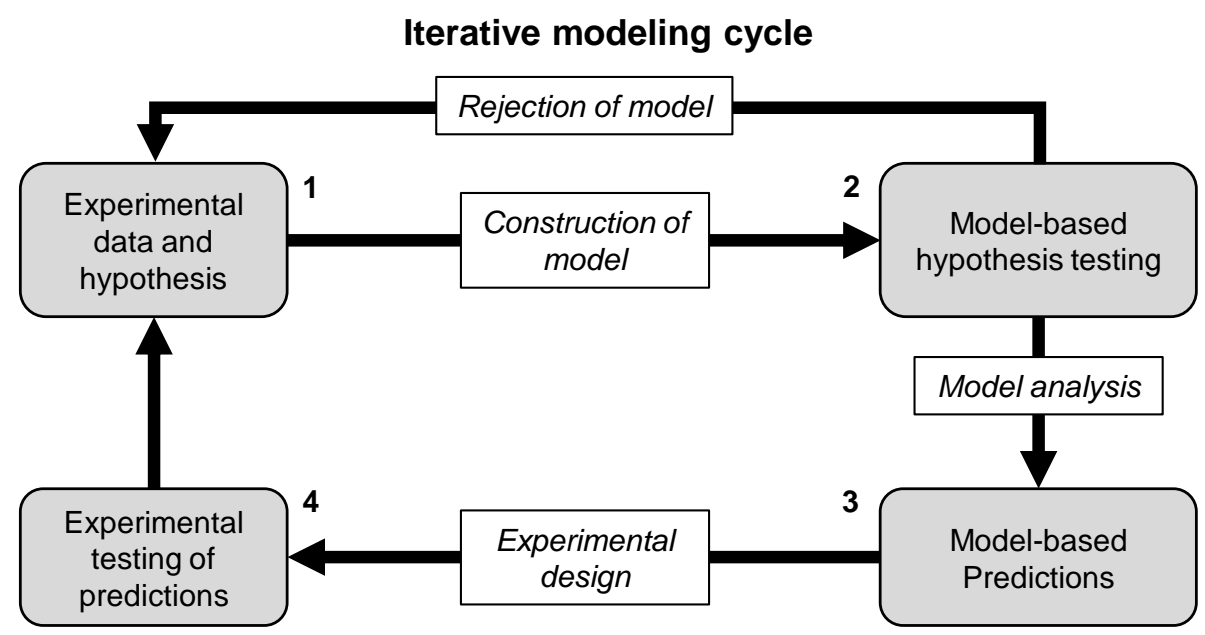

Figure 4.1: The iterative modeling cycle used in this thesis. (1) Combine experimental data captured from the biological system at hand with knowledge and hypotheses regarding the system, and construct a mathematical model. (2) Perform model-based hypothesis testing by fitting the model to the experimental data. This has two outcomes: a) if the model cannot describe the data, then reject the model; or b) if the model can describe data, it is passed on to the next step. If a), go back to the model construction and alter the model or hypothesis. If b), proceed to (3), where identifiable model predictions with low uncertainty are generated through model analysis. These predictions are favorably ones that can be experimentally validated or be used to design new experimental studies. (4) Validate the model against data of the model predictions or any other independent data that can be used for validation. If the model predictions do not overlap with validation data, start over at (1). Conversely, if the predictions match the validation data, the hypothesis is tentatively accepted. This loop can be iterated many times, each time refining the model further and expanding the mechanistic understanding of the system.

A DAE is the generalized case of an ODE, where the derivatives of some states $x$ are unspecified, denoted $z . z$ is instead solved for every time point through the use of algebraic relationships with $x$ and $t$. These systems usually arise in e.g. electrical circuits. For further details of DAEs, I refer the reader to [175]. The general notation of a DAE is given by:

$$
\begin{aligned}
F(\dot{x}, x, z, p, u, t) & =0 \\
F\left(\dot{x}\left(t_{0}, p\right), x\left(t_{0}, p\right), z\left(t_{0}, p\right), t_{0}\right) & =0 \\
\hat{y}=g(x, z, p, u, t) &
\end{aligned}
$$

where $z$ represents the states for which the derivative function is unknown and $F$ corresponds to the non-linear function of the system.

\subsection{Model simulation}

The lion's share of ODE models is solved ('simulated') numerically by the use of different step-based methods. These numerical methods are often divided 
into two categories: one-step and multi-step solvers. One-step solvers, such as the well-known Euler step [176] (developed by the mathematician Leonhard Euler in the late 1760s), only require information about the system from the preceding step to calculate the next step. In contrast, multi-step solvers make use of multiple sequential points, which are weighted together to calculate the next step. Some methods are explicit, meaning that the value of the system at the next step is a function of the value at the present or previous steps. Other methods are implicit, such as backward differentiation formulas. An implicit solver calculates the solution for the next step by solving equations containing both the current and future steps of the system. These implicit methods are typically used if the ODE system is stiff and most conventional ODE solver packages, such as the SUNDIALS package [177], employ these implicit solvers. To practically illustrate a simulation, I will go through the classical explicit, multi-step method for ODE solving: the 4th order RungeKutta (RK4) approximation. RK4 was developed by the mathematician's Carl Runge and Martin Willhelm Kutta during the late 180os [178, 179]. First, consider the following initial value problem:

$$
\dot{x}=f(t, x), \quad x\left(t_{0}\right)=x_{0} .
$$

where $\dot{x}$ is the derivative of the variable $x$ as given by the function $f$ (which is dependent on time $t$, and $x$ ) and the initial value of $x, x\left(t_{0}\right)$, is given by $x_{0}$. The variable $x$ can be calculated using $\mathrm{RK}_{4}$ by:

$$
\begin{array}{cc}
x_{n+1}=x_{n}+\frac{h}{6} & \left(k_{1}+2 k_{2}+2 k_{3}+k_{4}\right) \\
t_{n+1} & =t_{n}+h
\end{array}
$$

where $x_{n+1}$ is the RK4 approximation of $x\left(t_{n+1}\right)$, which is determined by the present value $\left(x_{n}\right)$ and an estimated slope, given by the weighted combined sum of $k_{i}, i \in 1, . ., 4$, multiplied by the size of the temporal step $(h)$. Explicitly, the slope $k_{i}$ is given by:

$$
\begin{array}{cc}
k_{1} & =f\left(t_{n}, x_{n}\right) \\
k_{2}=f\left(t_{n}+\frac{h}{2}, x_{n}+h \frac{k_{1}}{2}\right) \\
k_{3}=f\left(t_{n}+\frac{h}{2}, x_{n}+h \frac{k_{2}}{2}\right) \\
k_{4}=f\left(t_{n}+h, x_{n}+h k_{3}\right)
\end{array}
$$

To simplify the meaning of $k_{i}$, a geometric representation is presented in Figure 4.2. Using this figure and Equation 4.5, one can observe that $k_{1}$ is based on the derivative of the starting point $t_{n}$, which is simply an Euler's step. $k_{2}, k_{3}$ are the derivatives at the mid-point of the step interval $\left(t_{n}+\frac{h}{2}\right)$. Finally, $k_{4}$ is the derivative at the end of the step interval $\left(t_{n}+h\right)$.

One important consideration with a fixed step method is that the choice of $h$ is non-trivial: 1) selecting a too large $h$, the approximated derivatives 


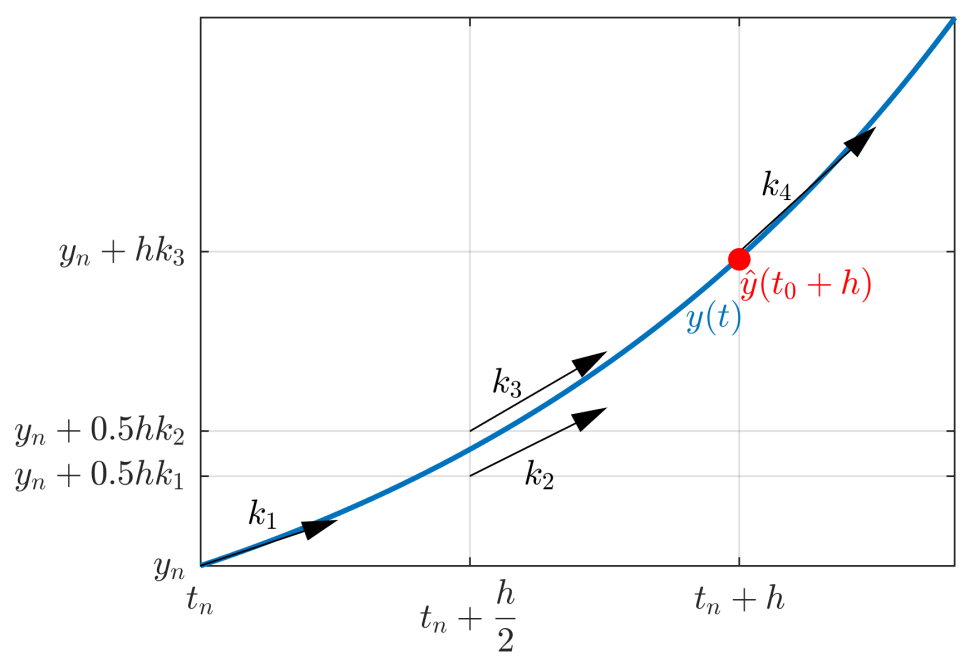

Figure 4.2: Geometric representation of the 4th order Runge-Kutta formula. $k_{1}$ represents the slope at $\left(t_{n}, y_{n}\right)$, calculated using Euler's step. $k_{2}, k_{3}$ are the derivatives at the midpoint of the step interval $\left(t_{n}+\frac{h}{2}\right)$. Finally, $k_{4}$ is the derivative at the end of the step interval $\left(t_{n}+h\right)$.

are no longer a sufficient approximation of the true system; 2) if one instead selects a too small step size, numerical errors will accumulate, and as with 1), will result in an erroneous approximation of the true system. Modern ODE solvers negate this sensitivity to the choice of step size $h$ by using an adaptive step size (see Fig. 4.3).

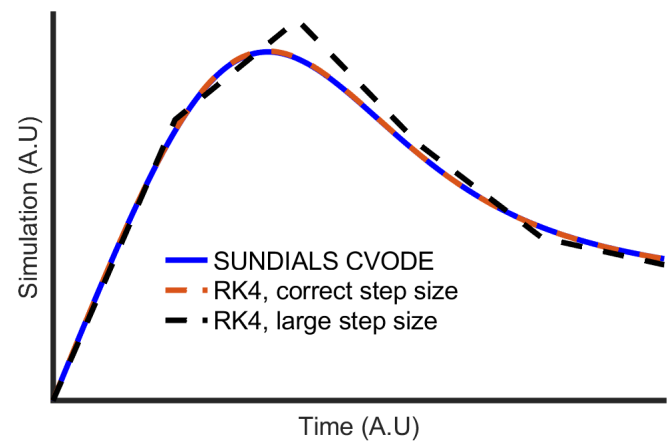

Figure 4.3: Comparison between RK4 and a modern ODE solver within the SUNDIALS package. Too large step size results in an erroneous approximation of the true system. 
Following the typical modeling cycle outlined in Figure 4.1: once a model structure has been formulated, the parameters $p$ which produce a model simulation that as closely as possible replicates observed experimental data, need to be determined. This is done by minimizing the disagreement between experimental data and model simulations. This disagreement is typically evaluated using the likelihood function.

\subsection{Likelihood functions}

Assuming independent, normally distributed additive measurement noise, the likelihood of observing data $y$ given $p$ is:

$$
L(y \mid p)=\prod_{e=1}^{n_{e}} \prod_{o=1}^{n_{o}^{e}} \prod_{d=1}^{n_{d}^{e, o}} \frac{1}{\sqrt{2 \pi} \sigma_{d}^{e, o}} \exp \left(-\frac{1}{2}\left(\frac{y_{d}^{e, o}-\widehat{y}_{d}^{e, o}(p)}{\sigma_{d}^{e, o}}\right)^{2}\right)
$$

where $n_{e}$ is the number of experiments $e$; where $n_{o}^{e}$ is the number of observables $o$ per $e$; where $n_{d}^{e, o}$ is the number of data points $d$ per $o$ and $e$; where $\sigma_{d}^{e, o}$ is the uncertainty of the data point, typically standard error of the mean (SEM); where $y_{d}^{e, o}$ is the measured data point; where $\widehat{y}(p)$ is the corresponding simulated data point. By maximizing $L$, the maximum likelihood estimate of the unknown parameters $p$ is found. In practice, it is more numerically efficient to minimize the equivalent negative log-likelihood function:

$$
J(p)=-\log (L(y \mid p))=\frac{1}{2} \sum_{e=1}^{n_{e}} \sum_{o=1}^{n_{o}^{e}} \sum_{d=1}^{n_{d}^{e, o}}\left[\log \left(2 \pi\left(\sigma_{d}^{e, o}\right)^{2}\right)+\left(\frac{y_{d}^{e, o}-\widehat{y}_{d}^{e, o}(p)}{\sigma_{d}^{e, o}}\right)^{2}\right]
$$

If the measurement uncertainty $\sigma_{d}^{e, o}$ is known, $J(p)$ share the same optimal parameters with the measurement uncertainty weighted sum of squares residual (SSR) $J_{s s r}$ :

$$
J_{s s r}(p)=\sum_{e=1}^{n_{e}} \sum_{o=1}^{n_{o}^{e}} \sum_{d=1}^{n_{d}^{e, o}}\left(\frac{y_{d}^{e, o}-\widehat{y}_{s}^{e, o}(p)}{\sigma_{d}^{e, o}}\right)^{2}
$$

The summation in Eq. 4.7 and Eq. 4.8 of the squared and normalized residuals corresponds to calculating the vertical distance between the simulated values $\widehat{y}_{d}^{e, o}(p)$ and experimentally measured data $y_{d}^{e, o}$.

As a side note, one can easily re-arrange Eq. 4.7 to give the relationship between $J(p)$ and $J_{s s r}$ as:

$$
J_{s s r}(p)=2 J(p)-\sum_{e=1}^{n_{e}} \sum_{o=1}^{n_{o}^{e}} \sum_{d=1}^{n_{d}^{e, o}} \log \left(2 \pi\left(\sigma_{d}^{e, o}\right)^{2}\right)
$$

Local optimization algorithms (see Section 4.5) utilize the gradient of the chosen likelihood function, with respect to the parameters $p_{k}, k=1, \ldots, n_{p}$ 
( $n_{p}$ equals the number of parameters), in order to improve convergence to a local/global minima. Differentiation of $J(p)$ with respect to the parameters $p$ gives:

$$
\frac{d J}{d p_{k}}=\left.\sum_{e=1}^{n_{e}} \sum_{o=1}^{n_{o}^{e}} \sum_{s=1}^{n_{d}^{e, o}} \frac{1}{\sigma_{d}^{e, o}}\left(\frac{1}{2}-\left(\frac{y_{d}^{e, o}-\widehat{y}_{d}^{e, o}(p)}{\sigma_{d}^{e, o}}\right)^{2}\right) \frac{d \sigma_{d}^{e, o}}{d p_{k}}\right|_{(p)}-\frac{y_{d}^{e, o}-\widehat{y}_{d}^{e, o}(p)}{\left(\sigma_{d}^{e, o}\right)^{2}} s_{o, k}^{y, e}(d)
$$

Where $s_{o, k}^{y, e}(d)$ denotes the sensitivity of the output $\widehat{y}_{d}^{e, o}(p)$ at time point $d$ with respect to parameter $p_{k}$. To note: if the experimental data uncertainty is parameter independent, i.e., $\frac{d \sigma_{d}^{e, o}}{d p_{k}}=0$, the first term of the expression is eliminated. To calculate the likelihood gradient, several method exists, such as finite differences and forward or adjoint sensitivity analysis.

\section{Finite differences}

Finite differences gives an approximation of the objective function gradient with respect to $p_{k}$ and is given by:

$$
\frac{d J}{d p_{k}} \approx \frac{J\left(p+a e_{k}\right)-J\left(p-b e_{k}\right)}{a+b}
$$

where $a, b \geq 0$ and $e_{k}$ is the $k$ th element in an unit vector of the parameters. In practice, three approaches to calculating this approximation are used: forward ( $a=\epsilon, b=0)$, backward $(a=0, b=\epsilon)$ or central $(a=\epsilon, b=\epsilon)$ differences, where $\epsilon$ is the parameter perturbation factor. Such a calculation is performed in three steps (see [180]):

1: The model is numerically simulated, giving the state $x(t, p)$ and observable output $\widehat{y}(t, p)$ trajectories.

2: The model is repeatedly re-simulated, each time perturbing one element of the parameter vector $p^{(k)}=p+\epsilon e_{k}$ for $k=1, \ldots, n_{k}$, resulting in new state and output trajectories for each perturbation $\left(x\left(t, p^{(k)}\right)\right.$ and $\left.\widehat{y}\left(t, p^{(k)}\right)\right)$.

3: The gradient $\frac{d J}{d p_{k}}$ is calculated from the output $\widehat{y}(t, p)$ and the perturbed output $y\left(t, p^{(k)}\right)$ using Eq. 4.11.

One can note that the method of forward and backward differences requires $n_{k}+1$ function evaluations (simulations), while central differences requires $2 n_{k}$ function evaluations. This makes the calculation of the gradient time consuming for large models.

\section{Forward and adjoint sensitivity analysis}

A method common within physics and classical engineering is to numerically solve for the sensitivity of the observables $s_{o, k}^{\widehat{y}}(d)$ during the simulation. This 
can be done by differentiating Equation 4.1, giving the following expressions:

$$
\begin{aligned}
\dot{s}_{k}^{x} & =\frac{d f}{d x} s_{k}^{x}+\frac{d f}{d p_{k}}, \quad s_{k}^{x}\left(t_{0}\right)=\frac{d x_{0}}{d p_{k}} \\
s_{k}^{\widehat{y}} & =\frac{d g}{d x} s_{k}^{x}+\frac{d g}{d p_{k}}
\end{aligned}
$$

where $s_{k}^{x}$ is the sensitivity of state $x$ with respect to $p_{k}$. This analysis is also performed in three steps (see [180]):

1: As previously, trajectories of the states $x(t, p)$ and observables $\widehat{y}(t, p)$ are calculated through simulation.

2: Using the state trajectory $x(t, p)$, the state sensitivities $s_{k}^{x}$ and output sensitivities $s_{k}^{\widehat{y}}$ can be calculated for all parameters $k=1, \ldots, n_{p}$.

3: Finally, the objective function gradient $\frac{d J}{d p_{k}}$ can then be calculated from $\widehat{y}(t, p)$ and $s_{k}^{\widehat{y}}$ using Equation 4.10.

One limitation with using forward sensitivity analysis is that calculating $\frac{d J}{d p_{k}}$ using Eq. 4.10 requires the solution of Eq. 4.12. This is not an issue for small models with a few parameters or states, but it becomes computationally cumbersome for large models with thousands of parameters or states. However, it is possible to derive an expression of the gradient of the likelihood function that does not depend on the state sensitivities $s_{k}^{x}$, circumventing this issue. In short, one can introduce an adjoint state which is solved via a backward (meaning the state evolves backwards in time) differential equation (see [180] for detailed derivation). The key difference between adjoint and forward sensitivity analysis is that the state and output sensitivities $\left(s_{k}^{x}\right.$, $s_{k}^{\widehat{y}}$ ) are not explicitly calculated. Instead, $\frac{d J}{d p_{k}}$ is directly calculated by a solving $n_{p}$ one-dimensional integration problems over short time intervals [180]. In practice, this means that computational time required to calculate the likelihood function gradient using adjoint sensitivity analysis is only weakly dependent on the number of parameters in the model [180].

Figure 4.4 visualizes the comparison between these three methods: finite differences (blue line), forward sensitivity (orange line), and adjoint sensitivity (yellow line). As seen, for a small number of parameters (x-axis), the three different methods share a similar computational time (y-axis). However, for models with increasingly more parameters, forward and most prominently adjoint sensitivity analysis has a much shorter computational time, as compared to finite differences.

Forward and adjoint sensitivity analysis has been implemented in the 'Advanced Matlab Interface for CVODES and IDAS' toolbox [180, 181], which is 
extensively employed throughout Paper III and IV for model simulations and gradient calculations.

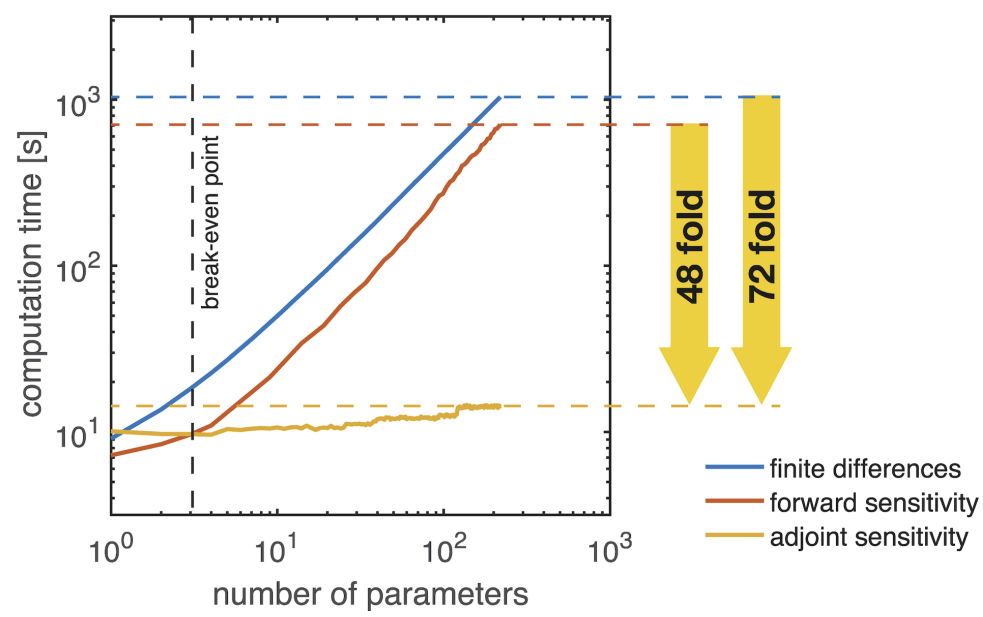

Figure 4.4: The computational time of likelihood gradient calculation with respect to the number of parameters using three different methods. The computational time for finite differences and forward sensitivity analysis increases linearly with respect to number of parameters, while adjoint sensitivity analysis is only weakly linearly dependent on the number of parameters. Picture taken from [180], with permission.

\subsection{Optimization - minimization of the likelihood function}

With a suitable likelihood function chosen in the previous section, the next logical step is to locate the global minima in the typically multi-dimensional likelihood landscape. In other words, finding the parameters $\hat{p}$ which minimizes the negative log-likelihood function $J(p)$ (Eq. 4.7) or the SSR function $J_{s s r}(p)$ (Eq. 4.8). This is commonly denoted as:

$$
\hat{p}=\arg \min J(p)
$$

To search for the global minimum in the likelihood landscape (see Fig. 4.5 for a simple one parameter case), you typically employ one or several different available optimization algorithms or techniques. Generally, optimization techniques can be divided into two categories: local optimization and global optimization.

\section{Local optimization}

Multiple local optimization algorithms exist. A typical hallmark feature of local optimizers is that these typically are deterministic, i.e., given a specific 


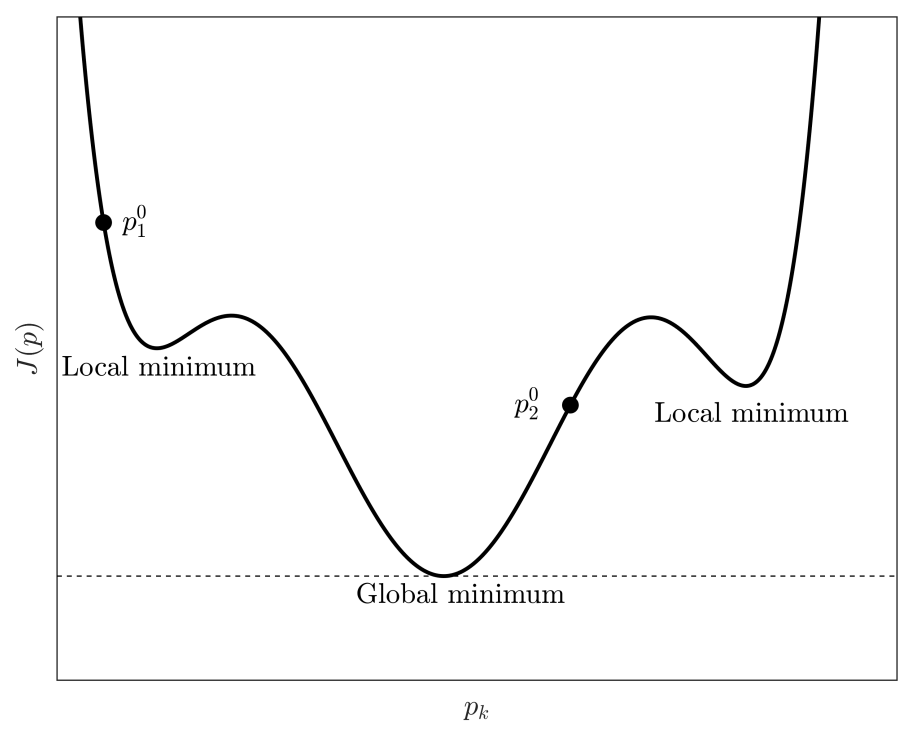

Figure 4.5: The negative log-likelihood function landscape for a simple one parameter system. In this case, a global minimum exists, as well as two local minima. Two starting points, $p_{1}^{0}$ and $p_{2}^{0}$, are initiated in the landscape. Generally, local optimization strategies will not search in an upwards direction, missing the global minimum if starting from $p_{1}^{0}$. In contrast, using $p_{2}^{0}$ as a starting point, the local optimization will converge to the true (global) optima. For a global optimization strategy, the algorithm can travel in an upwards direction, and will eventually converge to the global optima. Nevertheless, choosing a global strategy comes with the trade-off of higher computational costs and varying performance, as the global techniques typically include stochastic elements.

starting point $p^{0}$, the individual optimizer will reach the same solution every time. Generally, a local optimization strategy locates the local minima in the neighborhood of the starting point $p^{0}$, typically guided by the derivative of the negative log-likelihood function (see Section 4.4). As such, if we look at Figure 4.5 again, we see that given the starting point $p_{1}^{0}$, a local optimization strategy will only find the left local minima, not the global minima. In contrast, initiating a local search at $p_{2}^{0}$ will result in locating the global minima. Therefore, local optimization techniques are heavily influenced by the start guess $p^{0}$.

Below, I will go through two common local optimization methods: the simple gradient descent algorithm, and the more elaborate Newton method. In gradient descent, the algorithm iteratively steps in the negative direction 
of the chosen likelihood function gradient until it fulfills the termination criteria, such as successful convergence or number of max iterations reached.

$$
p^{n+1}=p^{n}-h \frac{d J\left(p^{n}\right)}{d p}
$$

where $h$ is the step size and $\frac{d J}{d p}$ is the gradient of the chosen likelihood function (Eq. 4.10). Note that this $h$ is not the same as in Eq. 4.4, as there we step in the state space of $x$, while here we step in the parameter space of $p$. Gradient descent converges linearly, which is slower than other methods such as Newton or quasi-Newton methods. These methods incorporate the curvature of the likelihood function to quadratically converge to extreme points. Nevertheless, these methods require the calculation of both the gradient and the Hessian $H$, which are the second-order derivatives of the chosen likelihood function:

$$
\begin{aligned}
p^{n+1}= & p^{n}-h \frac{d J\left(p^{n}\right)}{d p} \frac{1}{H\left(p^{n}\right)} \\
\text { where: } & H\left(p_{i, j}^{n}\right)=\frac{d^{2} J\left(p^{n}\right)}{d p_{i} d p_{j}}
\end{aligned}
$$

In Figure 4.6, a graphical comparison of the two local optimizers discussed above are shown (gradient descent as the purple line, and the Newton method as the gray line). Newton method takes a more direct path to the solution point $\hat{p}$ by utilizing the gradient and Hessian information. Nevertheless, the computation of the Hessian is cumbersome and non-trivial depending on the model complexity. Therefore, quasi-Newton methods utilize approximations of the Hessian, which are less precise but has a reduced computational cost. For more detail on approximating the Hessian, I refer the reader to Section 4.4 for a summarized view on available methods for calculating these gradients and to [182] for an in-depth view.

The main drawback of local optimization strategies is that the probability of getting stuck in local minima increases for highly complex or parameterized models. Nevertheless, multi-start optimization has been suggested to at least partly remedy this drawback (see [184] and references therein). Multistart optimization works by dividing the parameter space into even grids, placing starting points in these grids (for instance using Latin Hypercube Sampling [185]). Next, a local optimization is launched for each point. This however becomes infeasible for highly parameterized systems, as you conventionally use $m^{n}$ starting points, where $m$ is the the number of grids, and $n$ is the number of parameters. Nevertheless, this approach has been shown to perform well even for large models when coupled with precise calculations of the likelihood gradients [186]. 


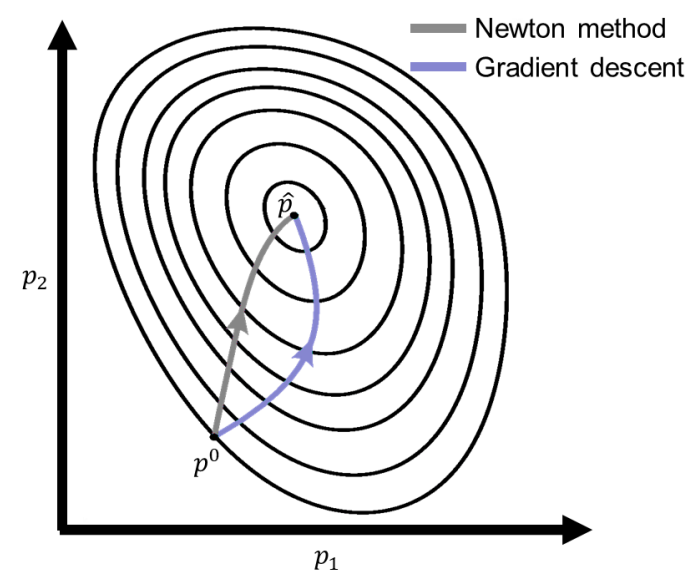

Figure 4.6: The iterative solution path taken for the two compared local optimizers: gradient descent (purple line) and Newton method (gray line). As seen, the Newton method converges through a shorter path to the minima point $(\hat{p})$ from the starting point $p^{0}$ as it exploits the curvature of the likelihood (black contour lines) for a more direct path. In contrast, gradient descent converges by iteratively stepping orthogonally to the likelihood contours. Picture modified with permission from [183].

\section{Global optimization}

Global optimization methods are designed to circumvent the major drawback with local optimization: local methods can only search downhill in the likelihood landscape. This means that these methods are unable to continue searching if stuck in local minima (see Fig. 4.5). Therefore, global optimization algorithms include a way of escaping local minima, thus being able to climb uphill in the likelihood landscape and continue to the search for favorable parameter points. This is often achieved by including a stochastic process (randomness) in the optimization algorithm, meaning a different solution can be found every time the algorithm is executed, despite using identical starting points (assuming unsuccessful global convergence). These methods are typically referred to as metaheuristic in the numerical optimization field [187]. In the work presented in this thesis, two global optimization methods have been extensively used: Simulated Annealing $[188,189]$ in Paper I \& II, and a Scatter Search $[190,191]$ based method (see $[192,193]$ ) in Paper III \& IV. Below, I will briefly go through the core aspects of these two methods.

\section{Simulated annealing}

Simulated Annealing (SA) is a global metaheuristic algorithm introduced by Kirkpatrick et al. in 1983 [189]. SA mimics the basic concept of the metallurgic process of annealing, where metal is repeatedly heated and cooled, which removes imperfections in the crystalline structure of the metal, and al- 
lows the atoms to adopt their lowest energy conformations $[173,189]$. In SA, this process is translated to the key variable temperature. The temperature $T$ controls the probability $P$ of the algorithm to a new candidate solution of the parameters $p^{\prime}$, as compared to the current or optimal solution $p$, and this probability is in [189] expressed as:

$$
P\left(p^{\prime}, p, T\right) \begin{cases}1 & \text { if } J\left(p^{\prime}\right)-J(p)<0 \\ e^{\frac{-\left(J\left(p^{\prime}\right)-J(p)\right)}{T}} & \text { otherwise }\end{cases}
$$

where $P\left(p^{\prime}, p, T\right)$ is the probability of accepting the move with respect to $p^{\prime}, p$, and $T$. As seen, the move is always accepted if $J\left(p^{\prime}\right)-J(p)<0$. Typically, SA starts with a high $T$, meaning that the algorithm will accept large moves (uphill) in the likelihood landscape, allowing for a global search. The temperature is decreased iteratively, refining the found candidate solutions, with a decreased probability of accepting large uphill moves. This mechanism of accepting worse solutions is paired generation of a new candidate solution (based on the current solution), which essentially choose a neighboring point. This loop is completed when the temperature reaches zero, allowing no uphill moves to be made and thus acts as a local optimizer.

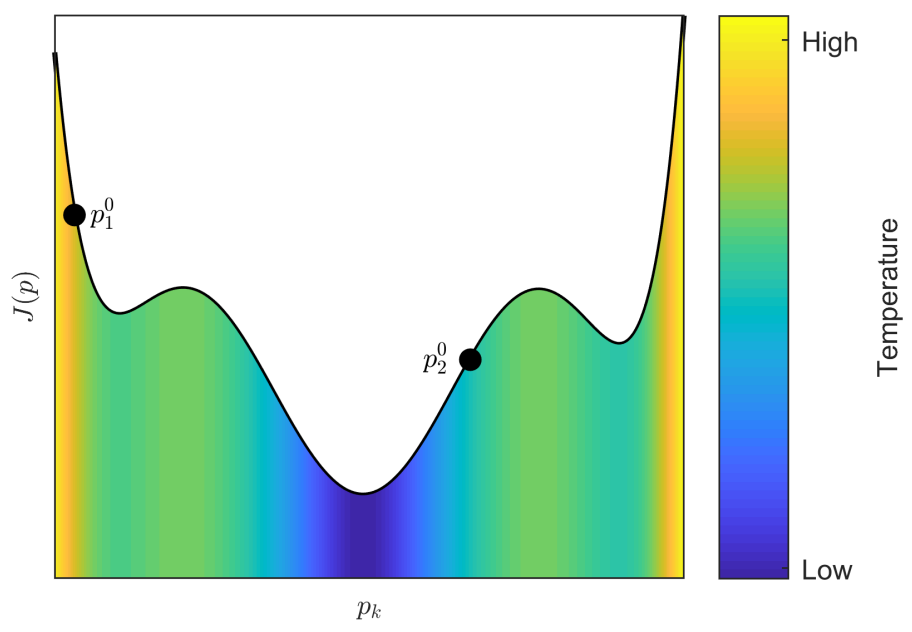

Figure 4.7: Illustration of the Simulated Annealing optimization in the likelihood landscape, as a function of temperature. The color scheme indicates the minimal temperature required for an accepted move/jump to that region of the parameter space during optimization. As seen, a high temperature (yellow) means that the optimization algorithm can climb far uphill, traversing large portions of the parameter space in the initial phase of the optimization, locating potential local minima. With decreasing temperature (green to blue color), the search is concentrated on favorable parameter regions (low $J(p)$ ). The initial parameter points $p_{1}^{0}$ and $p_{2}^{0}$ acts as potential starting points for the parameter search. Figure inspired by the work of [39]. 
Figure 4.7 depicts a simple illustration of the SA search pattern. The algorithm is initiated in a starting point $\left(p_{1}^{0}\right.$ or $\left.p_{2}^{0}\right)$ and with a high $T$ (yellow). The color depicts the minimal temperature required to accept the move to that portion of the parameter space. As seen, in the initial phase of the search, the algorithm can search the whole parameter space, making this the global search phase. As the temperature is iteratively reduced, the probability of accepting an uphill move is decreased $[188,189]$, concentrating the search in potential local/global minima. These search patterns are combined with the generation of new candidate parameter points through an user-defined function, for instance the Nelder-Mead simplex method [184, 194].

\section{Scatter search}

Scatter Search is a metaheuristic evolutionary algorithm that was first introduced by Glover in 1977 [190, 191]. Evolutionary algorithms are based on the process of evolution observed in biology, incorporating elements such as mutations, cross-breeding, and generations to evolve an initial seed of parameter candidates. Specifically, Scatter Search revolves around the use of a reference set of parameter candidates, which is iteratively updated throughout the optimization.

Figure 4.8 illustrates the basic steps underlying the Scatter Search algorithm. Scatter Search is initialized with a set of diverse initial starting points ( $p^{0}$, gray circles), which are subsequently further improved upon using a userdefined improvement method. This forms the basis of the reference set for the first iteration (blue circles, top right). This reference set is used to generate new candidate parameter points (subset) by for instance a linear combination of two or more reference solutions (dark gray circles, bottom right). These subset candidates are further improved upon and added back to the reference set. This forms an iterative loop, which stops when the termination criteria are fulfilled, typically a max number of iterations or a user specified duration. The improvement method is typically a local optimization algorithm, making Scatter Search a hybrid approach (combining both global and local search phases). This algorithm has been shown to perform well on both small and large kinetic models, especially in combination with the precise calculation of the likelihood gradient $[184,186]$.

\subsection{Assessing goodness of fit using statistical tests}

In the previous section, the topic of optimization and finding the parameters $\hat{p}$ which minimize the discrepancy between observed experimental data and model simulations was covered. We now want to test if the discrepancies between model and data are small enough, i.e., assess the goodness of fit in order to determine whether to accept or reject the model [39]. This is 


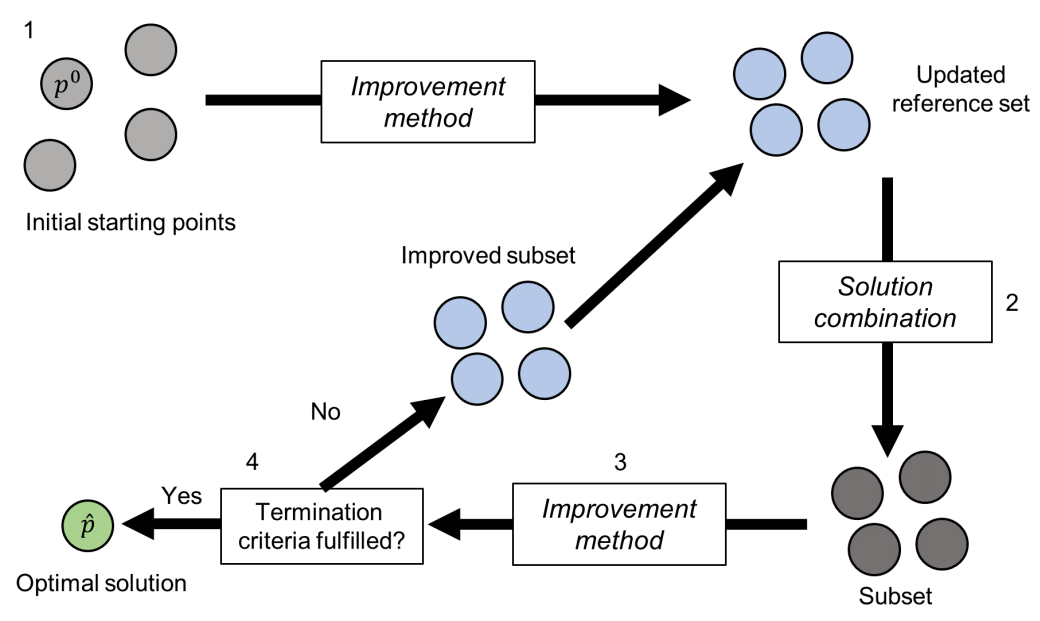

Figure 4.8: General flowchart of the Scatter Search algorithm. (1) Generate initial starting points (light gray dots), which are subsequently optimized using the chosen improvement method. These points constitute the initial reference set (light blue dots). (2) Create a subset of candidate parameter points by a linear combination of the reference set points (dark gray dots). (3) Improve the subset points through optimization. (4) Check termination criteria. If not fulfilled: update the reference set with some of the favorable subset points and repeat from (2).

formally done by evaluating the null hypothesis, $H_{0}$, which tests if the given model structure $M(\hat{p})$, with its associated error model for the observables, has generated the experimental data.

Throughout the included works in this thesis, the $\chi^{2}$-test has been extensively used. The $\chi^{2}$-test is a statistical test for the size of the model residuals, introduced by Karl Pearson in 1900 [195, 196]. Given normally distributed noise, the sum of squares of $N$ independent standard normally distributed variables (denoted $y_{i}$ ) follows a $\chi^{2}$-distribution with degrees of freedom equal to $N$ :

$$
\sum_{i=1}^{N} y_{i}^{2} \sim F_{\chi^{2}}
$$

One can note that in the case of normally distributed noise, the residuals calculated in Eq. 4.8 follow by definition a $\chi^{2}$-distribution [171, 197], and therefore the summarized cost value of Eq. 4.8 follow the $\chi^{2}$-distribution. In this case, the test statistic of the $\chi^{2}$-test, $T_{\chi^{2}}$, is equivalent to the summarized cost value, $T_{\chi^{2}}^{M}$. As such, we can now determine whether to reject the model $M$ or not by comparing the model-based test static value $T_{\chi^{2}}^{M}$ with a cut-off value $T_{\chi^{2}}^{c u t-o f f}$. This cut-off value is given by the inverse of the $\chi^{2}$ cumulative density function, with significance level $\alpha$ and $d f$ degrees of freedom $\left(F_{\chi^{2}}^{c d f-i n v}\right)$ 
[39].

$$
\begin{array}{cc}
T_{\chi^{2}}^{c u t-o f f} & =F_{\chi^{2}}^{c d f-i n v}(1-\alpha, d f) \\
T_{\chi^{2}}^{M} & =J_{s s r}(\hat{p}) \\
\text { if } T_{\chi^{2}}^{M}>T_{\chi^{2}}^{c u t-o f f}, \quad \text { reject } M(\hat{p})
\end{array}
$$

where $M(\hat{p})$ is the model structure $M$ evaluated for the optimal parameters $\hat{p}$. The significance level $\alpha$ is often chosen beforehand, but the degrees of freedom $d f$ needs to be determined. In the case of testing the model simulations on independent data (see Section 4.8), $d f$ are simply the total number of validation data points. However, one often tests the model on the same set of data that is was trained on, meaning that the residuals are not independent [198]. Nevertheless, this can be compensated for in multiple ways. The most theoretically sound way is to subtract the number of identifiable parameters from the total number of experimental data points $\left(n_{d}\right)$ :

$$
d f=n_{d}-q
$$

where $q$ is the number of identifiable parameters. A parameter $p$ is deemed identifiable if one can draw a bounded range in the parameter space, in which the parameter must lie (in order to pass a $\chi^{2}$-test, Eq. 4.18). If such a bounded range does not exist, the parameter is deemed unidentifiable. This topic is covered in detail in Section 4.7, along with other alternative ways of compensating for the lack of independence. Nevertheless, a common approach is to not compensate at all $\left(d f=n_{d}\right)$, making model validation a crucial and essential step (see Section 4.8).

\subsection{Identifiability of parameters and uncertainty analysis}

Biological systems are inherently complex systems that pose difficult challenges in the measurement domain, especially in vivo, meaning that often few properties can be measured. Furthermore, most available observations are measured indirectly or under specific experimental conditions, limiting the applicability and quality of the observations. Together, these limitations mean that all parameters in a model might not be uniquely determined from the experimental data. Such parameters are referred to as unidentifiable, and can be detected by for instance using Lie algebra as presented by Sedoglavic [199]. In practice, the uncertainty of a parameter is given as an interval of values, which might be or not be limited. If the interval is not limited in any (positive or negative) direction, the parameter is commonly denoted as structurally unidentifiable. An intuitive example is if we would measure some quantity $y^{*}$ which we postulate could be described by a relationship that is 
dependent on two parameters $p_{1}, p_{2}$ and the variable $x$ so that:

$$
y^{*}=p_{1} x-p_{2} x
$$

Using the algorithm presented in Sedoglavic [199], the parameters $p_{1}$ and $p_{2}$ would be identified as structurally unidentifiable. The reason for this is that the two decay terms in Eq. 4.20 are described in the exact same way, making it impossible to distinguish the two decay reactions from each other. In this case, the individual values of $p_{1}$ and $p_{2}$ are not uniquely determined, as an increase in one parameter can be fully compensated by a corresponding decrease of the other. Such a scenario can be remedied by simply introducing a new parameter $p_{3}=p_{1}+p_{2}$, which instead would be fully identifiable, meaning that we can identify an unique value for $p_{3}$ that satisfy the relationship. Nevertheless, given a common assumption that all parameters should be positive $(p>0)$, implying irreversible reactions, the parameters $p_{1}, p_{2}$ are now bounded in the negative direction, i.e., a lower bound of the parameters exists. In this way, the parameters are unbounded only in the positive direction. Identifying unidentifiable and identifiable parameters (given the available data) can be important for assessing the predictive power of model predictions, or to draw model-based insights regarding the biological system at hand $[171,200]$. Below, I will go through the three most common approaches for determining the identifiability of parameters.

\section{Fisher information matrix}

Historically, the oldest approach for estimating a lower and upper bound for a parameter is to use the Fisher Information Matrix (FIM) [173, 201], which is based on the Hessian or approximations thereof:

$$
F I M_{a, b}=\left.\sum_{i=1}^{n_{d}} \sigma_{i} \frac{d \hat{y}_{i}}{d p_{a}} \frac{d \hat{y}_{i}}{d p_{b}}\right|_{\hat{p}}
$$

where $a=b=1, . ., k$ and $k$ is the number of parameters; $n_{d}$ is the number of experimental time points; where $\sigma_{i}$ is the variance of each experimental data point and where $\hat{y}_{i}$ is the model simulation of the observable at time point $i$ using the optimal parameters $\hat{p}$. A confidence interval for each parameter can then be drawn as [202]:

$$
C I\left(\hat{p}_{i}\right)=\hat{p}_{i} \mp T_{t}(\alpha, d f) \hat{\sigma} \sqrt{F I M_{i, i}}
$$

where $T_{t}(\alpha, d f)$ is the student's t-test for confidence level $\alpha$ and $d f$ degrees of freedom, and where $\hat{\sigma}$ is the true variance of measurement data $[173,202]$.

The confidence intervals generated from the FIM rely on two key assumptions: 1) that the likelihood landscape in the neighborhood of the local/global 
minima is described by an ellipse, implying that all parameters are identifiable (hence you need to assume identifiability to prove identifiability) [198, 202], and 2) that the observations depend linearly on the model parameters. For complex non-linear models, these assumptions might not be valid to make $[202,203]$. Due to these limitations, other methods which do not rely on such assumptions have been developed.

\section{Profile likelihood}

Profile Likelihood (PL) determines profiles for each individual parameter separately. Using PL, the parameter $p_{k}$ is traced along its numerical axis, for both positive and negative directions, allowing every other parameter pertaining to the model to change $\left(\tilde{p}_{j \neq k}\right)$ for each value of $p_{k}$. This is typically expressed as:

$$
\mathrm{PL}_{k}\left(p_{k}\right)=\min _{\tilde{p}_{j \neq k}} J\left(\tilde{p} \mid \tilde{p}_{k}=p_{k}\right)
$$

A PL analysis approximates the optimal path through the parameter space for each $p_{k}$, which describes the curvature of the likelihood function $J(p)$ on a global scale, as well as determining the feasible range of values for the parameter [200, 204]. The identifiability of the parameter $p_{k}$ is determined by a threshold, specified as:

$$
D:=\operatorname{PL}\left(p_{k}\right)-J(\hat{p}) \leq \chi_{\alpha, d f}^{2}
$$

where $\chi_{\alpha, d f}^{2}$ is the $\alpha$ quantile of the $\chi^{2}$ distribution for $d f$ degrees of freedom. The choice of $d f$ is non-trivial, and is often set as: 1) $d f=1$, which is the theoretically correct value, yielding a narrow confidence interval, but does not account for the uncertainty present in experimental data; 2) $d f=n_{p}-n_{i}$, where $n_{p}$ is the number of parameters and $n_{i}$ is the number of identifiable parameters (typically not known a priori), or 3 ) $d f=n_{p}$, correcting for multiplecomparisons and giving simultaneous confidence intervals for all parameters. An additional common way of specifying the PL threshold is to use the $T_{\chi^{2}}^{M}$ as the absolute upper threshold, meaning that each point of the profile must remain in statistically significant agreement with the experimental data. In practice, the profile likelihoods are calculated using repeated-optimization $[198,205]$ or by using an integration-based approach as described in [206208].

In Figure 4.9, three cases of identifiability are shown, calculated using a PL analysis. The top row (Fig. 4.9A, C, E) depicts the likelihood landscape contours as dependent on two parameters $\left(p_{1}, p_{2}\right)$, while the bottom row (Fig. $4.9 \mathrm{~B}, \mathrm{D}, \mathrm{F})$ shows the trace of $p_{1}$ with the associated change in model agreement to data. The $\chi^{2}$-threshold for $d f=1$ is shown as thick solid lines in all graphs. In the left column, the trace of the profile is flat (Fig. 4.9B), thus failing to establish both an upper and lower bound of $p_{1}$. This case for a PL 
analysis of the same nature as Eq. 4.20, i.e., structural unidentifiability. This particular issue cannot be resolved by increasing the quality of measurement data, but requires either reformulation of the model structure or new measurement observables. In the middle column (Fig. 4.9C, D), a lower bound of $p_{1}$ can be established, but not an upper bound (Fig. 4.9D). This case is commonly referred to as practical unidentifiability, where the trace is only limited in one direction. This case can sometimes be resolved given higher quality data, meaning less measurement noise, which would essentially move the $\chi^{2}$ cut-off threshold in a downwards direction. However, the addition of higher quality data does not guarantee that the parameter will become identifiable. The final case is presented in the right column, where $p_{1}$ exhibit both an upper and lower bound (Fig. 4.9F), which means that the parameter is identifiable as there exist a limited range of values that the parameter must reside within for the model to agree with data.
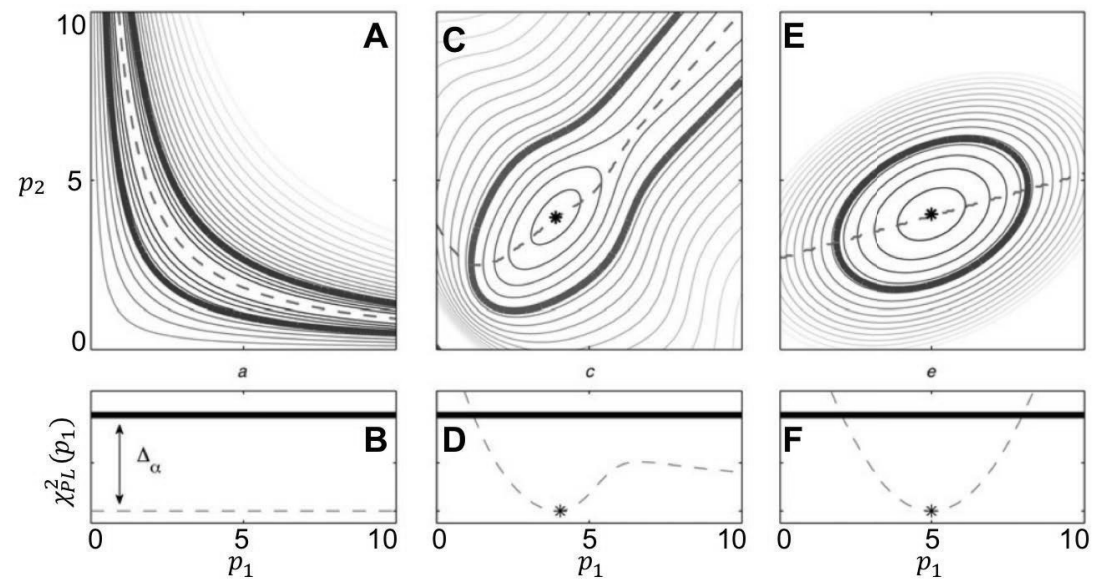

Figure 4.9: The three cases of parameter identifiability and the profile likelihood. Top row: contour plots of the three different likelihood landscapes (solid lines) as dependent on two parameters $\left(p_{1}, p_{2}\right)$. The $\chi^{2}$-threshold is highlighted as thick black lines in both top and bottom row graphs. The dashed lines correspond to the profile likelihood trace. Bottom row: the profile likelihood trace of $p_{1}$ along with the associated change in the residual cost. A, B depicts structural unidentifiability, as the residual cost does not change along the trace of the profile. C, D depict practical unidentifiability, where only a lower boundary of $p_{1}$ can be established. Lastly, E, F depict the final case where the parameter is identifiable, as there exist a lower and upper bound. Originally published in [209]. Included with the permission of the authors.

Re-examining the example provided in Eq. 4.20, this case would for a PL analysis be considered identifiable. This is due to the fact that a lower bound (given the assumption $p>0$ ) exists, and an upper bound can be calculated with respect to data, given by the fact that $p_{3}=p_{1}+p_{2}$ is identifiable (see example and discussion in [198]). For these reasons, the definition of structural 
unidentifiability is dependent on the analysis method used, and the classical analysis of structural unidentifiability (such as in [199]) does not rule out that the parameter could be in practice identifiable (for instance using a PL analysis). This means that the classical way of denoting structural unidentifiability is of lesser importance in terms of identifiability of the model parameters.

\section{Markov Chain Monte Carlo (MCMC) methods}

An alternative to a PL analysis is to assess the posterior distributions of the parameters, which is typically performed using Markov Chain Monte Carlo (MCMC) sampling. The posterior distribution is defined in accordance to Bayes' theorem as:

$$
P(p \mid y)=\frac{L(y \mid p) P(p)}{P(y)}
$$

where $P(p \mid y)$ represents the probability of observing the parameters $p$ given the measurement data $y$; where $L(y \mid p)$ is the likelihood function maximizing the probability of observing data given $p$; where $P(p)$ is the prior belief or knowledge about the parameter values, and finally, where $P(y)$ is a scaling factor, which is not known a priori but can for parameter inference purposes be ignored as it is independent of $p$. In analogy to the likelihood function, a maximum a posteriori estimate is gained by maximizing $P(p \mid y)$ which is equivalent to minimizing $J(p)$ or maximizing $L(y \mid p)$. If the prior belief $(P(p))$ is an uniform distribution on the interval given by the parameter boundaries, the posterior distribution corresponds to the likelihood as subjected to the prior. If the prior is not known, the prior distribution can be flat, meaning an equal probability for all possible parameter values. By repeated sampling using various MCMC algorithms, the posterior distribution will converge to the true target distribution asymptotically, given enough iterations.

The most widely known algorithm is the Metropolis-Hastings algorithm [210, 211]. It samples the posterior distribution of the parameters by performing random walks throughout parameter space. At every parameter point $p$, a candidate point $p^{\prime}$ is drawn from a proposal distribution $q(p) . q(p)$ is typically a normal distribution: $q(p)=N(p, \Sigma)$ with the covariance matrix $\Sigma$. The most common example of the algorithm is simply a normal distribution $N(p, 1)$. The candidate point is accepted with the probability $\alpha\left(p^{\prime}, p\right)$ :

$$
\alpha\left(p^{\prime}, p\right)=\min (\underbrace{\frac{L\left(y \mid p^{\prime}\right) P\left(p^{\prime}\right)}{L(y \mid p) P(p)}}_{\text {posterior ratio }} \cdot \underbrace{\frac{q\left(p \mid p^{\prime}\right)}{q\left(p^{\prime} \mid p\right)}}_{\text {Hastings ratio }}, 1)
$$

This expression contains the likelihood ratio between the candidate and current parameter point, as well as the Hastings ratio, which ensures that the 
sampler can reverse its previous move in space. This means that the sampler does not get stuck in one site. If the candidate point is more probable than the existing point, meaning that the posterior ratio is $>1$, the candidate point is accepted. However, if the candidate point is less probable, the point is sometimes rejected, with the likelihood of rejection increasing the less probable the candidate point is. Therefore, with many iterations (samples), the MCMC sampling algorithm will visit high probability regions many times, while rarely visiting low probability regions. This forms the posterior distribution of the parameters. MCMC methods have continued to evolve, and more advanced algorithms now exist. For instance, algorithms which include adaptation of the proposal distribution $q$ such as the adaptive Metropolis algorithm [212]; incorporates the local geometry of the likelihood landscape such as in the manifold Metropolis adjusted Langevin algorithm, or multi Markov chain approaches such parallel tempering [213, 214].

Figure 4.10 show the similarity in parameter identifiability between an MCMC approach, the Region-Based Adaptive PARallel Tempering approach introduced by [214], and the conventional repeated-optimization PL analysis (Eq. 4.23). The test case consists of a pharmacokinetic model describing the hepatic uptake and degradation of an MR-compatible liver contrast agent, with MRI-based measurements of the amount of contrast in the liver and spleen compartments [215]. The model consists of five parameters. As seen, the PL traces for each parameter (Fig. 4.10, black lines) crosses the $\chi^{2}$-threshold $(\alpha=0.05, d f=1)$ at similar parameter values as the MCMC based posterior distributions of the parameters (Fig. 4.10, gray samples). The global optimization convergence points are shown as red dots, overlapping with the peaks of the posterior distributions. Nevertheless, two parameters: $k_{h p}$ (top left) and $k_{h b}$ (bottom left) are practically unidentifiable which both methods show: PL as the black line never crosses the $\chi^{2}$-threshold, and MCMC by having samples (albeit with low probability), in the non-limited direction. The graphs are truncated to -6 in $\log _{10}$ space for visual clarity.

While a PL analysis is performed on a per parameter basis, an MCMC analysis instead generates the posterior distributions for all parameters at once. As PL is computationally heavy to perform due to the conventional repeatedoptimization approach, it becomes computational cumbersome for highly parameterized models. In such cases, MCMC might be a better choice but requires large amounts of samples in order to converge to the true posterior distribution. In this thesis, both PL and MCMC approaches has been extensively used. The simulation uncertainties in Paper III and IV are based on an MCMC approach, while key results in Paper II are based on the use of PL analysis. 


\section{Parameter identifiability}
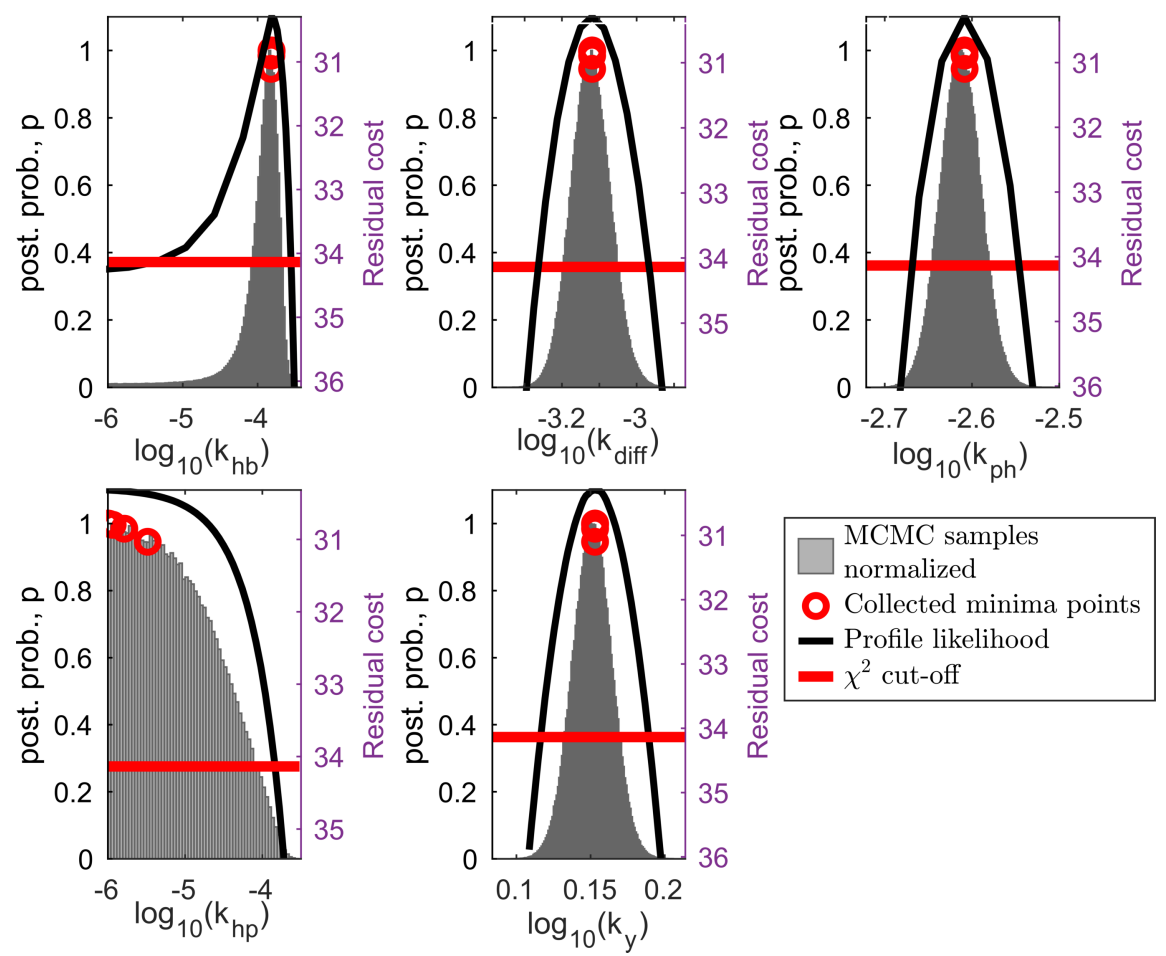

Figure 4.10: Comparison between MCMC and PL for a five parameter model of liver contrast agent uptake. Normalized MCMC samples are shown as the gray histograms, while the profile likelihood trace is shown as the solid black lines. The $\chi^{2}$-threshold for $d f=1$ is shown by the horizontal red line. Three parameters are identifiable $\left(k_{d i f f}, k_{p h}, k_{y}\right)$, while two are practically unidentifiable $\left(k_{h p}, k_{h b}\right)$.

\section{Generalizing parameter uncertainty to prediction uncertainty}

The underlying methods behind both PL and MCMC can be extended from determining the uncertainty of parameters to any specified model property, $Z(p)$, that can be quantified from the model simulations [198, 200]. For PL, this is commonly denoted as the prediction profile likelihood (PPL), and can be estimated using the repeated-optimization method with a modified likelihood function:

$$
J(p)_{\mathrm{PPL}}=J(p)+\lambda\left|Z(p)-Z^{\prime}\right|
$$

where $J(p)$ is the residual cost function; where $\lambda$ is the L1-weight, chosen to be sufficiently large to ensure that the added term is heavily favored to be minimized over $J(p)$; where $Z(p)$ is the model simulated property value for the given parameters $p$, and finally, where $Z^{\prime}$ is the fixated value to be reached for the model property, which is iteratively changed. This technique is used 
in Paper II to look at the ratio between glutamate and GABA release, and to calculate the simulation uncertainty against estimation and validation data.

\subsection{The necessity of validation data}

We have now reached step 3 in the modeling cycle depicted in Figure 4.1, where model-based predictions are to generated and used for two purposes: 1) validating the model by comparing the model-based predictions to independent validation data, or 2) suggest new experimental designs for further investigation of both the biological system, and validation of the model structure. Using the methods described in Section 4.7, we now know how to generate model predictions with an upper and lower bound of uncertainty. However, why use validation data at all?

\section{Model over-fitting and selection}

Over-fitting a model occurs when the model contains too many parameters (i.e., over-parameterized) as compared to both the quality and quantity of measurement data for which the parameters have been estimated on [171]. This is typically the case in Systems Biology, where available experimental data is usually limited and the model complexity is large due to the previous knowledge about the system. Over-fitting is often suspected if the model simulation fits the estimation data points too well, for instance fitting outlying data points or following the measurement noise too closely. If a model is over-fitted, the typical tell-tale sign is that it will not be able to predict independent validation data.

A paper by Sundqvist et al. [216] illustrates this common occurrence within Systems Biology. A polynomial example is presented in Figure 4.11. Here, simulated data (Fig. 4.11A-C, blue and red error-bars) have been generated using a $7^{\text {th }}$-degree polynomial as the ground truth, and corrupted using additive white noise of three different levels. For high measurement noise, a simple linear model is sufficient to describe the estimation data (Fig. 4.11A, blue symbols), but will fail to predict the validation data (Fig. 4.11A, red symbols). If the measurement noise is small, the polynomial degree must be high in order to fit the estimation data (Fig. 4.11C). Nevertheless, the added complexity to fit estimation data warps the ability to accurately predict the validation data (Fig. 4.11C, red symbols). Therefore, the correct model structure has to both be able to capture the dynamics in estimation data, but also correctly predict validation data, as quantified by having a low SSR (Eq. 4.8) against data used for validation (Fig. 4.11B).

To further illustrate this point, 14 different model structures, ranging from a first order to a 14th order polynomial expression, were fitted to the simu- 

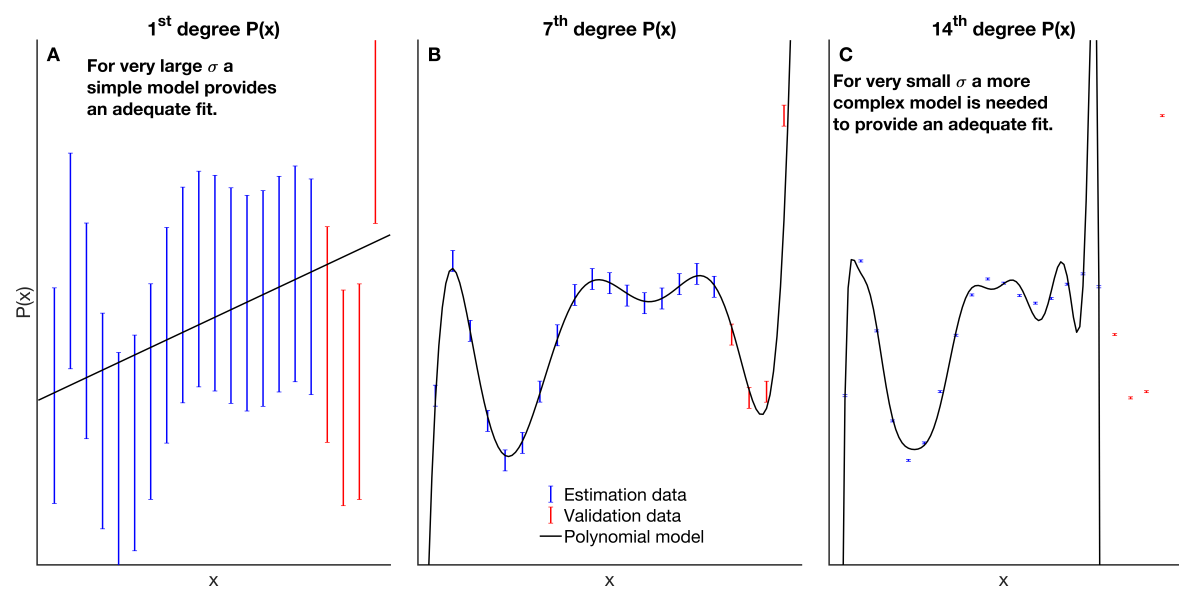

Figure 4.11: Simple example using three polynomials. For the three cases: synthetic data (red and blue symbols) generated by a 7th order polynomial (B, black line), plus some normal additive noise of three different levels. Using the $\chi^{2}$-test, high noise favors a simple model (A, black line), and low noise favors a complex model (C, black line). Nevertheless, these incorrect model structures have less predictive power of data used for validation (red symbols), unlike the true model structure (B). Image taken from [216] with permission from the authors.

lated noise corrupted data of 11 different levels. Model selection was carried out using four different methods:

1. Select the model structure with the lowest SSR (Eq. 4.8).

2. Select the model structure with the lowest $\chi^{2}$-test statistic, $T_{\chi^{2}}^{M}$, with the degrees of freedom corrected for number of parameter $d f=n_{d}-n_{p}$ where $n_{d}$ is the number of data points and $n_{p}$ is the number of parameters.

3. Select the simplest model structure that pass the $\chi^{2}$-threshold, corrected for as in method 2.

4. Select the model with best prediction of the validation data (determined by the lowest SSR).

The model selection results are shown in Figure 4.12 with the left column depicting method 1) and 2), middle column depicting method 3), and finally, right column depicting method 4). The estimation SSR for the 11 different noise levels (blue, green, red lines) is shown for the different methods (Fig. 4.12A-C). The selected model is indicated by the colored dot for each noise level. The bottom row (Fig. 4.12D-F) depicts the model selection against the noise level. As seen, the first method of selecting the model with the lowest 

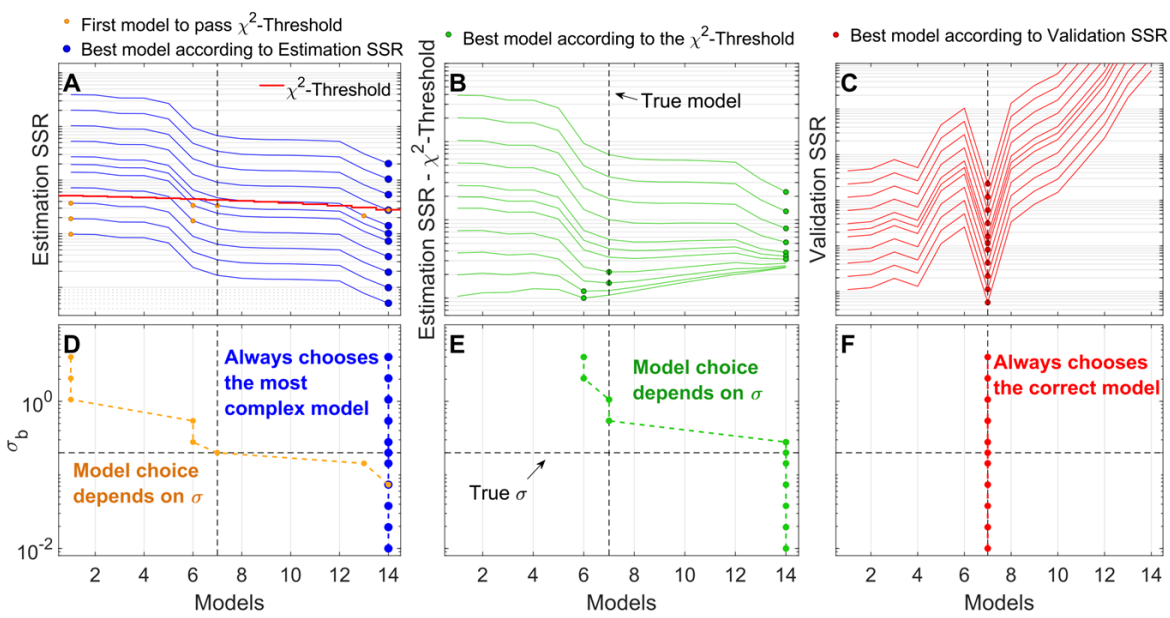

Figure 4.12: Polynomial example of model selection using four different methods. For each graph: models with increasing complexity (1-14) on the x-axis, residual cost for estimation data $(\mathbf{A}-\mathbf{C})$ or the noise level of data $(\mathbf{D}-\mathbf{F})$ on the y-axis. A-C: Results for method 1 ('Lowest SSR') and 2 ('Simplest $\chi^{2}$ ') (A); method 3 ('lowest $\chi^{2}$ '), (B), and finally, method 4 ('lowest validation SSR'), (C). The different lines (blue, green, red) corresponds to different noise levels. Method 1 always chooses the most complex model (blue dots), while method 2 choose the simplest model that pass the $\chi^{2}$-threshold (yellow dots). The selection of method 3 is dependent on the noise level (green dots), while method 4 always selects the correct model structure (red dots). D-F: Same results as in A-C, but with measurement noise versus selected model structure depicted. Method 1 is biased towards the most complex model, model selection by method 2 $\& 3$ is noise dependent, and method 4 always chooses the correct model structure, independent of the level of measurement noise. Image taken from [216] with permission from the authors.

SSR will always choose the most complex model (Fig. 4.12A, D). For the $\chi^{2}-$ based model selections (method $2 \& 3$ ), the model selection depends on the measurement noise, and the correct model structure is rarely selected (Fig. 4.12A, D; B, E). For the last method (4), which uses validation data, the correct model structure is always chosen, independent of measurement noise (Fig. 4.12C, F).

Therefore, using independent validation data is crucial to validate models within Systems Biology. Furthermore, model selection using validation data is noise independent, favoring the correct model structure over too complex or too simple models.

\subsection{Summary}

In this chapter, an overview of the central aspects and methods within mathematical modeling has been presented. Mathematical modeling within biology has emerged as a powerful tool for studying many different diseases, organs, and biological systems, including the NVC (see Section 2.3 for an overview 
of existing models of the NVC). This chapter illustrates the iterative cycle of mathematical modeling, as seen in Fig. 4.1), and covers many of the commonly used methods behind each of the steps involved. These methods and tools can now be applied to the question and aim outlined in Section 2.4: to develop a mechanistic model of the NVC. 


\section{5}

\section{Results}

In this chapter, I present the main findings of my thesis. The results are presented in the natural order: i.e., for Paper I-IV.

\subsection{Paper I: evaluating two of the most widely discussed hypotheses underpinning the NVC}

\section{A Metabolic feedback hypothesis}

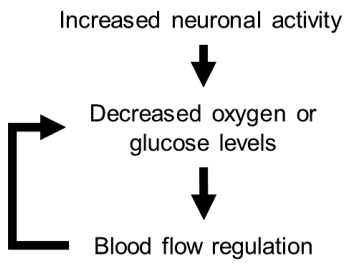

Reactive system

\section{B \\ Feed-forward hypothesis}

Increased neuronal activity

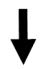

Intracellular signaling

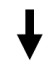

Relase of vasoactive messengers

Proactive system

Figure 5.1: The two studied hypotheses in Paper I. A: The metabolic feedback hypothesis. Neuronal activity consumes energy in the form of glucose and oxygen, which triggers an increase in blood flow through a feedback loop. B: The feed-forward hypothesis. Neuronal activity triggers intracellular signaling cascades, culminating in the release of vasoactive messengers which act on the vasculature. Image adapted from Figure 1 in Paper 1.

In Paper I, we evaluated the two most widely discussed hypotheses describing NVC: the metabolic feedback hypothesis (see Section 3.1, Fig. 5.1A) and the feed-forward hypothesis (see Section 3.2, Fig. 5.1B), using BOLDfMRI data consisting of two visual stimulation paradigms (see Paper 1, Experimental data). The two paradigms included an intensity aspect using different contrast levels of the visual cue, and a frequency aspect with a pair of 
visual cues presented within a short time interval. The models were evaluated in regards to the ability to produce the qualitative features of the BOLD response: an initial dip, a main overshoot, and a post-peak undershoot (Fig. 2.6A), as well as the quantitative agreement with experimental data.

\section{Neither hypothesis alone can explain all three characteristics individually}

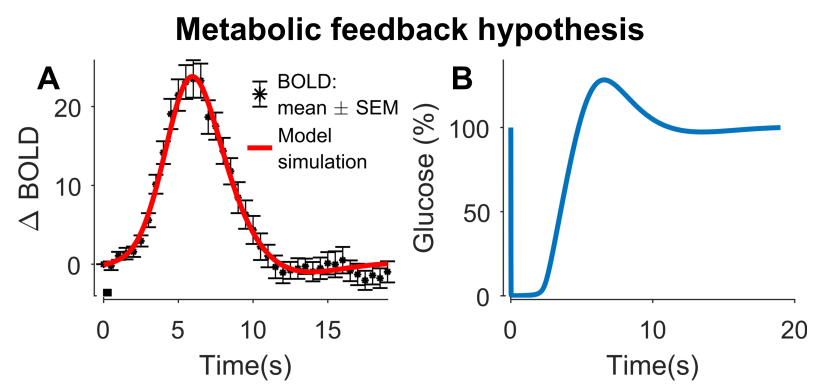

Forcing an initial dip
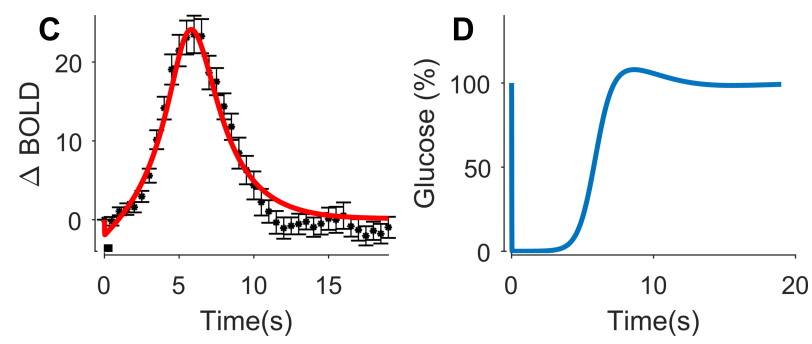

Figure 5.2: The rejection of the metabolic feedback hypothesis. Model simulation of the BOLD response $(\mathbf{A}, \mathbf{C})$ and glucose $(\mathbf{B}, \mathbf{D})$ for an unconstrained scenario $(\mathbf{A}, \mathbf{B})$, and a forced initial dip scenario (C,D). For each graph: model simulation (colored line), BOLD data (black symbols, with error bars depicting standard error of the mean (SEM)), and finally the length of stimulation ( $500 \mathrm{~ms}$ ) is indicated by the black bar in the lower-left portion of $\mathbf{A}, \mathbf{C}$.

Following the modeling cycle outlined in Figure 4.1, multiple model structures capturing the main mechanisms of the metabolic feedback hypothesis was constructed. More specifically, for one of the model structures (see Fig. 5.1A; Fig. $5 \mathrm{~F}$ in Paper I), glucose acts as the regulator in a negative feedback system. Thus, when glucose levels decrease upon stimulation, a corresponding increase in blood flow is evoked as a counteracting force. As can be seen, while the model can quantitatively fit the BOLD-fMRI data, it can not capture all of the qualitative features simultaneously. In Fig. 5.2A, the model does not produce an initial dip, and if an initial dip is forced as in Fig. 5.2C, the ability to produce a post-peak undershoot is diminished. Furthermore, in order to quantitatively describe the BOLD data, the model resorts to biologically implausible glucose behavior (Fig. 5.2B, D). Upon stimulation, the model glucose level is reduced to almost zero, $(<1 \%$ of the baseline value), 
and remains there for several seconds. In a similar way, all other variants of a metabolic feedback system, including using oxygen level as the regulator, could not pass the hypothesis testing (see Paper I). In contrast, a model structure describing the feed-forward hypothesis could simulate all three BOLD characteristics and quantitatively agree with experimental data (see Figure 6 in Paper I). However, model analysis revealed that it could only do so in an implausible way, as the initial dip in the model was caused by rapid vasoconstriction before the overshoot phase of the response, which is not in line with previous insights and data (see discussion in $[125,126]$ ). For this reason, the feed-forward hypothesis was rejected.

\section{A combined model can quantitatively describe and qualitatively predict data}

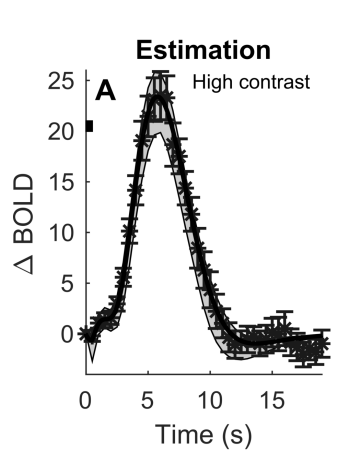

Intensity experiment
Validation
$25+$ Low contrast
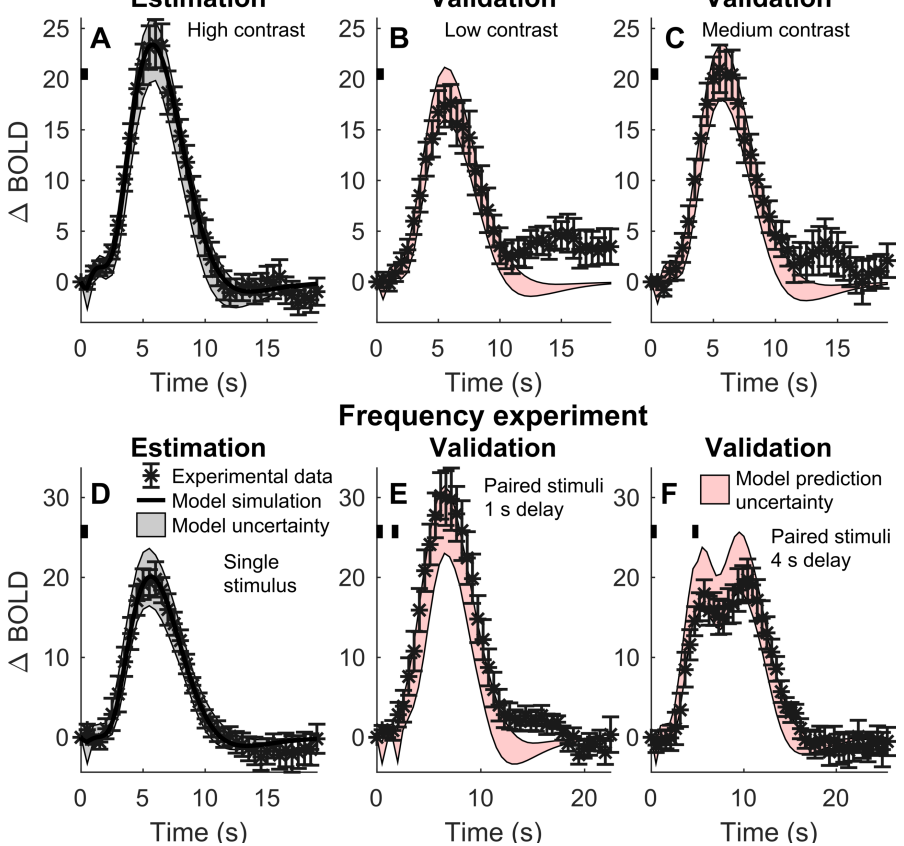

Frequency experiment

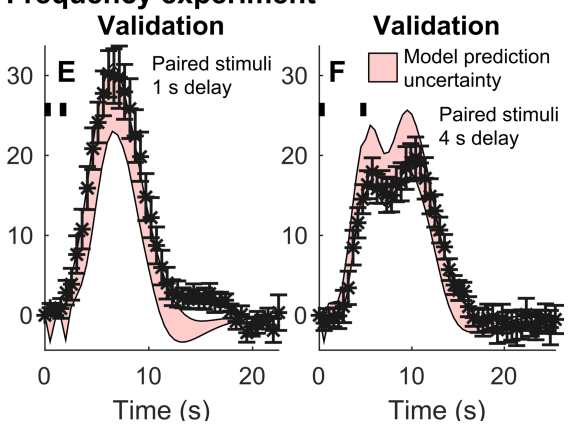

Figure 5.3: A combined model can describe both data used for estimation and predict independent validation data. A, D: Model estimation to data from the intensity (A) and frequency (D) experiment. B-C, E-F: Model predictions compared to validation data for the intensity (low (B) and medium (C) contrast) and frequency (two stimulations, $1 \mathrm{~s}(\mathbf{E})$ and $4 \mathrm{~s}$ (F) delay) experiments. For each graph: the x-axis depicts time in seconds, the y-axis is the normalized BOLD response, and experimental data is shown in black symbols with error bars representing SEM. For A, D, best simulation is shown as solid colored lines, with the corresponding model uncertainty as the black shaded area. For $\mathbf{B}-\mathbf{C}, \mathbf{E}-\mathbf{F}$, the model prediction uncertainty is shown as the red shaded area. The stimulation paradigm is indicated by the black rectangle in each graph, with each stimulation being $500 \mathrm{~ms}$. Figure adapted from Paper 1. 

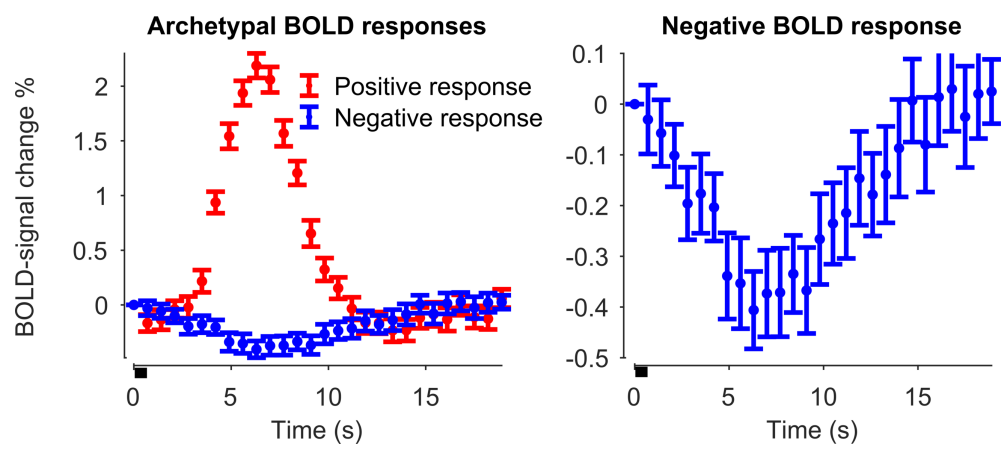

Figure 5.4: Archetypal characteristics of a positive and negative BOLD response to a brief stimulus. The positive response depicts the three main characteristics: an initial dip, an overshoot, and a post-peak undershoot. The black rectangle in the lower-left corner of each graph indicates the stimulus length of 500 ms. Right: Zoomed-in view of the negative BOLD response shown in left. As seen, the response consists of only an undershoot phase. Image adapted from Paper II.

Based on these insights generated from the two hypothesis rejections, we constructed a new model that included key aspects from both the previous hypotheses. In this combined model, the initial dip is caused by a rapidly increased oxygen metabolism triggered by the stimulation, and the overshoot and the post-peak undershoot are the result of multiple feed-forward signaling arms which release vasoactive messengers. The combined model displayed all three qualitative characteristics with a good quantitative agreement to data (Fig. 5.3A). Model predictions (red shaded areas) of data from the intensity paradigm had adequate agreement with the corresponding validation data (Fig. 5.3B-C, black symbols). The model was also fitted to experimental data from the frequency paradigm, exhibiting corresponding good qualitative and quantitative agreement to training data (Fig. 5.3D). The corresponding model predictions of the BOLD response (red shaded areas) for a paired visual cue, with $1 \mathrm{~s}$ (Fig. 5.3E) or $4 \mathrm{~s}$ (Fig. 5.3F) delay between the two visual cues, agreed well with the corresponding validation data. For more details, see Paper I.

\subsection{Paper II: negative BOLD responses - the role of inhibition}

The final model structure presented in Paper I was developed for only positive BOLD responses. Nevertheless, negative BOLD responses are commonly observed for a wide range of cognitive or clinical studies [217-220]. An archetypal negative BOLD response for a brief stimulus is depicted against a positive response in Figure. 5.4. As seen, the negative response often exhibits a shallower deflection, as compared to its positive counterpart. Furthermore, 
its qualitative features are less well defined except for the main bulk of the signal should decrease below baseline, but a brief post main response overshoot can sometimes be observed (see for instance experimental data in [221, 222]). Different hypotheses regarding the origin of the negative BOLD exists (reviewed in [223]) including hemodynamic steal [224, 225] or neuronal inhibition [221, 226, 227]. Hemodynamic steal describes how a positively activated area (in terms of BOLD) causes redistribution of blood, reducing the blood supply in the surrounding area. Neuronal inhibition instead describes how inhibitory activity of neurons reduces $\mathrm{CBF}$ and $\mathrm{CMRO}_{2}$ in that local area.

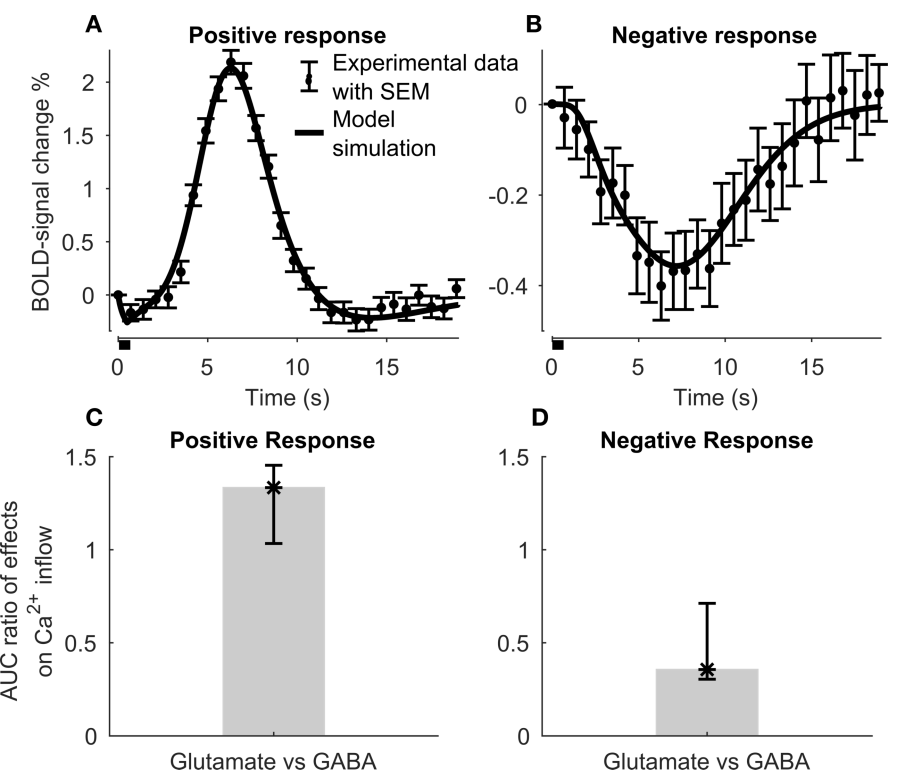

Figure 5.5: Model estimation to data consisting of brief visual stimuli evoked BOLD responses. A \& B: Experimental data (black symbols) depicting mean value and SEM for each time point compared to model simulation (black line) for both positive (A) and negative (B) BOLD responses. The $500 \mathrm{~ms}$ visual stimulation is indicated by the black rectangle in the lowerleft portion of each graph. C \& D: Area under curve (AUC) plots displaying the ratio between glutamate and GABA which have a positive or negative effect on $\mathrm{Ca}^{2+}$ influx, respectively. The uncertainties (black error bar) depict a confidence interval $(p<0.05)$ for all possible model parameters calculated using a prediction profile likelihood (PPL) approach. The figure is adapted from Fig. 4 in Paper II.

As covered in Section 2.3, many models describing the various aspects of the NVC exist. However, none of these models describe any of the hypotheses underlying the negative BOLD response. Therefore, in Paper 2, we sought to extend the model developed in Paper I to incorporate the neuronal inhibition hypothesis. The neuronal inhibition hypothesis was chosen due to the availability of such experimental data: a visual-motor task and a working-memory task which BOLD-fMRI data did not contain adjacent areas exhibiting posi- 
tive and negative BOLD responses, making the hemodynamic steal effect unlikely to be the underlying cause.

We trained this extended model using positive and negative BOLD responses acquired using the visual-motor task (see Paper II, Fig. 3). The estimated model agreed well with both the positive and negative BOLD-fMRI data, and the model simulation displayed the qualitative characteristics of both the positive (initial dip, main response, post-peak undershoot) and the negative (signal below baseline) response (Fig. 5.5A-B). Next, we quantified the ratio between the positive and negative contributions that glutamate and GABA exert on $\mathrm{Ca}^{2+}$ influx, respectively. This quantification was done using PPL analysis (see Section 4.7). The analysis revealed that the positive effect of glutamate is dominant for the positive BOLD response, as the ratio is $>1$ (model confidence interval as black error bar) for all acceptable parameter sets (Fig. 5.5C), thus increasing the vasoactive signaling. In contrast, the negative effect of GABA is instead dominant for the negative BOLD response (Fig. 5.5D), with the ratio $<1$ for all acceptable parameter sets. For more details, see Paper II.

\subsection{Paper III: assessing cell-specific contributions to the NVC and the role of anesthesia}

In Paper III, we assessed experimental data of arteriolar diameter responses captured in mice using OG stimulation of pyramidal neurons or GABAergic interneurons, as well as sensory stimuli, as presented in Uhlirova et al. [88]. These data were captured for both awake and anesthesia conditions, exhibiting qualitative and quantitative differences in-between the two conditions (Fig. 5.6A-B). Most prominently, a prolonged post-peak undershoot for the OG stimulation during anesthesia is observed (Fig. 5.6A). Currently, no existing model describes the vasoactive signaling pathways of the relevant neuronal sub-types (outlined in Fig. 5.6C) and their interplay, or include the effects of an anesthetic upon the evoked arteriolar response. We therefore sought to construct a first model describing the mechanisms and interplay between GABAergic interneurons and pyramidal neurons in the context of NVC, which include the effect of the used anesthetic. The constructed model is depicted in Figure. 5.6D, and consists of three layers: neuronal activity, intracellular signaling pathways which culminates in the release of vasoactive messengers, and finally, the vascular response.

The constructed model agrees well with data used for model estimation, which consisted of optogenetic and sensory stimuli in both awake and anesthetized mice (Fig. 5.7, compare black symbols with green lines). Furthermore, the model was used to generate predictions (Fig. 5.7, red shaded areas) of the effect of different pharmacological modulators on the evoked arteriolar 


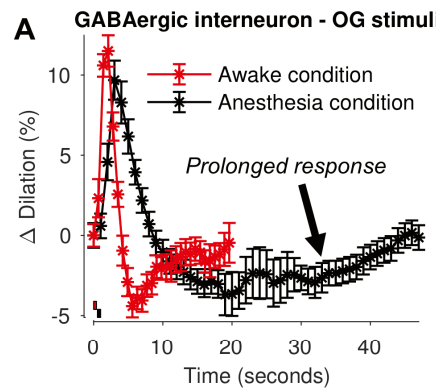

C Neurovascular coupling

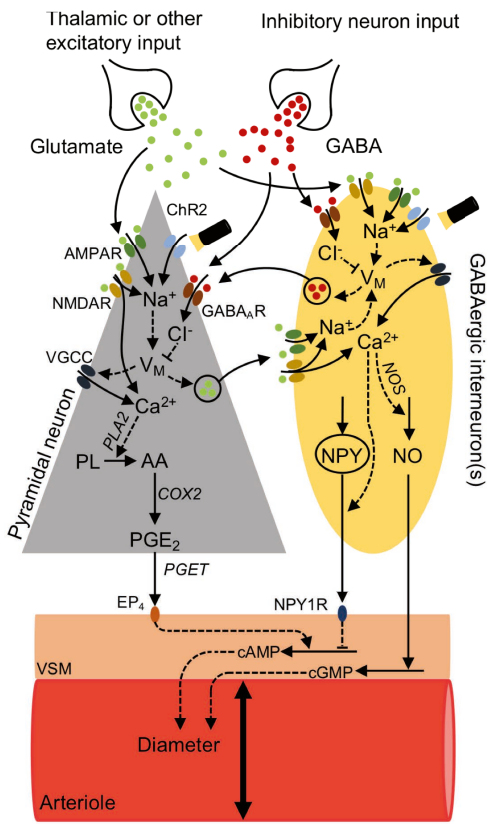

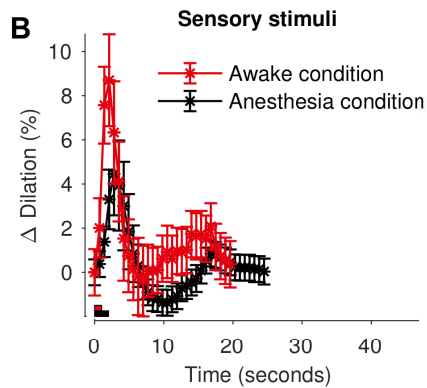

D

Model structure

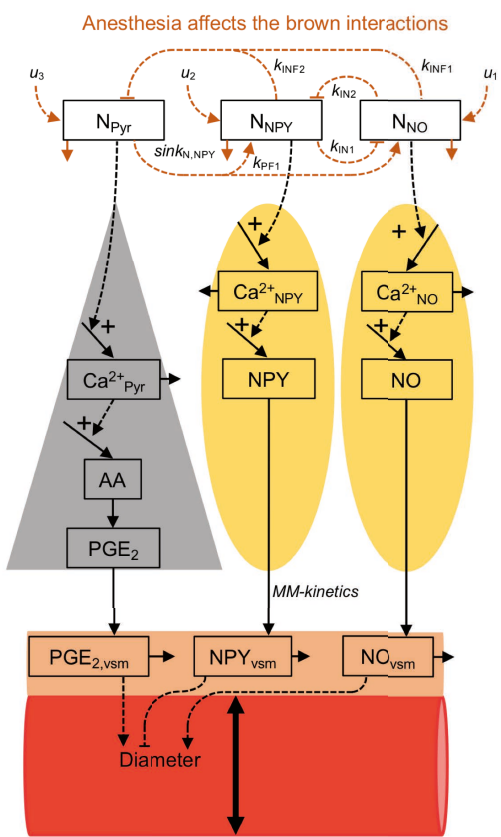

Figure 5.6: A \& B: Anesthesia alters the evoked arteriolar diameter response for both optogenetic (OG) (A) and sensory (B) stimuli. The bars in the lower-left corner of each graph indicate the stimulus length of the OG and sensory stimuli, for both awake (red) and anesthesia (black) conditions. C \& D: NVC representation of relevant signaling pathways and mechanisms (C), with the translated NVC model structure to explain these data (D). The model consists of a pyramidal neuron (gray triangle) and two GABAergic interneurons (yellow ovals), each with their respective vasoactive signaling pathways which influence the arterioles (red cylinder). See Fig. 2A-B in Paper III for further details. Figure adapted from Paper III.

response. These pharmacological modulations were: 1) the inhibition of the NPY receptor (NPY1R) for both OG and sensory stimulations during anesthesia (Fig. 5.7,E-H), and 2) the blockade of glutamatergic signaling for OG stimulation during anesthesia (Fig. 5.7I-J). These model predictions agreed well with the corresponding independent validation data (Fig. 5.7, red symbols). 


\section{Experimental design}

A

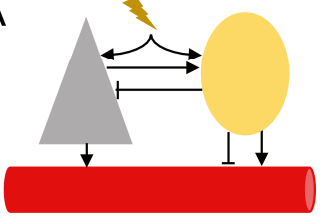

C

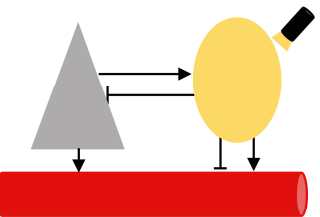

E

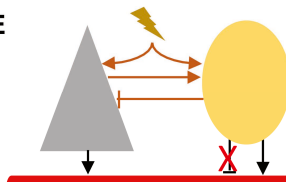

G

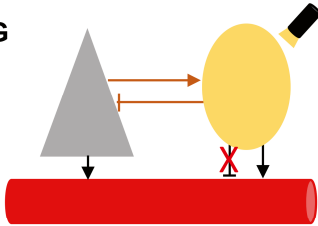

I

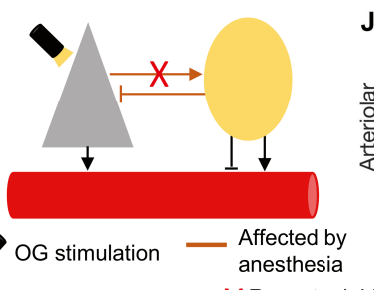

Sensory stimulation $X$ Receptor inhibitor
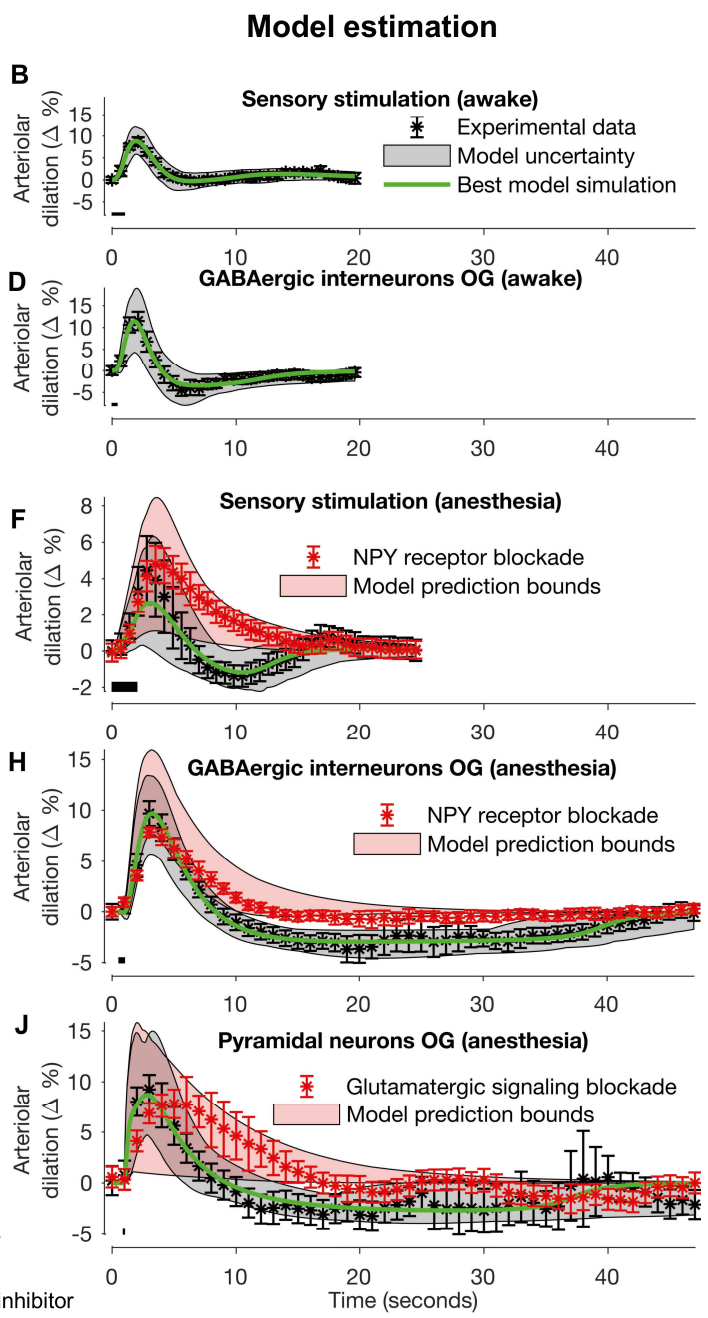

Figure 5.7: Model estimation and prediction of arteriolar diameter responses in awake (A-D) and anesthetized mice $(\mathbf{E}-\mathbf{J})$, captured using sensory $(\mathbf{A}-\mathbf{B}, \mathbf{E}-\mathbf{F})$ and optogenetic $(\mathrm{OG})(\mathbf{C}-\mathbf{D}$, G-H, I-J) stimuli. A, C, E, G, I: Simple illustrations of the experiment trial for each data set. Gray triangle: pyramidal neuron; yellow oval: GABAergic interneurons. Sensory stimulation and OG stimulation indicated by symbols, and the brown arrows are affected by anesthesia. B, D, F, H, J: The estimated model compared to experimental data. For each graph, the best model simulation (solid green line) with corresponding model uncertainty (black shaded areas), compared to experimental data (black symbols). Additionally, model predictions (red shaded areas) of different receptor inhibitors (see illustrations and legend) compared against corresponding independent validation data (red symbols). The length of the stimulation is shown by the black bar in the lower-left portion of each graph. The uncertainty of experimental data is presented as SEM. Figure adapted from Paper III. 
To understand how the model explains the change in vascular dynamics induced by anesthesia, we analyzed how the model explains the key characteristic observed in the experimental data: the prolonged post-peak undershoot due to the anesthetic effect in the case of an OG stimulation of GABAergic interneurons (Fig. 5.6A, black symbols). The model simulations reveal that the prolonged post-peak undershoot can be explained by two parts. Firstly, the anesthetic agent acts on the neuronal activity (Fig. 5.8A-B), changing the excitability of the two GABAergic neurons, inducing amplitude changes in the response, which are propagated through the intracellular signaling pathways (Fig. 5.8C-F). Secondly, the vasoconstrictive effect of NPY follows MichaelisMenten kinetics, converting the amplitude differences between awake and anesthesia at the neuronal level into duration changes at the level of the intracellular signaling pathways (Fig. 5.8C, E: compare awake with anesthesia). Finally, the two arms are summed together which forms the arteriolar diameter response (Fig. 5.8G). These amplitude and duration changes alter the arteriolar response from exhibiting a brief post-peak undershoot to a prolonged version, with minor changes in time to peak and peak amplitude (Fig. 5.8G: compare awake with anesthesia). For more details, see Paper III.
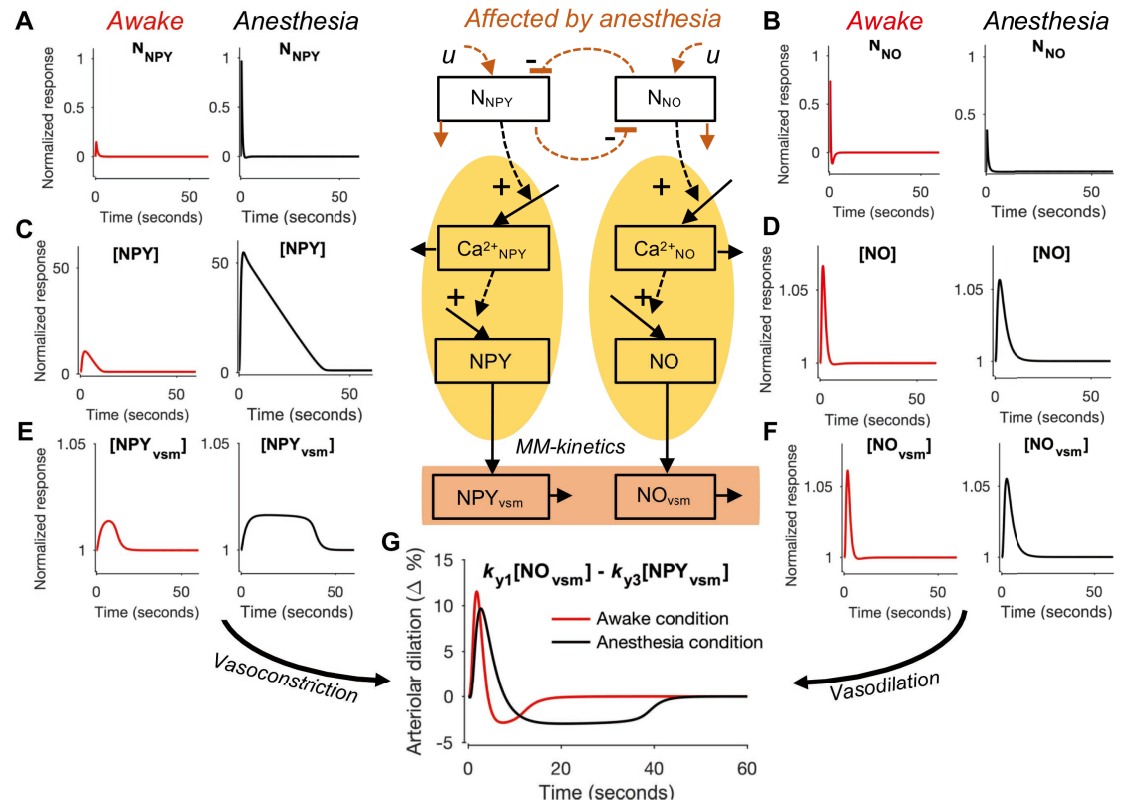

Figure 5.8: The model explanation of the anesthetic effect in the case of optogenetic stimulation of GABAergic interneurons. In the middle, a model schematic of the signaling pathways, and which reactions that are altered with anesthesia is depicted. The relevant states are shown: the neuronal dynamics of NPY $(\mathbf{A})$ and NO (B) interneurons, the propagation to the vasoactive effect of respective neuron $(\mathbf{C}, \mathbf{E}$ and $\mathbf{D}, \mathbf{F})$, and finally the vascular response (G). For each of these states, dynamics for awake (left graph) and anesthetized (right graph) model simulations are shown. Figure adapted from Paper III. 


\subsection{Paper IV: the final model can describe most relevant NVC measures}

In Paper IV, we sought to extend the framework presented in Paper III to not only describe arteriolar diameter responses, but to incorporate most of the available hemodynamic NVC measures. This idea was driven by the fact that no currently existing model which describe the NVC can simultaneously account for a wide variety of data types (Fig. 5.9B), including: 1) compartmentalized CBV and CBF, 2) oxygen saturation measures (hemoglobin, BOLDfMRI), 3) optogenetic stimulation of specific neuronal types, and finally, 4) pharmacological perturbations of intracellular signaling, including anesthetics. This new model is a combination of the model in Paper III, the hemodynamics model of Barrett et al. [43-45], and the BOLD signal derivation of Griffeth et al. [228] (Fig. 5.9A-B). This new model have a quantitatively good agreement to experimental data used for model training from multiple sources and species (Fig. 5.9A; Fig. 3, 5, 6, 7 in Paper IV), and can correctly predict independent data used for validation (Fig. 5 \& 7 in Paper IV). Furthermore, this model can be used to examine complex behaviors observed in experimental data, such as in Figure 5.10.

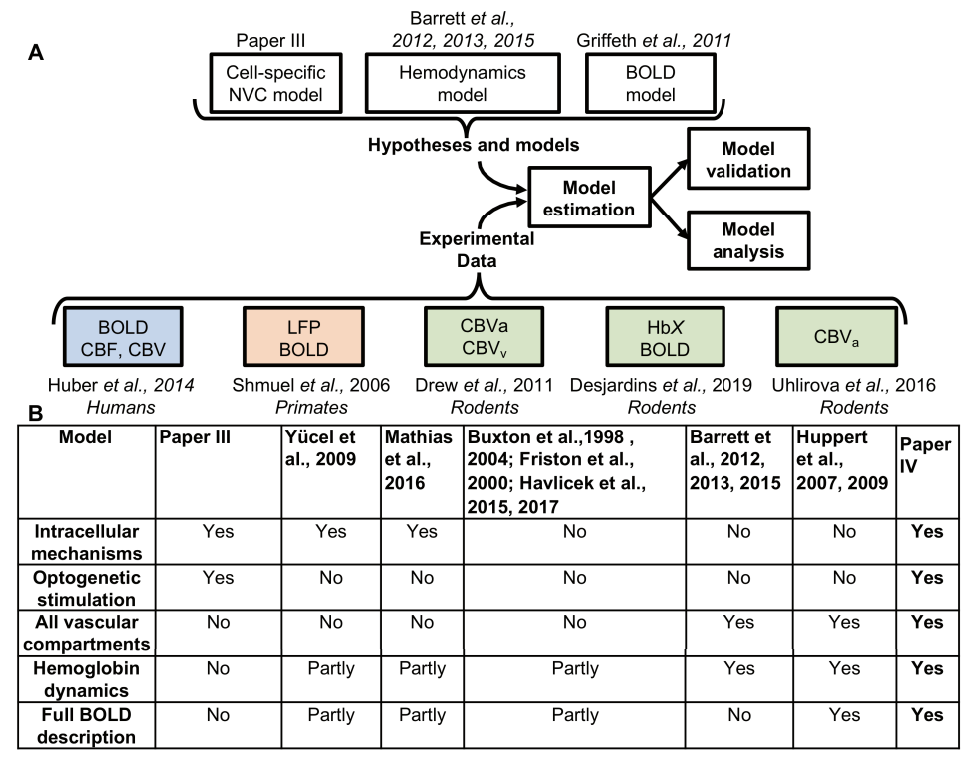

Figure 5.9: A: Overview of the study conducted in Paper IV. Experimental data from different species (colored boxes) and modalities are used to develop a new combined NVC model. B: Comparison of existing NVC models, with regards to different aspects. The notations used are: 'Yes' if the model features a satisfying description of the aspect; 'Partly' if the model features a description that is not fully satisfying but nevertheless exists, and 'No' if the model lacks a satisfying description of that aspect. Figure adapted from Paper IV. See Table 2.1 for full references. $\mathrm{HbX}$ hemoglobin measures; $\mathrm{CBV}_{i}$ compartment specific CBV. 
Here, the captured arteriolar responses to a sensory stimulation change from a one-peak (Fig. 5.10A) to a two-peak response (Fig. 5.10C), as the length of the stimulation increases. The model captures this transition between an uni-modal to a bi-modal response well (Fig. 5.10A-C, compare shaded areas and lines with symbols). Therefore, it is of interest to see how the model can replicate this behavior, by examining the last step before the arteriolar response: the summation of the vasoactive arms. In the model, the response to the shortest stimulation is produced by the fast release of NO from GABAergic interneurons, which produces the first peak in the arteriolar response (Fig. 5.10D-F, blue line) and which thereafter simply declines for the remainder of the stimulation. The other two signaling pathways exhibit slower kinetics, and are therefore negligible for the shortest stimulation. the dynamics of NO is also important for the two longer stimulations as the response declines quickly after the first peak. In the model, this is driven by the decline of NO, causing the decrease in arteriolar diameter observed after the first peak. For the two longer stimulations, a second peak is observed, which is driven by the slower $\mathrm{PGE}_{2}$ signaling arm from the pyramidal neuron (Fig. 5.10E-F, green line). Finally, the NPY signaling arm only comes into play in order to explain the post-peak undershoot observed for the longest stimulation (Fig. 5.10F, red line). In summary, the first peak in the arteriolar response is due to rapid $\mathrm{NO}$ arm, the second peak is due to the $\mathrm{PGE}_{2}$ arm, and finally, the post-peak undershoot (if observed) is due to the lingering effect of the NPY arm. This small example illustrates how these types of models can be used to investigate underlying mechanisms for observed complicated behaviors seen in various types of experimental data. For more details, see Paper IV. 


\subsection{5 s stimulation}

10 s stimulation A Arterioles B
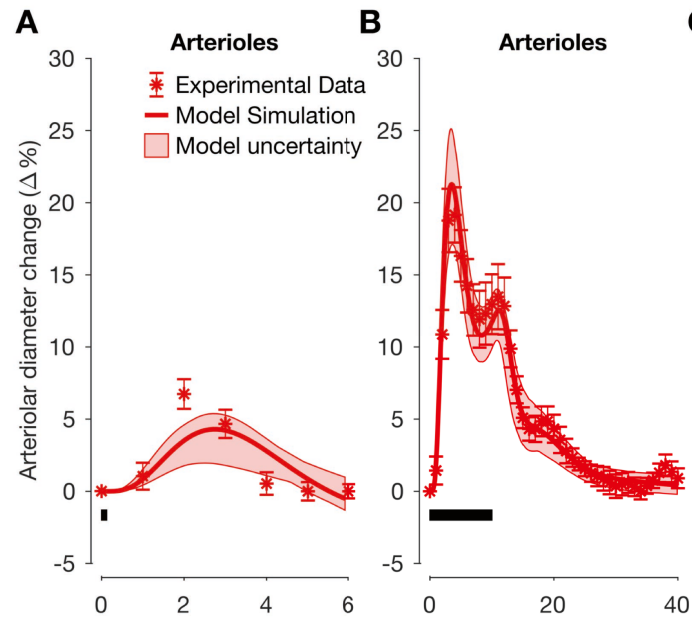

\section{0 s stimulation}
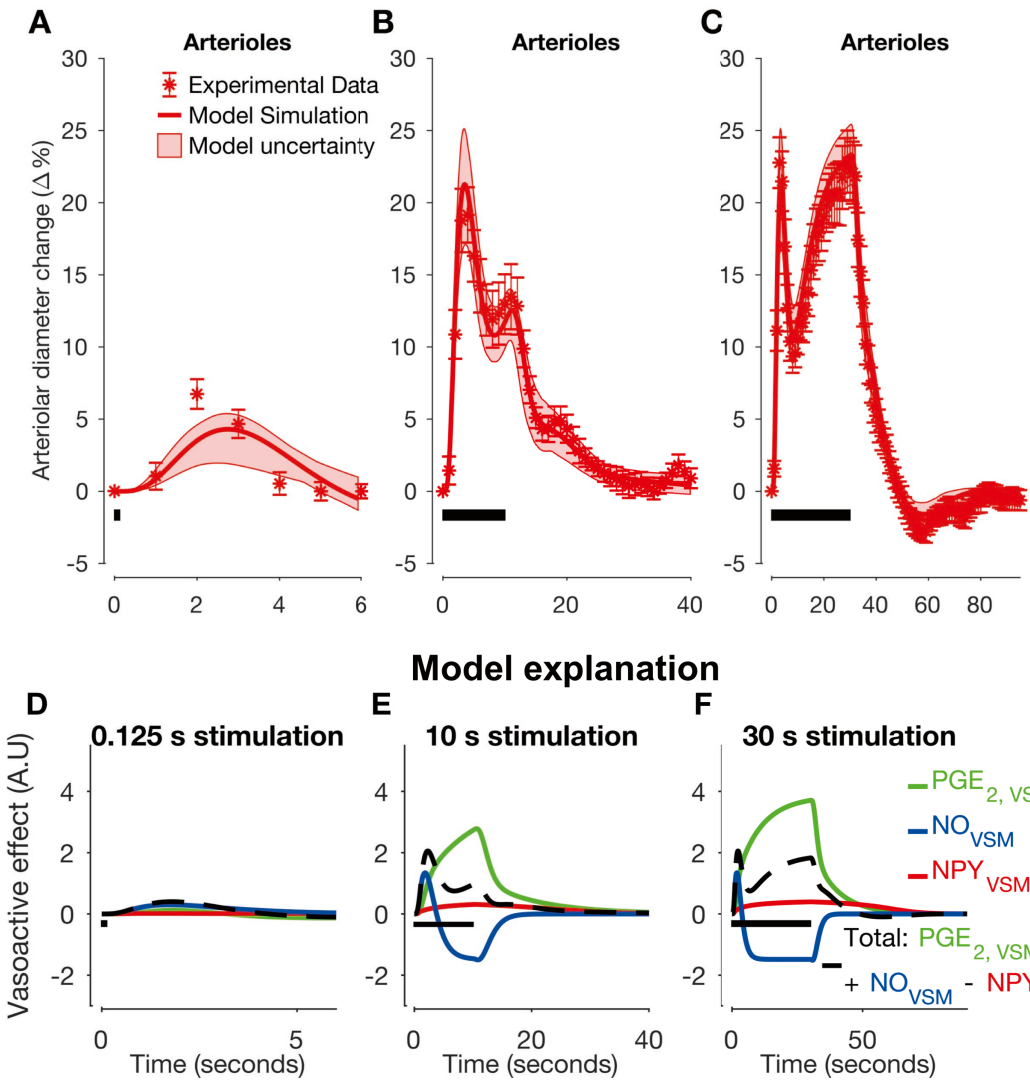

Model explanation

E
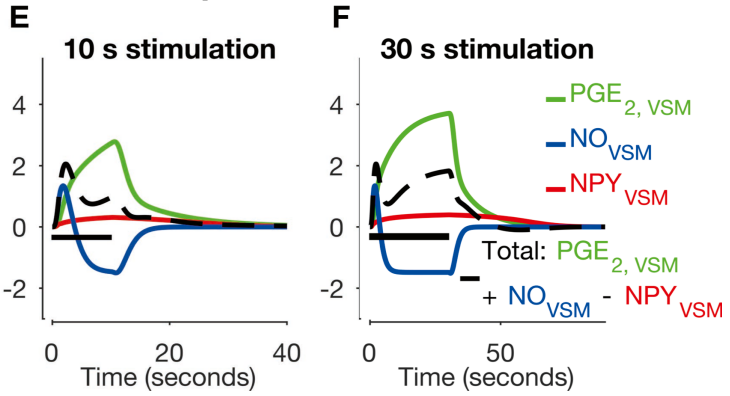

Figure 5.10: Model estimation and explanation of experimental data consisting of arteriolar (A-

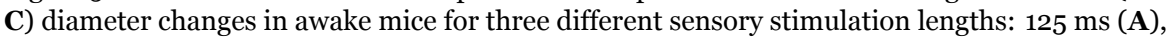
$10 \mathrm{~s}(\mathbf{B})$, and $30 \mathrm{~s}(\mathbf{C})$. For each graph: experimental data (mean \pm SEM) as red symbols; the best model simulation is seen as the red solid line, and the model uncertainty as the red shaded area. D-F: Model suggested explanation of the transition from a one-peak to a two-peak arteriolar response. Model simulations for the three stimuli lengths: $125 \mathrm{~ms}(\mathbf{D}), 10 \mathrm{~s}(\mathbf{E})$, and $30 \mathrm{~s}(\mathbf{F})$ are shown. For each graph: model simulations (colored lines), and length of the stimulation (black bar). The $\mathrm{x}$-axis depicts time, and the $\mathrm{y}$-axis depicts the vasoactive effect on the arteriolar compartment (A.U). Figure adapted from Paper IV. 


\section{6}

\section{Discussion and concluding remarks}

In this chapter, I will summarize the main findings from my papers, followed by a discussion of how these fit into the field of NVC research. I will also discuss some key limitations of my work, and provide some thoughts on the future outlook of my findings.

\subsection{Summary of main findings and conclusions}

\section{Paper I}

In Paper I, we show that neither of the metabolic feedback or vasoactive feedforward hypotheses could on their own both quantitatively and qualitatively describe the experimental data. A combination of key aspects from both hypotheses could describe data used for estimation, as well as predict independent data used for validation.

\section{Paper II}

A model implementation of the neuronal inhibition hypothesis as underlying negative BOLD responses could describe data used for training, and successfully predict independent data used for validation. This model constitutes one of the first mathematical models investigating the underpinnings of the negative BOLD response.

\section{Paper III}

In Paper III, we provide a first quantitative model of the cell-specific contributions to the NVC, including an explanation of the shift in dynamics that 
occur with the use of an anesthetic agent. The analysis suggests that one of the GABAergic interneuron signaling pathways, NPY signaling, must include a saturating expression that translates differences on the rapid neuronal level to be converted to duration changes on the vascular level.

\section{Paper IV}

In Paper IV, we extend the model structure presented in Paper III to describe a majority of the existing relevant NVC measures via the addition of a vascular model consisting of: blood volumes and flows, oxygen transport and saturation, hemoglobin dynamics, and finally, the addition of a BOLD-fMRI signal. The model can describe and predict experimental data from rodents, primates, and humans, and suggest explanations for complex behaviors observed in experimental data. This model constitutes the most complete mechanistic model of the NVC to date.

\subsection{The three phases of the BOLD response}

As described already in Chapter 2, there are three phases to the archetypal BOLD response: the initial dip, the main peak or overshoot, and the postpeak undershoot (Fig. 2.6A). Before the thesis, no mechanistic model existed that explained plausible biological mechanisms that cause these three phases. With the results of the four papers in this thesis, we can now provide an updated mechanistic understanding of how these three phases are generated.

\section{The initial dip}

It is debated whether the initial dip even exists, because if it exists it is comparable in size to measurement noise (see $[125,126]$ ). For this reason, only Paper I is concerned with the initial dip observed in BOLD-fMRI. In this paper, we find that the feed-forward signaling, caused by secretion of vasoactive substances, can in principle explain the initial dip. However, we reject that hypothesis, because it would imply that vasoconstrictive signaling happens directly within the first two seconds. Instead, we choose a model where the initial dip is caused by an initial rapid increase in oxygen metabolism.

\section{The main overshoot}

The increase in metabolism that causes the initial dip is in our models not causing the main overshoot component. This stands in contrast to the initially held belief of how the BOLD signal is generated. This initial belief was centered around a negative feedback: because the metabolism causes a drop in blood oxygenation, the blood flow increases thanks to a negative feedback that is a part of a control system (See Chapter 3 , 'The discovery of the NVC'). In 
Paper 1, we reject this negative feedback hypothesis. This rejection was based on the fact that all implementations of the negative feedback hypothesis that could explain the main overshoot also had an unrealistic prediction: they predicted that the metabolite that was involved in the feedback went down to zero for several seconds (Fig. 5.2B, D). For this reason, we concluded that the main overshoot was caused by a feed-forward system involving intracellular signaling which leads to the secretion of vasoactive substances. This conclusion was supported by the fact that additional model analysis revealed no further apparent unrealistic model predictions, and that the model could explain both estimation data and predict independent validation data not used for model training (Fig. 5.3).

In Papers III \& IV, we could return to these intracellular signaling pathways and unravel its more detailed composition by using pre-clinical data from mice. The most important component of those data is optogenetic perturbations, which allows us to more closely examine the role of different neuronal types. This analysis leads to a model, where the main response is caused by a combination of pyramidal neurons, which secrete $\mathrm{PGE}_{2}$, and interneurons which secrete NO and NPY. In Paper IV, we could refine this dual role further. For a short stimulation, the initial response regulates arterioles via the NO pathway. For longer stimuli, the NO pathway quickly subsides, and is overtaken by the $\mathrm{PGE}_{2}$ pathway. In certain conditions, this leads to a bimodal response, featuring two-peaks for the longer stimulations (Fig. 5.10).

\section{The post-peak undershoot}

In Paper I-II, the post-peak undershoot can be explained by the interplay between two vasoactive arms: one dilating and one constricting (see the minimized model in Paper 1, and the model in Paper II). These two arms exhibit highly similar dynamics, but the constricting arm has a prolonged time-scale, and is lower in magnitude. In total, the constricting arm briefly lingers on after the main response is over, causing the post-peak undershoot.

In Paper III \& IV, we unravel the nature of these two arms in much more detail. Using the optogenetic perturbations, we can find conditions where the post-peak undershoot is observed or not, and also find conditions that impact the duration of the post-peak undershoot. Using these data, we are able to elucidate that the post-peak undershoot is caused by the NPY pathway. Furthermore, we can identify that this NPY pathway needs to have a saturation, since there are situations where the post-peak undershoot is prolonged but without an increased post-peak undershoot amplitude (Fig. 5.7). In Paper IV, this mechanistic unraveling is further refined by looking at data that specifically looks at the arteriolar diameter. For long stimuli, the arteriolar diameter has both a bi-modal main response, and a post-peak undershoot. 
Our analysis based on this data again confirms that the post-peak undershoot is caused by the NPY pathway, which is the slowest of the three arms. In other words, compared to the situation in Paper I, we now know that the hypothesis of two vasoactive arms with different time scales is supported by detailed experimental data, and that the negative arm is made out of the slow NPY pathway.

Apart from our conclusions, there are also other explanations suggested in the literature that are worth mentioning. More specifically, the post-peak undershoot of the BOLD response can be caused by a slow return of venous blood volume for very long stimulations. In such cases, the post-peak undershoot is caused by a big blood volume in the venous compartment, which is filled with large amounts of deoxyhemoglobin. Because of this slow deoxyhemoglobin dynamic, the post-peak undershoot can appear in the fMRI-BOLD signal without it being present for CBF. For more details, see [229, 230]. Note however, the data studied here clearly shows that you only have a substantial venous volume for long stimuli (Paper IV, Fig. $3 \&$ 7).

\subsection{The three vascular compartments}

There are three vascular compartments involved in the NVC: the arterioles, the capillaries, and the venules. These three compartments have different dynamics and regulations, and are all included in our final model (Paper IV). Let us therefore briefly consider them one by one.

\section{Arterioles}

Arteriole dynamics have been well characterized in rodent data used in Paper III and IV. These data include different optogenetic stimuli (pyramidal neurons, GABAergic interneurons), sensory stimuli, the addition of pharmacological inhibitors, and measurements for both awake and sedated conditions. In Paper III, we are able to explain all of these data, including independent validation data, and we therein provide a mechanistic explanation for how the different observed dynamics can be caused. In particular, we provide a first explanation for the difference between awake and sedated conditions. This is important since a large majority of data is available only for anesthesia conditions, and because anesthetics impact the mechanisms involved in the NVC. A part of this explanation concerns the different dynamics observed for optogenetic perturbations of GABAergic interneurons in awake and anesthesia conditions: a prolonged post-peak undershoot is observed for the sedated condition (Fig. 5.6A). Our analysis shows that this prolonged post-peak undershoot spanning more than 20 seconds is caused by a difference in amplitude during the short optogenetic stimulation through a sequence of three steps: 1) the difference in amplitude is propagated through the NPY pathway 
and leads to a difference in the duration of the response; 2) the duration is preserved while the corresponding differences in amplitude is removed through a saturated step somewhere in the NPY pathway; 3) the entire response is caused by the sum of the NPY pathway and the NO pathway (Fig. 5.8).

In Paper IV, we further refine our understanding for the regulation of the arterioles. For short responses, there is a single peak which for increasing longer stimuli transforms into a bi-modal response of two peaks, and a postpeak undershoot. The explanation of these two phases is explained in Chapter 6.2, 'The main overshoot' and 'The post-peak undershoot', respectively. In Paper IV, we also considered arterial CBV data for humans. Unlike for arteriolar responses in mice, no bi-modal response is observed in humans, which instead exhibits a simple single peak response.

\section{Capillaries}

Not included in the thesis, we have a preliminary model of direct capillary regulation. However, no applicable in vivo data is available for this regulation, and therefore we choose not to include it in Paper IV. Instead, the capillary regulation in the model in Paper IV is exclusively caused by the vascular model propagating changes in blood pressure and flow from the arterioles to the capillaries. No plots are included in the paper, but the simulations display dynamics lying between the arteriolar and venular responses.

\section{Venules}

The venules are only studied in Paper IV. In Figure 3D-F in Paper IV, we see responses for short, intermediate, and long stimulations. For the shortest stimulation (125 ms), a hardly observable single peak response can be seen. For the longer stimulations, more dynamics can be observed. For the longest stimulation (30 s), a clearly defined single peak response is observed, which is explained well by the model. For the intermediate stimulation (10 s), the situation is more unclear: there may be a more complicated behavior seen in the data, but since the signal is almost below the detection limit (see Discussion in Paper IV), we choose to primarily present model simulations that anyway show a simple single peak response. Just as for the capillaries, the venules have no direct control, but their dynamics are simply caused by the vascular model propagating control from the arterioles. In Paper IV, we also study human data for venous responses (see Fig. 7 in Paper IV). For the positive BOLD, the venous response is highly similar to the arterial response with a single peak response. For the negative BOLD response, the behavior may be more complex, but the uncertainty of the data is high, and therefore model simulations show a simple response. Finally, our model stands in stark contrast to the widely studied Balloon model, which only describes the dynamics 
of a venous compartment, even though the venous compartment has no direct control.

\subsection{Negative BOLD}

In Paper II, we examine the negative BOLD response for the first time. The model presented is a simple extension of the feed-forward model of Paper I. The primary extension consists of the addition of GABA, which inhibits instead of excites, the neural cells. This extension allows us to explain human data for both the positive and the negative BOLD response. The model structure in these two responses is the same, but we assume that some of the parameters for the intracellular signaling are allowed to change. This is an important weakness of this work. Nevertheless, the model is able to explain estimation data and independent validation data, in a way that is supported by statistical tests.

In Paper IV, we again return to the negative BOLD response by examining data from primates and humans. In data collected in primates (see [221]), it is seen that the negative BOLD response is accompanied by a corresponding negative deflection in the LFP. This supports our initial model, which also shows such a correlation between electrical activity and the BOLD response for the positive and negative BOLD response, respectively. Again our model is able to explain these data, and this time using the more advanced model structure that can explain all other data in Paper IV. Importantly, in contrast to the model in Paper II, no parameters are changed in the intracellular signaling between the positive and negative responses. Our initial simple explanation is further supported by the human data in Figure 7 in Paper IV. Here, the negative BOLD is captured with corresponding measures of CBV. While the CBV data for the two compartments, arteries and veins, are noise-polluted, the total CBV time series is well defined. It shows a similar decrease from baseline as the BOLD response. In summary, in all situations encountered in this thesis, the negative BOLD response is caused by a decrease already at the electrophysiological level, which is propagated to a similar drop in CBF and CBV. Note that the drop in blood flow is a prediction by the model, which is supported by independent validation data (Paper IV, Fig. 7E).

\subsection{Limitations}

As with all models, there exist a number of assumptions and limitations, which limit the conclusions that can be drawn. These limitations are now discussed on the general level for all of the presented models in this thesis. 


\section{Neuronal activity}

First, our description of neuronal activity is quite simple throughout the different papers. For Paper I \& II, we express the unspecified release of glutamate and GABA, and in Paper III \& IV we employ a simple phenomenological relationship between GABAergic interneurons and pyramidal neurons. A more correct interpretation of neuronal activity would be to integrate our models with existing physical models of membrane potential fluctuations (see [231] and references therein). Furthermore, such a combination would open the door to including the structural and functional morphologies of specific subsets of interneurons (see [86]). This stands in contrast to the current lumped approach used in Paper III \& IV, where we simply model an average NPY and NO interneuron.

\section{Metabolism}

Another highly simplified aspect of the presented models concerns cerebral metabolism. While metabolism of both oxygen (Paper I-II, IV) and glucose (Paper I-II) occurs in some of the models, this aspect could be expanded in two ways: 1) to include the commonly measured cerebral metabolites, often captured using magnetic resonance spectroscopy; 2) to include direct in vivo measures of tissue oxygen pressure. This would lead to a model capable of a detailed description of the ongoing metabolic processes during increased neuronal activity, and could further be extended to describe the energy consumption as a function of the opening and closing of ion channels during action potential firing.

\section{The use of arbitrary units}

Throughout the different models, the states are often specified in arbitrary units, meaning that the states represent biological values such as concentrations or amounts, but these are scaled by unknown constants. This limitation is often due to corresponding limitations of experimental data, as most of these intracellular molecules are hard to measure experimentally both in vitro and in vivo, and if measurable, this measurement often only captures relative changes.

\section{Re-fitting of parameters between datasets}

An important strength of this modeling is the use of independent validation data. In this usage, the parameters are trained on specific estimation data which determines the values of all the parameters. Then, without changing any parameter values, predictions are compared with new data, which are not a part of the estimation data. In contrast, there are important cases when 
the parameters have been allowed to change between experiments, and these changes should be clearly specified.

In Paper I, two sets of estimation and validation data are used. In the first dataset (intensity), the model is trained on a single response and validated on other data where the contrast of the visual cue is lower. In the second dataset (frequency), the model is trained on a similar single response, but this time the validation data consists of a set of visual cues, with a $1 \mathrm{~s}$ or $4 \mathrm{~s}$ delay inbetween the two cues. The parameters are held the same within these two datasets, but are allowed to be different between the two datasets.

In Paper II, there are also two different studies included. The first dataset (as published in [232]; Fig. 4 in Paper II) was used to develop and refine the model. The second dataset (as published in [233, 234]; Fig. 5 in Paper II) comes from another study, which means that the parameters were re-fitted when considering these data. Furthermore, the second dataset was subdivided into two parts: one part was used to re-fit the model to the new experimental setting, and the second part was used to validate the predictive ability of the model.

In Paper III, all data came from a single study ([88]). For this reason, we kept the parameters essentially the same for all data. The only difference in parameters allowed was for the electrophysiological part, which was allowed to be different between awake and anesthesia conditions. This difference is important because awake and anesthesia conditions do imply important differences in brain dynamics, which the paper sought to explore. However, the intracellular signaling was assumed to be the same for the two conditions. As usual, the complete set of data was subdivided into two parts: one part for estimation and one part for validation, and the parameters were kept the same when comparing simulations with the independent validation data.

In Paper IV, experimental data from many different sources were included. As usual, the general principle was that the parameters were allowed to change between experimental settings. This is reasonable, since different experimental settings do give rise to different observed dynamics, such as the presence or absence of a post-peak undershoot. Nevertheless, to utilize the fact that the same model structure could agree with this wide variety of data, we sought to make the model behave in a similar way when moving from one dataset to another.

Finally, we assume that most of the parameters are not known from before and thus should be estimated from data. This concerns all of the models in Papers I-III. For Paper IV, we re-used two models, one for the three vascular compartments, and one for the calculation of the BOLD signal. For both of 
these re-used models, the parameters were retained from previous publications.

\subsection{Future outlook}

\section{Connections to the electrophysiological and neuronal network models}

Since the introduction of the Hodgkin-Huxley models, neuroscience has had a long tradition of applying mathematical models in order to understand and generate new insights regarding the inner workings of the brain. Since the original papers, these models have become increasingly realistic and complex. On the intracellular level, extensive experimental studies have unraveled the detailed mechanisms of ion channels. On the cell-cell communication level, models include gap-junction couplings, which describe communications between cells. Finally, on the whole-brain level, models describe physiological neural networks which perform central brain functions, such as object recognition and control of muscular actions [235-237]. One interesting such example is the object recognition models by Grossberg et al., which describe the interplay between different brain regions in the complex task of recognizing objects when an eye is looking at different parts of an object (see [237] and references therein). Another fascinating example is the 'BabyX' project by Mark Sager et al. [238, 239]. This model describes many of the functions of a small child, such as interpretation and recognition of sounds, different emotional responses to different stimuli, and facial mimicking of the persons that the child is observing. All of these functions are simulated by the 'BabyX' model, in a way that involves the corresponding brain regions. However, none of these models include a realistic description of NVC. It is therefore an interesting challenge to connect our already existing NVC model with some of the above-mentioned models for electrophysiology and brain function.

\section{Surpassing the capabilities of all other existing models for the NVC}

There are some existing models for the NVC which are important to mention in this context, because these models are used for certain applications that we have not considered in this thesis. One of the most important such applications is displayed by the simple model by Davis et al. [127]. This model is important because it can combine data for the BOLD signal with CBF data in order to calculate an estimation of $\mathrm{CMRO}_{2}$. These three entities - BOLD, $\mathrm{CBF}$, and $\mathrm{CMRO}_{2}-$ are also included in our models, but we have not explored whether our models can be used for a similar purpose. In general, the combination of a variety of different data sources that describe different aspects 
of NVC into the same model is one of the most important future potentials of our model. Using such data integration, it is likely that future versions of our model will be able to draw conclusions about mechanisms and the biomedical status of the brain, which have previously not been possible to obtain. This can open the door to many important applications within the clinic, basic research, and for the development of new treatments.

\section{Clinical usage}

Today, fMRI data is analyzed in a highly simplistic manner, which does not incorporate any mechanistic understanding of the biology involved. The current analysis is typically done by using the GLM approach, which looks at the correlation between observed fMRI-BOLD signal and the HRF (Fig. 2.7). This method equates brain activity with a high correlation: if the correlation is above a certain threshold, it is assumed that brain activity is present. This approach has a number of shortcomings. First of all, it does not make use of any biological understanding, even though much biological knowledge regarding the NVC is available. Second, because of the first shortcoming, this method can not incorporate other types of data into the same model. For this reason, the traditional GLM based approach can probably never go beyond a phenomenological assessment of brain activity to a more nuanced understanding of subtle differences between different brain areas or changes in the NVC. The model presented herein can, once sufficiently validated and analyzed, fundamentally improve this situation. In the future, we predict that it will be possible to use a more versatile MRI paradigm which is capable of collecting a wider array of data types and which can produce a battery of currently not available biomarkers. An important challenge for future research is to make this potential reality and to explore the power of such new biomarkers, both for direct clinical applications in diagnosis, prognosis, and patient stratification, and finally also for the identification of new treatments.

\section{New treatments}

We believe that our new model-based data analysis can be used to guide research and development of a wide variety of treatments. For instance, an improved mechanistic understanding of the neurological underpinnings of central neurological diseases can help guide pharmaceutical research. For this to become a reality, some important developments remain: the model e.g. needs to be adopted to different diseases, and a pharmacokinetic component describing the dynamics of potential drugs needs to be developed. Such model-based drug development is already a reality in most drug development companies, even though the most widely used application today is for pharmacokinetic models. Here, our research could open the door for more physiologically based modeling of the impact of the drug i.e., pharmaco- 
dynamic modeling. Finally, regarding pharmacological research, our model can in principle also become useful in the final approval stage, since the food and drug administration in the United States has now published guidelines for how and when models can be used in approval settings, e.g. to replace animal experiments or to reduce the size of clinical trials [240-242]. Apart from pharmacological treatments, our model could also be used to study other types of treatments commonly used to treat neurological diseases. Such alternative treatments could for instance include cognitive behavior therapy, psycho-dynamic treatments, and meditation. It is an interesting challenge for future research to use Systems Biology to provide a mechanistic underpinning for such alternative treatments. 



\section{Bibliography}

[1] Eric R Kandel. Principles of neural science. English. 2013. isbn: 97800713901180071390111.

[2] A. L. Hodgkin and A. F. Huxley. Action potentials recorded from inside a nerve fibre. 1939. doi: 10.1038/144710a0.

[3] G Cimino. "Reticular theory versus neuron theory in the work of Camillo Golgi." eng. In: Physis; rivista internazionale di storia della scienza 36.2 (1999), pp. 431-472. issn: 0031-9414 (Print).

[4] Frank W. Stahnisch. "Joseph von Gerlach (1820-1896)". In: Journal of Neurology 262.5 (May 2015), pp. 1397-1399. issn: 14321459. doi: 10. 1007/s00415-015-7735-2. url: https ://link. springer.com/ article/10.1007/s00415-015-7735-2.

[5] Camillo Golgi. Sulla fina anatomia degli organi centrali del sistema nervoso. S. Calderini, 1885.

[6] Gunnar Grant. How the 1906 Nobel Prize in Physiology or Medicine was shared between Golgi and Cajal. 2007. doi: 10 . 1016 / j . brainresrev . 2006.11.004. url: https://pubmed.ncbi.nlm.nih . gov/17306375/.

[7] Mitch Glickstein. Golgi and Cajal: The neuron doctrine and the 10oth anniversary of the 1906 Nobel Prize. Mar. 2006. doi: 10.1016/ j. cub. 2006.02.053.

[8] Quasar Jarosz. Neuron Hand-tuned.svg - Wikimedia Commons. url: https : / / commons . wikimedia . org / wiki / File : Neuron_ Handtuned.svg (visited on 06/08/2020).

[9] Wei-Chung Allen Lee, Hayden Huang, Guoping Feng, Joshua R Sanes, Emery N Brown, Peter T So, and Elly Nedivi. "Dynamic Remodeling of Dendritic Arbors in GABAergic Interneurons of Adult Visual Cortex". In: PLoS Biology 4.2 (Dec. 2005). Ed. by Charles F. 
Stevens, e29. issn: 1545-7885. doi: 10.1371/journal .pbio. 0040029. url: https ://dx.plos .org/10.1371/journal . pbio. 0040029 .

[10] Bente Pakkenberg, Dorte Pelvig, Lisbeth Marner, Mads J. Bundgaard, Hans Jørgen G. Gundersen, Jens R. Nyengaard, and Lisbeth Regeur. "Aging and the human neocortex". In: Experimental Gerontology. Vol. 38. 1-2. Pergamon, Jan. 2003, pp. 95-99. doi: 10.1016/S0531-5565(02)00151-1.

[11] United States National Library of Medicine. The Visible Human Project. 1994. url: ftp : / / lhcftp . nlm . nih . gov/Open-Access Datasets / Visible - Human / Male - Images / PNG_format / head / a vm1054.png.

[12] John Batty Tuke. The insanity of over-exertion of the brain. France, Europe: Oliver and Boyd (Edinburgh), 1894, p. 66. url: https : // login.e.bibl . liu. se/login?url=https ://search . ebscohost . com/login. aspx ?direct $=$ true \&db=edsbas \&AN=edsbas . 7A3D3D77\& site=eds-live\&scope=site.

[13] V. B. MOUNTCASTLE. "Modality and topographic properties of single neurons of cat's somatic sensory cortex.” In: Journal of neurophysiology 20.4 (July 1957), pp. 408-434. issn: 00223077. doi: 10 . 1152/jn.1957.20.4.408.

[14] Korbinian Brodmann. Vergleichende Lokalisationslehre der Grosshirnrinde in ihren Prinzipien dargestellt auf Grund des Zellenbaues. German. Leipzig: Barth, 1909.

[15] Mark F. Bear, Barry W. Connors, and Michael A. Paradiso. Neuroscience : exploring the brain. Lippincott Williams \& Wilkins, 2002. isbn: 0781731321.

[16] University of Michigan. The Human Brain. url: http://umich.edu/ $\$ \% 5$ Csim\$cogneuro/jpg/Brodmann.html (visited on 06/09/2020).

[17] Marcus E. Raichle. "The restless brain: how intrinsic activity organizes brain function". In: Philosophical Transactions of the Royal Society B: Biological Sciences 370.1668 (May 2015), p. 20140172. issn: 0962-8436. doi: 10.1098/rstb. 2014.0172.

[18] DD Clarke and L Sokoloff. Circulation and energy metabolism of the brain. In: Basic Neurochemistry. Ed. by Albers RW Siegel GJ, Agranoff BW. 6th. Philadelphia: Lippincott-Raven, 1994. isbn: ISBN-10: 0-397-51820-X.

[19] Angelo. Mosso. Ueber den Kreislauf des Blutes im Menschlichen Gehirn : Untersuchungen. German. Leipzig: Veit, 1881. 
[20] C. S. Roy and C. S. Sherrington. "On the Regulation of the Blood $\square$ supply of the Brain". In: The Journal of Physiology 11.1-2 (1890), pp. i-xiv, 1-158. issn: 14697793. doi: 10 .1113/ jphysiol. 1890 . sp000321.

[21] Costantino Iadecola. "Neurovascular regulation in the normal brain and in Alzheimer's disease". In: Nature Reviews Neuroscience 55.5 (2004), pp. 347-36o. issn: 1471003X. doi: 10.1038/nrn1387.

[22] Costantino Iadecola. "The Neurovascular Unit Coming of Age: A Journey through Neurovascular Coupling in Health and Disease." In: Neuron 96.1 (Sept. 2017), pp. 17-42. issn: 1097-4199 (Electronic); 0896-6273 (Linking). doi: 10.1016/j . neuron. 2017.07.030.

[23] Bruno Cauli and Edith Hamel. "Revisiting the Role of Neurons in Neurovascular Coupling”. In: Frontiers in Neuroenergetics 2 (2010), p. 9. doi: 10.3389/fnene.2010.00009. url: http://www .ncbi.nlm . nih.gov/pmc/articles/PMC2899521/.

[24] David Attwell, Alastair M Buchan, Serge Charpak, Martin Lauritzen, Brian A Macvicar, and Eric A Newman. "Glial and neuronal control of brain blood flow.” In: Nature 468.7321 (2010), pp. 232-243. issn: o028-0836. doi: 10.1038/nature09613.

[25] Elizabeth M C Hillman. "Coupling mechanism and significance of the BOLD signal: a status report.” In: Annu Rev Neurosci 37 (2014), pp. 161-181. issn: 1545-4126 (Electronic); 0147-006X (Linking). doi: 10.1146/annurev-neuro-071013-014111.

[26] Amy R. Nippert, Kyle R. Biesecker, and Eric A. Newman. Mechanisms Mediating Functional Hyperemia in the Brain. Feb. 2018. doi: 10 . 1177/1073858417703033.

[27] Nikos K. Logothetis and Brian A. Wandell. "Interpreting the BOLD Signal”. In: Annual Review of Physiology 66.1 (Mar. 2004), pp. 735769. issn: 0066-4278. doi: 10.1146/annurev . physiol.66.082602. 092845.

[28] S Ogawa, T M Lee, A R Kay, and D W Tank. "Brain magnetic resonance imaging with contrast dependent on blood oxygenation." In: $P$ Natl Acad Sci Usa 87.24 (1990), pp. 9868-9872. issn: 0027-8424.

[29] Peter A. Bandettini, Eric C. Wong, R. Scott Hinks, Ronald S. Tikofsky, and James S. Hyde. "Time course EPI of human brain function during task activation”. In: Magnetic Resonance in Medicine 25.2 (1992), pp. 390-397. issn: 15222594. doi: 10.1002/mrm. 1910250220.

[30] Arno Villringer and Britton Chance. "Non-invasive optical spectroscopy and imaging of human brain function". In: Trends in Neurosciences 20.10 (Oct. 1997), pp. 435-442. issn: 01662236. doi: 10. 1016/S0166-2236 (97) 01132-6. 
[31] Joana B. Balardin, Guilherme A. Zimeo Morais, Rogério A. Furucho, Lucas Trambaiolli, Patricia Vanzella, Claudinei Biazoli, and João R. Sato. "Imaging brain function with functional near-infrared spectroscopy in unconstrained environments". In: Frontiers in Human Neuroscience 11 (May 2017). issn: 16625161. doi: 10 . 3389/fnhum . 2017.00258.

[32] Y Hoshi. "Chapter 7 - Hemodynamic signals in fNIRS”. In: New Horizons in Neurovascular Coupling: A Bridge Between Brain Circulation and Neural Plasticity. Ed. by Kazuto Masamoto, Hajime Hirase, and Katsuya B T - Progress in Brain Research Yamada. Vol. 225. Elsevier, 2016, pp. 153-179. isbn: o079-6123. doi: https : //doi .org/ 10.1016/bs . pbr. 2016.03.004. url: http: //www . sciencedirect . com/science/article/pii/S007961231600042X.

[33] Peter A. Bandettini. Twenty years of functional MRI: The science and the stories. Aug. 2012. doi: 10.1016/j . neuroimage . 2012.04 . 026. url: https://pubmed.ncbi.nlm.nih.gov/22542637/.

[34] K J Friston, A P Holmes, K J Worsley, J.-P. Poline, C D Frith, and R S J Frackowiak. "Statistical parametric maps in functional imaging: A general linear approach". In: Human Brain Mapping 2.4 (Jan. 1994), pp. 189-210. issn: 1065-9471. doi: 10 . 1002 / hbm . 460020402. url: https://doi.org/10.1002/hbm. 460020402.

[35] K J Friston. Statistical parametric mapping : the analysis of functional brain images. English. Amsterdam; Boston: Elsevier / Academic Press, 2007. isbn: 97801237256080123725607.

[36] Hiroaki Kitano. Systems biology: A brief overview. Mar. 2002. doi: 10.1126/science. 1069492.

[37] Hiroaki Kitano. Computational systems biology. Nov. 2002. doi: 10 . 1038/nature01254.

[38] Peter K Sorger. “A reductionist's systems biology: opinion.” In: Current opinion in cell biology 17.1 (Feb. 2005), pp. 9-11. issn: 09550674. doi: 10.1016/j . ceb. 2004 . 12 . 012. url: http: / / www . ncbi . nlm.nih.gov/pubmed/15661513.

[39] R Johansson. Model-Based Hypothesis Testing in Biomedicine: How Systems Biology Can Drive the Growth of Scientific Knowledge. Linköping Studies in Science and Technology. Dissertations. Linköping University Electronic Press, 2017. isbn: 9789176854570. url: https://books . google. se/books?id=0qw4DwAAQBAJ.

[40] Richard B Buxton, Kâmil Uludağ, David J Dubowitz, and Thomas T Liu. "Modeling the hemodynamic response to brain activation". In: Neuroimage 23 (2004), S220-S233. issn: 1053-8119. doi: 10.1016/ j.neuroimage.2004.07.013. 
[41] K J Friston, A Mechelli, R Turner, and C J Price. "Nonlinear Responses in fMRI: The Balloon Model, Volterra Kernels, and Other Hemodynamics". In: Neuroimage 12.4 (2000), p. 466477. issn: 10538119. doi: 10.1006/nimg. 2000.0630.

[42] Martin Havlicek, Alard Roebroeck, Karl Friston, Anna Gardumi, Dimo Ivanov, and Kamil Uludag. "Physiologically informed dynamic causal modeling of fMRI data." In: Neuroimage 122 (2015), pp. 355372. issn: 1053-8119. doi: 10.1016/j . neuroimage . 2015.07.078.

[43] Matthew J.P. Barrett, Merryn H. Tawhai, and Vinod Suresh. "Arteries dominate volume changes during brief functional hyperemia: Evidence from mathematical modelling”. In: NeuroImage 62.1 (Aug. 2012), pp. 482-492. issn: 10538119. doi: $10.1016 / \mathrm{j}$. neuroimage . 2012 . 05 . 005. url: http : / / www . ncbi . nlm . nih . gov / pubmed / 22587899\%20https://linkinghub. elsevier.com/retrieve/pii/ S1053811912004934.

[44] Matthew J.P. Barrett and Vinod Suresh. "Extra Permeability is Required to Model Dynamic Oxygen Measurements: Evidence for Functional Recruitment?” In: Journal of Cerebral Blood Flow \& Metabolism 33.9 (Sept. 2013), pp. 1402-1411. issn: 0271-678X. doi: 10.1038/jcbfm. 2013.74.

[45] Matthew J.P. Barrett and Vinod Suresh. "Improving estimates of the cerebral metabolic rate of oxygen from optical imaging data". In: NeuroImage 106 (Feb. 2015), pp. 101-110. issn: 10959572. doi: 10 . 1016/j.neuroimage.2014.11.041. url: http://www.ncbi.nlm.nih . gov/pubmed/25463454.

[46] Theodore J Huppert, Monica S Allen, Heval Benav, Phill B Jones, and David A Boas. "A multicompartment vascular model for inferring baseline and functional changes in cerebral oxygen metabolism and arterial dilation." In: Journal of Cerebral Blood Flow \& Metabolism 27.6 (June 2007), pp. 1262-1279. issn: 0271-6780. doi: 10.1038/sj . jcbfm. 9600435 .

[47] Theodore J Huppert, Phill B Jones, Anna Devor, Andrew K Dunn, Ivan C Teng, Anders M Dale, and David A Boas. "Sensitivity of neuralhemodynamic coupling to alterations in cerebral blood flow during hypercapnia." In: J Biomed Opt 14.4 (2009), p. 44038. issn: 15602281. doi: $10.1117 / 1.3210779$.

[48] Theodore J. Huppert, Monica S. Allen, Solomon G. Diamond, and David A. Boas. "Estimating cerebral oxygen metabolism from fMRI with a dynamic multicompartment Windkessel model". In: Human Brain Mapping 30.5 (May 2009), pp. 1548-1567. issn: 10659471. doi: 10 . 1002/hbm . 20628. url: http : / / doi . wiley . com/10 . 1002/ hbm. 20628. 
[49] Meryem A Yucel, Anna Devor, Ata Akin, and David A Boas. "The Possible Role of $\mathrm{CO}(2)$ in Producing A Post-Stimulus CBF and BOLD Undershoot." In: Front Neuroenergetics 1 (2009), p. 7. issn: 1662-6427. doi: 10.3389/neuro.14.007.2009.

[50] E J Mathias, M J Plank, and T David. "A model of neurovascular coupling and the BOLD response: PART I." In: Comput Methods Biomech Biomed Engin (Nov. 2016), pp. 1-11. issn: 1476-8259 (Electronic); 1025-5842 (Linking). doi: 10 . 1080/10255842 . 2016 . 1255732.

[51] Elshin J Mathias, Allanah Kenny, Michael J Plank, and Tim David. "Integrated models of neurovascular coupling and BOLD signals: Responses for varying neural activations." In: Neuroimage 174 (July 2018), pp. 69-86. issn: 1095-9572 (Electronic); 1053-8119 (Linking). doi: $10.1016 / \mathrm{j}$.neuroimage .2018 .03 .010 .

[52] Richard B. Buxton, Eric C. Wong, and Lawrence R. Frank. "Dynamics of blood flow and oxygenation changes during brain activation: the balloon model." In: Magn Reson Med 39.6 (June 1998), pp. 855-864. issn: 0740-3194 (Print); 0740-3194 (Linking). doi: 10 . 1002 /mrm . 1910390602.

[53] Patrick J. Drew. Vascular and neural basis of the BOLD signal. Oct. 2019. doi: 10.1016/j. conb. 2019.06.004.

[54] Melanie Boly, Olivia Gosseries, Marcello Massimini, and Mario Rosanova. "Chapter 2 - Functional Neuroimaging Techniques". In: ed. by Steven Laureys, Olivia Gosseries, and Giulio B T - The Neurology of Conciousness (Second Edition) Tononi. San Diego: Academic Press, 2016, pp. 31-47. isbn: 978-0-12-800948-2. doi: https : / / doi . org / 10 . 1016 / B978-0 - 12-800948-2 .00002-9. url: http : / / www . sciencedirect . com / science / article / pii / B9780128009482000029.

[55] Michael I Posner and Marcus E Raichle. Images of mind. New York, NY, US: Scientific American Library/Scientific American Books, 1994, pp. ix, 257-ix, 257. isbn: 0-7167-5045-7 (Hardcover).

[56] Seymour S. Kety. Circulation and metabolism of the human brain in health and disease. Feb. 1950. doi: 10.1016/0002-9343(50)903639.

[57] Christoph Leithner and Georg Royl. The oxygen paradox of neurovascular coupling. Jan. 2014. doi: 10.1038/j jbfm . 2013.181. url: http://journals. sagepub.com/doi/10.1038/jcbfm.2013.181. 
[58] Dov Malonek and Amiram Grinvald. "Interactions between electrical activity and cortical microcirculation revealed by imaging spectroscopy: Implications for functional brain mapping". In: Science 272.5261 (Apr. 1996), pp. 551-554. issn: 00368075. doi: 10.1126/ science.272.5261.551.

[59] Nikolas Offenhauser, Kirsten Thomsen, Kirsten Caesar, and Martin Lauritzen. "Activity-induced tissue oxygenation changes in rat cerebellar cortex: Interplay of postsynaptic activation and blood flow". In: Journal of Physiology 565.1 (May 2005), pp. 279-294. issn: 00223751. doi: 10.1113/jphysiol.2005.082776.

[6o] Jeffrey K. Thompson, Matthew R. Peterson, and Ralph D. Freeman. "Single-neuron activity and tissue oxygenation in the cerebral cortex". In: Science 299.5609 (Feb. 2003), pp. 1070-1073. issn: 00368075. doi: 10.1126/science. 1079220.

[61] Richard D. Hoge, Jeff Atkinson, Brad Gill, Gérard R. Crelier, Sean Marrett, and G. Bruce Pike. "Linear coupling between cerebral blood flow and oxygen consumption in activated human cortex". In: Proceedings of the National Academy of Sciences of the United States of America 96.16 (Aug. 1999), pp. 9403-9408. issn: 00278424. doi: 10.1073/pnas. 96.16.9403.

[62] Grant R.J. Gordon, Hyun B. Choi, Ravi L. Rungta, Graham C.R. EllisDavies, and Brian A. MacVicar. "Brain metabolism dictates the polarity of astrocyte control over arterioles". In: Nature 456.7223 (Dec. 2008), pp. 745-750. issn: 14764687. doi: 10.1038/nature07525.

[63] Mark A. Mintun, Brian N. Lundstrom, Abraham Z. Snyder, Andrei G. Vlassenko, Gordon L. Shulman, and Marcus E. Raichle. "Blood flow and oxygen delivery to human brain during functional activity: Theoretical modeling and experimental data". In: Proceedings of the $\mathrm{Na}$ tional Academy of Sciences of the United States of America 98.12 (June 2001), pp. 6859-6864. issn: 00278424. doi: 10 .1073/pnas . 111164398.

[64] William J. Powers, Irl B. Hirsch, and Philip E. Cryer. "Effect of stepped hypoglycemia on regional cerebral blood flow response to physiological brain activation". In: American Journal of Physiology - Heart and Circulatory Physiology 270.2 39-2 (1996). issn: 03636135. doi: 10.1152/ajpheart.1996.270.2.h554.

[65] Tilo Wolf, Ute Lindauer, Arno Villringer, and Ulrich Dirnagl. "Excessive oxygen or glucose supply does not alter the blood flow response to somatosensory stimulation or spreading depression in rats". In: Brain Research 761.2 (July 1997), pp. 290-299. issn: 00068993. doi: 10.1016/S0006-8993(97)00354-5. 
[66] David Attwell and Costantino Iadecola. The neural basis of functional brain imaging signals. Dec. 2002. doi: 10 . 1016/S0166-2236 (02) 02264-6.

[67] Ute Lindauer, Christoph Leithner, Heike Kaasch, Benjamin Rohrer, Marco Foddis, Martina Füchtemeier, Nikolas Offenhauser, Jens Steinbrink, Georg Royl, Matthias Kohl-Bareis, and Ulrich Dirnagl. "Neurovascular Coupling in Rat Brain Operates Independent of Hemoglobin Deoxygenation”. In: Journal of Cerebral Blood Flow \& Metabolism 30.4 (Apr. 2010), pp. 757-768. issn: 0271-678X. doi: 10 . 1038/jcbfm . 2009 . 259. url: http: //journals . sagepub.com/doi / $10.1038 / j \mathrm{cbfm} .2009 .259$.

[68] Clare Howarth, Brad Sutherland, Hyun B. Choi, Chris Martin, Barbara Lykke Lind, Lila Khennouf, Jeffrey M. Ledue, Janelle M.P. Pakan, Rebecca W.Y. Ko, Graham Ellis-Davies, Martin Lauritzen, Nicola R. Sibson, Alastair M. Buchan, and Brian A. MacVicar. "A critical role for astrocytes in hypercapnic vasodilation in brain". In: Journal of Neuroscience 37.9 (Mar. 2017), pp. 2403-2414. issn: 15292401. doi: 10.1523/JNEUROSCI . 0005-16.2016.

[69] Alexander V. Gourine, Vitaliy Kasymov, Nephtali Marina, Feige Tang, Melina F. Figueiredo, Samantha Lane, Anja G. Teschemacher, K. Michael Spyer, Karl Deisseroth, and Sergey Kasparov. "Astrocytes control breathing through $\mathrm{pH}$-dependent release of ATP”. In: Science 329.5991 (July 2010), pp. 571-575. issn: 00368075. doi: 10 . 1126/ science.1190721.

[70] Jens Astrup, Dieter Heuser, Niels A. Lassen, Bengt Nilsson, Karin Norberg, and Bo K. Siesjö. "Evidence Against $\mathrm{H}+$ and $\mathrm{K}+$ as main Factors for the Control of Cerebral Blood Flow: A Microelectrode Study”. In: John Wiley \& Sons, Ltd, May 2008, pp. 313-337. doi: 10 . 1002/9780470720370 . ch16. url: http : // doi . wiley . com/10 . 1002/9780470720370. ch16.

[71] E. Pinard, E. Tremblay, Y. Ben-Ari, and J. Seylaz. "Blood flow compensates oxygen demand in the vulnerable ca3 region of the hippocampus during kainate-induced seizures". In: Neuroscience 13.4 (1984), pp. 1039-1049. issn: 03064522. doi: 10 . 1016 / $0306-$ 4522 (84) 90287-2.

[72] Tristan R. Hollyer, Luca Bordoni, Birgitte S. Kousholt, Judith van Luijk, Merel Ritskes-Hoitinga, and Leif Østergaard. "The evidence for the physiological effects of lactate on the cerebral microcirculation: a systematic review". In: Journal of Neurochemistry 148.6 (Mar. 2019), pp. 712-730. issn: 14714159. doi: 10.1111/jnc. 14633. 
[73] Eric A Newman. "Functional hyperemia and mechanisms of neurovascular coupling in the retinal vasculature". In: Journal of Cerebral Blood Flow \& Metabolism 33.11 (2013), pp. 1685-1695. doi: 10. 1038/jcbfm. 2013 . 145. url: http://www.ncbi.nlm.nih.gov/pmc/ articles/PMC3824187/.

[74] S. Cockcroft. Signalling roles of mammalian phospholipase D1 and D2. 2001. doi: 10.1007/PL00000805.

[75] Clotilde Lecrux, Xavier Toussay, Ara Kocharyan, Priscilla Fernandes, Sujay Neupane, Maxime Levesque, Fabrice Plaisier, Amir Shmuel, Bruno Cauli, and Edith Hamel. "Pyramidal neurons are "neurogenic hubs" in the neurovascular coupling response to whisker stimulation." In: $J$ Neurosci 31.27 (July 2011), pp. 9836-9847. issn: 15292401. doi: 10.1523/JNEUROSCI . 4943-10.2011.

[76] Alexandre Lacroix, Xavier Toussay, Eitan Anenberg, Clotilde Lecrux, Nerea Ferreiros, Anastassios Karagiannis, Fabrice Plaisier, Patrick Chausson, Frederic Jarlier, Sean A Burgess, Elizabeth M C Hillman, Irmgard Tegeder, Timothy H Murphy, Edith Hamel, and Bruno Cauli. "COX-2-Derived Prostaglandin E2 Produced by Pyramidal Neurons Contributes to Neurovascular Coupling in the Rodent Cerebral Cortex." In: $J$ Neurosci 35.34 (Aug. 2015), pp. 11791-11810. issn: 15292401. doi: 10.1523/JNEUROSCI. 0651-15.2015.

[77] C. Lecrux and E. Hamel. Neuronal networks and mediators of cortical neurovascular coupling responses in normal and altered brain states. Oct. 2016. doi: 10.1098/rstb.2015.0350.

[78] K Niwa, E Araki, S G Morham, M E Ross, and C Iadecola. "Cyclooxygenase-2 contributes to functional hyperemia in whiskerbarrel cortex.” In: $J$ Neurosci 20.2 (Jan. 2000), pp. 763-770. issn: 1529-2401 (Electronic); 0270-6474 (Linking).

[79] Richard J Davis, Colin E Murdoch, Mozam Ali, Stuart Purbrick, Rivka Ravid, Gordon S Baxter, Nick Tilford, Robert L G Sheldrick, Kenneth L Clark, and Robert A Coleman. "EP4 prostanoid receptor-mediated vasodilatation of human middle cerebral arteries." In: $\mathrm{Br} J$ Pharmacol 141.4 (Feb. 2004), pp. 580-585. issn: 0007-1188 (Print); 00071188 (Linking). doi: 10.1038/sj . bjp. 0705645.

[8o] Anusha Mishra, James P Reynolds, Yang Chen, Alexander V Gourine, Dmitri A Rusakov, and David Attwell. "Astrocytes mediate neurovascular signaling to capillary pericytes but not to arterioles." In: Nat Neurosci 19.12 (Dec. 2016), pp. 1619-1627. issn: 1546-1726 (Electronic); 1097-6256 (Linking). doi: 10.1038/nn. 4428. 
[81] Bruno Cauli, Xin-Kang Tong, Armelle Rancillac, Nella Serluca, Bertrand Lambolez, Jean Rossier, and Edith Hamel. "Cortical GABA interneurons in neurovascular coupling: relays for subcortical vasoactive pathways." In: $J$ Neurosci 24.41 (2004), pp. 8940-8949. issn: 1529-2401. doi: 10.1523/JNEUROSCI . 3065-04 . 2004.

[82] Armelle Rancillac, Jean Rossier, Manon Guille, Xin-Kang Tong, Helene Geoffroy, Christian Amatore, Stephane Arbault, Edith Hamel, and Bruno Cauli. "Glutamatergic Control of Microvascular Tone by Distinct GABA Neurons in the Cerebellum." In: $J$ Neurosci 26.26 (June 2006), pp. 6997-7006. issn: 1529-2401. doi: 10 . $1523 /$ JNEUROSCI . 5515-05.2006.

[83] U Lindauer, D Megow, H Matsuda, and U Dirnagl. "Nitric oxide: a modulator, but not a mediator, of neurovascular coupling in rat somatosensory cortex.” In: Am J Physiol 277.2 (Aug. 1999), H799-811. issn: 0002-9513. doi: 10.1152/ajpheart.1999.277.2.H799.

[84] Hiroki Taniguchi. "Genetic dissection of GABAergic neural circuits in mouse neocortex.” In: Front Cell Neurosci 8 (2014), p. 8. issn: 16625102 (Print); 1662-5102 (Linking). doi: 10.3389/fncel . 2014.00008.

[85] Patrick S. Hosford and Alexander V. Gourine. "What is the key mediator of the neurovascular coupling response?" In: Neuroscience \& Biobehavioral Reviews 96 (Jan. 2019), pp. 174-181. issn: 01497634. doi: 10 . 1016 / j . neubiorev . 2018 . 11 . 011. url: https : / / linkinghub . elsevier . com/retrieve / pii / S0149763418306158\% 20https : / / www . sciencedirect . com / science / article / pii / S0149763418306158.

[86] Henry Markram, Maria Toledo-Rodriguez, Yun Wang, Anirudh Gupta, Gilad Silberberg, and Caizhi Wu. "Interneurons of the neocortical inhibitory system." In: Nat Rev Neurosci 5.10 (Oct. 2004), pp. 793-807. issn: 1471-003X. doi: 10.1038/nrn1519.

[87] T L Yaksh, J Y Wang, and V L Go. "Cortical vasodilatation produced by vasoactive intestinal polypeptide (VIP) and by physiological stimuli in the cat." In: Journal of cerebral blood flow and metabolism : official journal of the International Society of Cerebral Blood Flow and Metabolism 7.3 (June 1987), pp. 315-26. issn: 0271-678. doi: 10.1038/jcbfm . 1987 . 69. url: http: //www . ncbi.nlm . nih.gov/ pubmed/3108270.

[88] Hana Uhlirova, Kivilcim Kilic, Peifang Tian, Martin Thunemann, Michele Desjardins, Payam A Saisan, Sava Sakadzic, Torbjorn V Ness, Celine Mateo, Qun Cheng, Kimberly L Weldy, Florence Razoux, Matthieu Vandenberghe, Jonathan A Cremonesi, Christopher Gl Ferri, Krystal Nizar, Vishnu B Sridhar, Tyler C Steed, Maxim Abashin, Yeshaiahu Fainman, Eliezer Masliah, Srdjan Djurovic, Ole A 
Andreassen, Gabriel A Silva, David A Boas, David Kleinfeld, Richard B Buxton, Gaute T Einevoll, Anders M Dale, and Anna Devor. "Cell type specificity of neurovascular coupling in cerebral cortex." In: Elife 5 (2016). issn: 2050-084X (Electronic); 2050-084X (Linking). doi: 10.7554/eLife. 14315.

[89] R Abounader, J G Villemure, and E Hamel. "Characterization of neuropeptide $\mathrm{Y}$ (NPY) receptors in human cerebral arteries with selective agonists and the new Y1 antagonist BIBP 3226.” In: British journal of pharmacology 116.4 (Oct. 1995), pp. 2245-50. issn: 0007-1188. url: http : / / www . ncbi . nlm . nih . gov/pubmed / 8564255\%20http : / / www . pubmedcentral . nih . gov/articlerender . fcgi ? artid = PMC1908978.

[90] Ara Kocharyan, Priscilla Fernandes, Xin-Kang Tong, Elvire Vaucher, and Edith Hamel. "Specific subtypes of cortical GABA interneurons contribute to the neurovascular coupling response to basal forebrain stimulation." In: J Cereb Blood Flow Metab 28.2 (Feb. 2008), pp. 221-231. issn: 0271-678. doi: 10.1038/sj . jcbfm. 9600558.

[91] J B Long, D D Rigamonti, K Dosaka, J M Kraimer, and A MartinezArizala. "Somatostatin causes vasoconstriction, reduces blood flow and increases vascular permeability in the rat central nervous system." In: Journal of Pharmacology and Experimental Therapeutics 260.3 (1992).

[92] L Lee, L Boorman, E Glendenning, C Christmas, P Sharp, P Redgrave, O Shabir, E Bracci, J Berwick, and C Howarth. "Key Aspects of Neurovascular Control Mediated by Specific Populations of Inhibitory Cortical Interneurons." In: Cerebral cortex (New York, N.Y. : 1991) (Nov. 2019). issn: 1460-2199. doi: 10 . 1093 / cercor / bhz251. url: http://www.ncbi.nlm.nih.gov/pubmed/31746324.

[93] L Bao, J Kopp, X Zhang, Z Q Xu, L F Zhang, H Wong, J Walsh, and T Hökfelt. "Localization of neuropeptide Y Y1 receptors in cerebral blood vessels." In: Proceedings of the National Academy of Sciences of the United States of America 94.23 (Nov. 1997), pp. 12661-6. issn: 0027-8424. url: http://www.ncbi.nlm.nih.gov/pubmed/9356506\% 20http : / / ww . pubmedcentral . nih . gov/articlerender . fcgi? artid=PMC25075.

[94] Roger Abounader, Ahmed Elhusseiny, Zvi Cohen, André Olivier, Danica Stanimirovic, Rémi Quirion, and Edith Hamel. "Expression of Neuropeptide Y Receptors mRNA and Protein in Human Brain Vessels and Cerebromicrovascular Cells in Culture". In: Journal of Cerebral Blood Flow \& Metabolism 19.2 (Feb. 1999), pp. 155-163. issn: 0271-678. doi: 10 . 1097 / 00004647 - 199902000-00007. url: http : 
//www.ncbi.nlm.nih.gov/pubmed/10027771\%20http://journals . sagepub.com/doi/10.1097/00004647-199902000-00007.

[95] Cheryl M. J. Tan, Peregrine Green, Nidi Tapoulal, Adam J. Lewandowski, Paul Leeson, and Neil Herring. "The Role of Neuropeptide Y in Cardiovascular Health and Disease". In: Frontiers in Physiology 9 (Sept. 2018), p. 1281. issn: 1664-042. doi: 10 . 3389 / fphys. 2018.01281. url: https://www . frontiersin.org/article/ 10.3389/fphys.2018.01281/full.

[96] Alberto L Vazquez, Mitsuhiro Fukuda, and Seong-Gi Kim. "Inhibitory Neuron Activity Contributions to Hemodynamic Responses and Metabolic Load Examined Using an Inhibitory Optogenetic Mouse Model.” In: Cereb Cortex 28.11 (Nov. 2018), pp. 4105-4119. issn: 1460-2199. doi: 10.1093/cercor/bhy225.

[97] David G Rosenegger, Cam Ha T Tran, Jackie I Wamsteeker Cusulin, and Grant R Gordon. "Tonic Local Brain Blood Flow Control by Astrocytes Independent of Phasic Neurovascular Coupling". In: The Journal of Neuroscience 35.39 (2015), p. 13463. url: http : / / www . jneurosci.org/content/35/39/13463. abstract.

[98] J. A. Filosa, H. W. Morrison, J. A. Iddings, W. Du, and K. J. Kim. Beyond neurovascular coupling, role of astrocytes in the regulation of vascular tone. May 2016. doi: 10 .1016/j . neuroscience. 2015. 03.064 .

[99] Jennifer A. Iddings, Ki Jung Kim, Yiqiang Zhou, Haruki Higashimori, and Jessica A. Filosa. "Enhanced parenchymal arteriole tone and astrocyte signaling protect neurovascular coupling mediated parenchymal arteriole vasodilation in the spontaneously hypertensive rat". In: Journal of Cerebral Blood Flow and Metabolism 35.7 (July 2015), pp. 1127-1136. issn: 15597016. doi: 10 . 1038/ jcbfm . 2015 . 31. url: http : / / www . ncbi . nlm . nih . gov/ pubmed / 25757753\% 20http : / / www . pubmedcentral . nih . gov / articlerender . fcgi ? artid = PMC4640269.

[100] Ki Jung Kim, Jennifer A. Iddings, Javier E. Stern, Víctor M. Blanco, Deborah Croom, Sergei A. Kirov, and Jessica A. Filosa. "Astrocyte contributions to flow/pressure-evoked parenchymal arteriole vasoconstriction". In: Journal of Neuroscience 35.21 (May 2015), pp. 8245-8257. issn: 15292401. doi: 10.1523/ JNEUROSCI . 4486-14. 2015. url: http : / /www . embase. $\mathrm{com} /$ search/results ? subaction= viewrecord\&from=export\&id=L604696584\%20http://dx.doi . org/ 10.1523/JNEUROSCI . 4486-14. 2015.

[101] Narges Bazargani and David Attwell. "Astrocyte calcium signaling: the third wave". In: Nat Neurosci 19.2 (2016), pp. 182-189. issn: 1097-6256. doi: 10.1038/nn. 4201. 
[102] Eric A Newman. "Glial cell regulation of neuronal activity and blood flow in the retina by release of gliotransmitters." In: Philos Trans $R$ Soc Lond B Biol Sci 370.1672 (July 2015). issn: 1471-2970 (Electronic); 0962-8436 (Linking). doi: 10.1098/rstb. 2014.0195.

[103] Brian A MacVicar and Eric A Newman. "Astrocyte regulation of blood flow in the brain." In: Cold Spring Harb Perspect Biol 7.5 (Mar. 2015). issn: 1943-0264 (Electronic); 1943-0264 (Linking). doi: 10 . 1101/cshperspect.a020388.

[104] Monica R Metea and Eric A Newman. "Glial cells dilate and constrict blood vessels: a mechanism of neurovascular coupling." In: $J$ Neurosci 26.11 (Mar. 2006), pp. 2862-2870. issn: 1529-2401 (Electronic); 0270-6474 (Linking). doi: 10 . 1523/ JNEUROSCI . 4048- 05 . 2006.

[105] Sean J Mulligan and Brian A MacVicar. "Calcium transients in astrocyte endfeet cause cerebrovascular constrictions." In: Nature 431.7005 (Sept. 2004), pp. 195-199. issn: 1476-4687 (Electronic); 0028-0836 (Linking). doi: 10.1038/nature02827.

[106] Grant R J Gordon, Clare Howarth, and Brian A MacVicar. "Bidirectional control of arteriole diameter by astrocytes." In: Exp Physiol 96.4 (Apr. 2011), pp. 393-399. issn: 1469-445X (Electronic); 09580670 (Linking). doi: 10.1113/expphysiol.2010.053132.

[107] A Lange, D Gebremedhin, J Narayanan, and D Harder. "20Hydroxyeicosatetraenoic acid-induced vasoconstriction and inhibition of potassium current in cerebral vascular smooth muscle is dependent on activation of protein kinase C." In: $J$ Biol Chem 272.43 (1997), pp. 27345-27352. issn: 0021-9258.

[108] D Gebremedhin, A R Lange, T F Lowry, M R Taheri, E K Birks, A G Hudetz, J Narayanan, J R Falck, H Okamoto, R J Roman, K Nithipatikom, W B Campbell, and D R Harder. "Production of 20HETE and its role in autoregulation of cerebral blood flow." In: Circ Res 87.1 (July 2000), pp. 60-65. issn: 0oo9-7330.

[109] Wei Sun, Evan McConnell, Jean-Francois Pare, Qiwu Xu, Michael Chen, Weiguo Peng, Ditte Lovatt, Xiaoning Han, Yoland Smith, and Maiken Nedergaard. "Glutamate-Dependent Neuroglial Calcium Signaling Differs Between Young and Adult Brain”. In: Science (New York, N.Y.) 339.6116 (2013), pp. 197-200. doi: 10.1126/science. 1226740. url: http : / / www . ncbi .nlm . nih.gov/pmc/articles / PMC3569008/. 
[110] Daniel E Bonder and Ken D McCarthy. "Astrocytic Gq-GPCR-linked IP3R-dependent $\mathrm{Ca} 2+$ signaling does not mediate neurovascular coupling in mouse visual cortex in vivo." In: $J$ Neurosci 34.39 (Sept. 2014), pp. 13139-13150. issn: 1529-2401 (Electronic); 0270-6474 (Linking). doi: 10.1523/JNEUROSCI .2591-14.2014.

[111] Thomas A Longden, Fabrice Dabertrand, Masayo Koide, Albert L Gonzales, Nathan R Tykocki, Joseph E Brayden, David Hill-Eubanks, and Mark T Nelson. "Capillary K+-sensing initiates retrograde hyperpolarization to increase local cerebral blood flow". In: Nature Neuroscience 20.5 (May 2017), pp. 717-726. issn: 1097-6256. doi: 10.1038/ nn. 4533. url: http://www nature . com/articles/nn. 4533.

[112] Hana Uhlirova, Kivilcim Kilic, Peifang Tian, Sava Sakadzic, Louis Gagnon, Martin Thunemann, Michele Desjardins, Payam A Saisan, Krystal Nizar, Mohammad A Yaseen, Donald J Jr Hagler, Matthieu Vandenberghe, Srdjan Djurovic, Ole A Andreassen, Gabriel A Silva, Eliezer Masliah, David Kleinfeld, Sergei Vinogradov, Richard B Buxton, Gaute T Einevoll, David A Boas, Anders M Dale, and Anna Devor. "The roadmap for estimation of cell-type-specific neuronal activity from non-invasive measurements." In: Philos Trans $R$ Soc Lond B Biol Sci 371.1705 (2016). issn: 1471-2970 (Electronic); 0962-8436 (Linking). doi: 10.1098/rstb.2015.0356.

[113] Michael B. Krawchuk, Catherine F. Ruff, Xiaoling Yang, Sarah E. Ross, and Alberto L. Vazquez. "Optogenetic assessment of VIP, PV, SOM and NOS inhibitory neuron activity and cerebral blood flow regulation in mouse somato-sensory cortex". In: Journal of Cerebral Blood Flow and Metabolism 40.7 (July 2019). issn: 15597016. doi: 10.1177/0271678X19870105. url: https ://pubmed.ncbi.nlm.nih. gov/31418628/.

[114] Robert W Brown, Yu-Chung N Cheng, E Mark Haacke, Michael R Thompson, and Ramesh Venkatesan. Magnetic resonance imaging : physical principles and sequence design. English. 2014. url: http: //site.ebrary.com/id/10861211.

[115] Richard B. Buxton. "The physics of functional magnetic resonance imaging (fMRI)". In: Reports on Progress in Physics 76.9 (2013). issn: 00344885. doi: 10.1088/0034-4885/76/9/096601. url: https : //pubmed.ncbi.nlm.nih.gov/24006360/.

[116] Donald W McRobbie, Elizabeth A Moore, and Martin J Graves. MRI from Picture to Proton. English. West Nyack, 2017. url: https : / / public. ebookcentral . proquest. com/choice/publicfullrecord. aspx?p=4829805. 
[117] Sean Novak. File:Normal axial T2-weighted MR image of the brain.jpg - Wikimedia Commons. url: https : / / commons . wikimedia _org/wiki / File : Normal_axial_ T2 - weighted_MR_ image_of_the_brain.jpg (visited on 06/12/2020).

[118] K. K. Kwong, J. W. Belliveau, D. A. Chesler, I. E. Goldberg, R. M. Weisskoff, B. P. Poncelet, D. N. Kennedy, B. E. Hoppel, M. S. Cohen, R. Turner, H. M. Cheng -, T. J. Brady, and B. R. Rosen. "Dynamic magnetic resonance imaging of human brain activity during primary sensory stimulation". In: Proceedings of the National Academy of Sciences of the United States of America 89.12 (June 1992), pp. 5675-5679. issn: 00278424. doi: 10.1073/pnas .89.12. 5675.

[119] Linus Pauling and Charles D. Coryell. "The Magnetic Properties and Structure of Hemoglobin, Oxyhemoglobin and Carbonmonoxyhemoglobin". In: Proceedings of the National Academy of Sciences 22.4 (Apr. 1936), pp. 210-216. issn: 0027-8424. doi: 10.1073/pnas . 22 . 4.210.

[120] Keith R. Thulborn, John C. Waterton, Paul M. Matthews, and George K. Radda. "Oxygenation dependence of the transverse relaxation time of water protons in whole blood at high field". In: BBA - General Subjects 714.2 (Feb. 1982), pp. 265-270. issn: 03044165. doi: 10.1016/ 0304-4165 (82) 90333-6.

[121] A. M. Blamire, S. Ogawa, K. Ugurbil, D. Rothman, G. McCarthy, J. M. Ellermann, F. Hyder, Z. Rattner, and R. G. Shulman. "Dynamic mapping of the human visual cortex by high-speed magnetic resonance imaging”. In: Proceedings of the National Academy of Sciences of the United States of America 89.22 (Nov. 1992), pp. 11069-11073. issn: 00278424. doi: 10.1073/pnas .89.22.11069.

[122] Jens Frahm, Harald Bruhn, Klaus $\square$ Dietmar $\square$ D Merboldt, and Wolfgang Hänicke. "Dynamic MR imaging of human brain oxygenation during rest and photic stimulation". In: Journal of Magnetic Resonance Imaging 2.5 (Sept. 1992), pp. 501-505. issn: 15222586. doi: 10.1002/jmri.1880020505.

[123] R. Turner, P. Jezzard, H. Wen, K. K. Kwong, D. Le Bihan, T. Zeffiro, and R. S. Balaban. "Functional mapping of the human visual cortex at 4 and 1.5 tesla using deoxygenation contrast EPI". In: Magnetic Resonance in Medicine 29.2 (1993), pp. 277-279. issn: 15222594. doi: $10.1002 / \mathrm{mrm} .1910290221$.

[124] S. Ogawa, D. W. Tank, R. Menon, J. M. Ellermann, S. G. Kim, H. Merkle, and K. Ugurbil. "Intrinsic signal changes accompanying sensory stimulation: Functional brain mapping with magnetic resonance imaging”. In: Proceedings of the National Academy of Sciences of 
the United States of America 89.13 (July 1992), pp. 5951-5955. issn: 00278424. doi: 10.1073/pnas .89.13.5951.

[125] R B Buxton. "The elusive initial dip." eng. In: NeuroImage $13.6 \mathrm{Pt}$ 1 (June 2001), pp. 953-958. issn: 1053-8119 (Print). doi: $10.1006 /$ nimg. 2001.0814.

[126] Xiaoping Hu and Essa Yacoub. The story of the initial dip in fMRI. Aug. 2012. doi: $10.1016 / \mathrm{j}$. neuroimage . 2012 .03 .005. url: /pmc/ articles/PMC3389272/?report=abstract\%20https : //www . ncbi . nlm.nih.gov/pmc/articles/PMC3389272/.

[127] Timothy L. Davis, Kenneth K. Kwong, Robert M. Weisskoff, and Bruce R. Rosen. "Calibrated functional MRI: Mapping the dynamics of oxidative metabolism”. In: Proceedings of the National Academy of Sciences of the United States of America 95.4 (Feb. 1998), pp. 1834-1839. issn: 00278424. doi: 10.1073/pnas .95.4.1834.

[128] Nicholas P. Blockley, Valerie E.M. Griffeth, Aaron B. Simon, and Richard B. Buxton. "A review of calibrated blood oxygenation leveldependent (BOLD) methods for the measurement of task-induced changes in brain oxygen metabolism". In: NMR in Biomedicine 26.8 (Aug. 2013), pp. 987-1003. issn: 09523480. doi: 10.1002/nbm. 2847. url: https://pubmed.ncbi.nlm.nih.gov/22945365/.

[129] Eric C. Wong, Richard B. Buxton, and Lawrence R. Frank. "Implementation of quantitative perfusion imaging techniques for functional brain mapping using pulsed arterial spin labeling". In: $N M R$ in Biomedicine 10.4-5 (1997), pp. 237-249. issn: 09523480. doi: 10. 1002/ (SICI) 1099-1492 (199706/08) 10:4/5<237: : AID-NBM475>3. $0 . \mathrm{CO} ; 2-\mathrm{X}$.

[130] Eric C. Wong, Richard B. Buxton, and Lawrence R. Frank. "Quantitative imaging of perfusion using a single subtraction (QUIPSS and QUIPSS II)”. In: Magnetic Resonance in Medicine 39.5 (May 1998), pp. 702-708. issn: 07403194. doi: 10.1002/mrm. 1910390506.

[131] John A. Detre, John S. Leigh, Donald S. Williams, and Alan P. Koretsky. "Perfusion imaging". In: Magnetic Resonance in Medicine 23.1 (1992), pp. 37-45. issn: 15222594. doi: 10.1002/mrm. 1910230106.

[132] Hanzhang Lu, Jun Hua, and Peter C.M. van Zijl. "Noninvasive functional imaging of cerebral blood volume with vascular-spaceoccupancy (VASO) MRI”. In: NMR in Biomedicine 26.8 (Aug. 2013), pp. 932-948. issn: 09523480. doi: 10.1002/nbm. 2905. 
[133] Laurentius Huber, Dimo Ivanov, Steffen N. Krieger, Markus N. Streicher, Toralf Mildner, Benedikt A. Poser, Harald E. Möller, and Robert Turner. "Slab-selective, BOLD-corrected VASO at 7 tesla provides measures of cerebral blood volume reactivity with high signalto-noise ratio". In: Magnetic Resonance in Medicine 72.1 (2014), pp. 137-148. issn: 15222594. doi: 10.1002/mrm. 24916.

[134] Laurentius Huber, Jozien Goense, Aneurin J. Kennerley, Dimo Ivanov, Steffen N. Krieger, Jöran Lepsien, Robert Trampel, Robert Turner, and Harald E. Möller. "Investigation of the neurovascular coupling in positive and negative BOLD responses in human brain at 7 T.” In: NeuroImage 97 (Aug. 2014), pp. 349-362. issn: 1053-8119. doi: $10.1016 / j$. neuroimage .2014 .04 .022 .

[135] Anna Devor, Sava Sakadžić, Vivek J Srinivasan, Mohammad A Yaseen, Krystal Nizar, Payam A Saisan, Peifang Tian, Anders M Dale, Sergei A Vinogradov, Maria Angela Franceschini, and David A Boas. "Frontiers in Optical Imaging of Cerebral Blood Flow and Metabolism”. In: Journal of Cerebral Blood Flow \& Metabolism 32.7 (July 2012), pp. 1259-1276. issn: 0271-678X. doi: $10.1038 / \mathrm{jcbfm}$. 2011 . 195. url: http : / / journals . sagepub . com / doi / 10 . 1038 / jcbfm.2011.195.

[136] Anna Devor, Istvan Ulbert, Andrew K. Dunn, Suresh N. Narayanan, Stephanie R. Jones, Mark L. Andermann, David A. Boas, and Anders M. Dale. "Coupling of the cortical hemodynamic response to cortical and thalamic neuronal activity." In: Proc Natl Acad Sci U S A 102.10 (Mar. 2005), pp. 3822-3827. issn: 0027-8424 (Print); 0027-8424 (Linking). doi: 10.1073/pnas . 0407789102.

[137] Patrick J. Drew, Andy Y. Shih, and David Kleinfeld. "Fluctuating and sensory-induced vasodynamics in rodent cortex extend arteriole capacity". In: Proceedings of the National Academy of Sciences of the United States of America 108.20 (May 2011), pp. 8473-8478. issn: 00278424. doi: 10.1073/pnas .1100428108. url: http://www. ncbi. nlm . nih. gov/pubmed/21536897\%20http: / / ww . pubmedcentral . nih.gov/articlerender. fcgi?artid=PMC3100929.

[138] Amiram Grinvald, Edmund Lieke, Ron D. Frostig, Charles D. Gilbert, and Torsten N. Wiesel. "Functional architecture of cortex revealed by optical imaging of intrinsic signals". In: Nature 324.6095 (Nov. 1986), pp. 361-364. issn: 00280836. doi: 10.1038/324361a0.

[139] Andy Y. Shih, Jonathan D. Driscoll, Patrick J. Drew, Nozomi Nishimura, Chris B. Schaffer, and David Kleinfeld. Two-photon microscopy as a tool to study blood flow and neurovascular coupling in the rodent brain. July 2012. doi: 10.1038/j cbfm . 2011 .196. url: http://journals.sagepub.com/doi/10.1038/jcbfm.2011.196. 
[140] Andy Y. Shih, Patrick J. Drew, and David Kleinfeld. "Imaging vasodynamics in the Awake mouse brain with two-photon microscopy". In: Neuromethods 88 (2014), pp. 55-73. issn: 19406045. doi: 10.1007/ 978-1-4939-0724-3_4.

[141] A. Villringer, J. Planck, C. Hock, L. Schleinkofer, and U. Dirnagl. "Near infrared spectroscopy (NIRS): A new tool to study hemodynamic changes during activation of brain function in human adults". In: Neuroscience Letters 154.1-2 (May 1993), pp. 101-104. issn: 03043940. doi: 10.1016/0304-3940 (93) 90181-J.

[142] Yoko Hoshi and Mamoru Tamura. "Detection of dynamic changes in cerebral oxygenation coupled to neuronal function during mental work in man”. In: Neuroscience Letters 150.1 (Feb. 1993), pp. 5-8. issn: 03043940. doi: 10.1016/0304-3940 (93) 90094-2.

[143] F. Jöbsis. "Noninvasive, infrared monitoring of cerebral and myocardial oxygen sufficiency and circulatory parameters". In: Science 198.4323 (Dec. 1977), pp. 1264-1267. issn: 0036-8075. doi: 10.1126/ science. 929199.

[144] P Ellen Grant, Nadege Roche-Labarbe, Andrea Surova, George Themelis, Juliette Selb, Elizabeth K Warren, Kalpathy S Krishnamoorthy, David A Boas, and Maria Angela Franceschini. "Increased Cerebral Blood Volume and Oxygen Consumption in Neonatal Brain Injury". In: Journal of Cerebral Blood Flow \& Metabolism 29.10 (Oct. 2009), pp. 1704-1713. issn: 0271-678X. doi: 10 . 1038/ jcbfm . 2009 . 90. url: http : / journals . sagepub . com/doi / 10 . 1038/jcbfm. 2009.90.

[145] S. Lloyd-Fox, A. Blasi, and C. E. Elwell. Illuminating the developing brain: The past, present and future of functional near infrared spectroscopy. Feb. 2010. doi: 10.1016/j . neubiorev . 2009.07.008.

[146] A. C. Dieler, S. V. Tupak, and A. J. Fallgatter. "Functional nearinfrared spectroscopy for the assessment of speech related tasks". In: Brain and Language 121.2 (May 2012), pp. 90-109. issn: 0093934X. doi: $10.1016 / \mathrm{j}$. bandl .2011 .03 .005 .

[147] Edward S. Boyden, Feng Zhang, Ernst Bamberg, Georg Nagel, and Karl Deisseroth. "Millisecond-timescale, genetically targeted optical control of neural activity". In: Nature Neuroscience 8.9 (Aug. 2005), pp. 1263-1268. issn: 10976256. doi: 10 . 1038/nn1525. url: https : //www.nature.com/articles/nn1525.

[148] Lief Fenno, Ofer Yizhar, and Karl Deisseroth. "The Development and Application of Optogenetics". In: Annual Review of Neuroscience 34.1 (July 2011), pp. 389-412. issn: 0147-006X. doi: 10 . $1146 /$ annurev-neuro-061010-113817. 
[149] Georg Nagel, Tanjef Szellas, Wolfram Huhn, Suneel Kateriya, Nona Adeishvili, Peter Berthold, Doris Ollig, Peter Hegemann, and Ernst Bamberg. "Channelrhodopsin-2, a directly light-gated cation-selective membrane channel". In: Proceedings of the National Academy of Sciences of the United States of America 100.SUPPL. 2 (Nov. 2003), pp. 13940-13945. issn: 00278424. doi: 10.1073/pnas. 1936192100.

[150] Diana H. Lim and Jeffrey LeDue. "What Is Optogenetics and How Can We Use It to Discover More About the Brain?" In: Frontiers for Young Minds 5 (Sept. 2017). doi: 10.3389/frym. 2017.00051.

[151] Henning M. Reimann and Thoralf Niendorf. "The (Un)Conscious Mouse as a Model for Human Brain Functions: Key Principles of Anesthesia and Their Impact on Translational Neuroimaging". In: Frontiers in Systems Neuroscience 14 (May 2020), p. 8. issn: 16625137. doi: 10.3389 /fnsys . 2020.00008.

[152] Kazuto Masamoto and Iwao Kanno. "Anesthesia and the quantitative evaluation of neurovascular coupling.” In: J Cereb Blood Flow Metab 32.7 (July 2012), pp. 1233-1247. issn: 1559-7016 (Electronic); 0271678X (Linking). doi: 10.1038/jcbfm. 2012.50.

[153] Yu-Rong Gao, Yuncong Ma, Qingguang Zhang, Aaron T. Winder, Zhifeng Liang, Lilith Antinori, Patrick J. Drew, and Nanyin Zhang. "Time to wake up: Studying neurovascular coupling and brain-wide circuit function in the un-anesthetized animal". In: NeuroImage 153 (June 2017), pp. 382-398. issn: 1053-8119. doi: 10 . 1016 / J . NEUROIMAGE . 2016.11.069. url: https : / www . sciencedirect. com/ science/article/pii/S1053811916306875?via\%3Dihub\#bib202\% 20https : / / ww . sciencedirect . com / science / article / pii / S1053811916306875?via\%7B\%5C\%25\%7D3Dihub\%7B\%5C\#\%7Dbib202.

[154] N. P. Franks and W. R. Lieb. "Molecular and cellular mechanisms of general anaesthesia". In: Nature 367.6464 (Feb. 1994), pp. 607-614. issn: 0028-0836. doi: 10.1038/367607a0. url: http://www. nature. com/articles/367607a0.

[155] Nicholas P. Franks. "General anaesthesia: from molecular targets to neuronal pathways of sleep and arousal". In: Nature Reviews Neuroscience 9.5 (May 2008), pp. 370-386. issn: 1471-003X. doi: 10 . 1038/nrn2372. url: http://www. nature. com/articles/nrn2372.

[156] Chris Martin, John Martindale, Jason Berwick, and John Mayhew. "Investigating neural-hemodynamic coupling and the hemodynamic response function in the awake rat". In: NeuroImage 32.1 (Aug. 2006), pp. 33-48. issn: 1053-8119. doi: 10 . 1016 / J . NEUROIMAGE . 2006 . 02 . 021. url: https : / /ww . sciencedirect . com/science / article/pii/S1053811906001352. 
[157] Daniil P Aksenov, Limin Li, Michael J Miller, Gheorghe Iordanescu, and Alice M Wyrwicz. "Effects of Anesthesia on BOLD Signal and Neuronal Activity in the Somatosensory Cortex". In: Journal of Cerebral Blood Flow \& Metabolism 35.11 (Nov. 2015), pp. 1819-1826. issn: 0271-678X. doi: 10 . 1038 / jcbfm . 2015 . 130. url: http : / / www . ncbi . nlm . nih . gov / pubmed / $26104288 \%$ 20http : / / www . pubmedcentral . nih . gov / articlerender . fcgi ? artid $=$ PMC4635237\%20http : / / journals . sagepub . com / doi / 10 . 1038 / jcbfm.2015.130.

[158] M. Andrea Pisauro, Neel T. Dhruv, Matteo Carandini, and Andrea Benucci. "Fast Hemodynamic Responses in the Visual Cortex of the Awake Mouse". In: Journal of Neuroscience 33.46 (Nov. 2013), pp. 18343-18351. issn: 0270-6474. doi: 10.1523/ JNEUROSCI . 213013. 2013. url: http://www.ncbi.nlm.nih.gov/pubmed/24227743\% 20http : / /ww . pubmedcentral . nih . gov/articlerender . fcgi? artid=PMC3828474.

[159] Maria Angela Franceschini, Harsha Radhakrishnan, Kiran Thakur, Weicheng Wu, Svetlana Ruvinskaya, Stefan Carp, and David A Boas. "The effect of different anesthetics on neurovascular coupling." In: Neuroimage 51.4 (July 2010), pp. 1367-1377. issn: 1095-9572 (Electronic); 1053-8119 (Print); 1053-8119 (Linking). doi: 10 . 1016/ j . neuroimage. 2010.03.060.

[160] Jaakko Paasonen, Petteri Stenroos, Raimo A Salo, Vesa Kiviniemi, and Olli Grohn. "Functional connectivity under six anesthesia protocols and the awake condition in rat brain." In: Neuroimage 172 (May 2018), pp. 9-20. issn: 1095-9572 (Electronic); 1053-8119 (Linking). doi: $10.1016 / j$. neuroimage 2018.01 .014 .

[161] Kazuto Masamoto, Mitsuhiro Fukuda, Alberto Vazquez, and SeongGi Kim. "Dose-dependent effect of isoflurane on neurovascular coupling in rat cerebral cortex". In: European Journal of Neuroscience 30.2 (July 2009), pp. 242-250. issn: 0953816X. doi: 10 . 1111/j . 1460-9568.2009.06812 .x. url: http://doi .wiley.com/10.1111/ j.1460-9568.2009.06812.x.

[162] Felix Schlegel, Aileen Schroeter, and Markus Rudin. "The hemodynamic response to somatosensory stimulation in mice depends on the anesthetic used: Implications on analysis of mouse fMRI data". In: NeuroImage 116 (Aug. 2015), pp. 40-49. issn: 10959572. doi: $10.1016 / \mathrm{j}$. neuroimage . 2015.05.013.

[163] Jozien B.M. Goense and Nikos K. Logothetis. "Neurophysiology of the BOLD fMRI Signal in Awake Monkeys". In: Current Biology 18.9 (May 2008), pp. 631-640. issn: 09609822. doi: 10 . 1016/j . cub . 2008 . 03 . 054. url: http : / / www . ncbi .nlm . nih . gov / pubmed / 
18439825\%20https : //linkinghub.elsevier.com/retrieve/pii/ S0960982208004429.

[164] U Lindauer, A Villringer, and U Dirnagl. "Characterization of CBF response to somatosensory stimulation: model and influence of anesthetics." In: Am J Physiol 264.4 Pt 2 (Apr. 1993), H1223-8. issn: 0002-9513 (Print); 0002-9513 (Linking). doi: 10 .1152/ajpheart . 1993.264.4.H1223.

[165] M Ueki, G Mies, and K A Hossmann. "Effect of alpha-chloralose, halothane, pentobarbital and nitrous oxide anesthesia on metabolic coupling in somatosensory cortex of rat." In: Acta Anaesthesiol Scand 36.4 (May 1992), pp. 318-322. issn: 0001-5172 (Print); 00015172 (Linking).

[166] K M Garrett and J Gan. "Enhancement of gamma-aminobutyric acidA receptor activity by alpha-chloralose." In: $J$ Pharmacol Exp Ther 285.2 (May 1998), pp. 680-686. issn: 0022-3565 (Print); 00223565 (Linking).

[167] Lucie A Low, Lucy C Bauer, and Brenda A Klaunberg. "Comparing the Effects of Isoflurane and Alpha Chloralose upon Mouse Physiology." In: PLoS One 11.5 (2016), e0154936. issn: 1932-6203 (Electronic); 1932-6203 (Linking). doi: 10.1371/journal pone. 0154936.

[168] Michael Vanlandewijck, Liqun He, Maarja Andaloussi Mäe, Johanna Andrae, Koji Ando, Francesca Del Gaudio, Khayrun Nahar, Thibaud Lebouvier, Bàrbara Laviña, Leonor Gouveia, Ying Sun, Elisabeth Raschperger, Markus Räsänen, Yvette Zarb, Naoki Mochizuki, Annika Keller, Urban Lendahl, and Christer Betsholtz. "A molecular atlas of cell types and zonation in the brain vasculature". In: $\mathrm{Na}$ ture 554.7693 (Feb. 2018), pp. 475-480. issn: 0028-0836. doi: 10 . 1038 / nature25739. url: http : / / www . nature . com / articles / nature25739.

[169] Alan Aderem. Systems biology: Its practice and challenges. May 2005. doi: 10.1016/j. cell.2005.04.020.

[170] Elin Nyman, Gunnar Cedersund, and Peter Strålfors. "Insulin signaling - mathematical modeling comes of age". In: Trends in Endocrinology and Metabolism 23.3 (Mar. 2012), pp. 107-115. issn: 10432760. doi: 10.1016/j.tem.2011.12.007.

[171] Gunnar Cedersund and Jacob Roll. "Systems biology: model based evaluation and comparison of potential explanations for given biological data." In: FEBS J 276.4 (Feb. 2009), pp. 903-922. issn: 17424658 (Electronic); 1742-464X (Linking). doi: 10 . 1111 / j . $1742-$ 4658.2008.06845.x. 
[172] Ruth Merkle, Bernhard Steiert, Florian Salopiata, Sofia Depner, Andreas Raue, Nao Iwamoto, Max Schelker, Helge Hass, Marvin Wäsch, Martin E. Böhm, Oliver Mücke, Daniel B. Lipka, Christoph Plass, Wolf D. Lehmann, Clemens Kreutz, Jens Timmer, Marcel Schilling, and Ursula Klingmüller. "Identification of Cell Type-Specific Differences in Erythropoietin Receptor Signaling in Primary Erythroid and Lung Cancer Cells". In: PLoS Computational Biology 12.8 (Aug. 2016). issn: 15537358. doi: 10.1371/journal . pcbi. 1005049.

[173] Edda Klipp, Wolfram Liebermeister, Christoph Wierling, and Axel Kowald. Systems Biology: A Textbook, 2nd Edition. May 2016. isbn: 978-3-527-33636-4.

[174] Darren J. Wilkinson. Stochastic modelling for quantitative description of heterogeneous biological systems. Feb. 2009. doi: 10.1038/ nrg2509.

[175] Patrick J. Rabier and Werner C. Rheinboldt. Theoretical and numerical analysis of differential-algebraic equations. Jan. 2002. doi: 10 . 1016/S1570-8659 (02) 08004-3.

[176] Leonhard Euler. Institutionum calculi integralis. Editio ter. Petropoli: Impensis Academiae Imperialis Scientiarum, 1824.

[177] Alan C Hindmarsh, Peter N Brown, Keith E Grant, Steven L Lee, Radu Serban, Dan E Shumaker, and Carol S Woodward. SUNDIALS: Suite of Nonlinear and Differential/Algebraic Equation Solvers. Tech. rep. 2005. url: https://computing.llnl.gov/projects/sundials/ toms $\% 7 \mathrm{~B} \% 5 \mathrm{C}$ _\%7Dsundials.pdf $\% 20 \mathrm{https}$ ://computing. Ilnl.gov/ projects/sundials/toms_sundials.pdf.

[178] C Runge. "Ueber die numerische Auflösung von Differentialgleichungen". In: Mathematische Annalen 46.2 (June 1895), pp. 167-178. issn: 1432-1807. doi: 10.1007/BF01446807. url: https ://doi .org/ 10.1007/BF01446807.

[179] Wilhelm Kutta. "Beitrag zur näherungsweisen Integration totaler Differentialgleichungen”. German. PhD thesis. Leipzig, 1901.

[180] Fabian Frohlich, Barbara Kaltenbacher, Fabian J Theis, and Jan Hasenauer. "Scalable Parameter Estimation for Genome-Scale Biochemical Reaction Networks.” In: PLoS Comput Biol 13.1 (Jan. 2017), e1005331. issn: 1553-7358 (Electronic); 1553-734X (Linking). doi: 10.1371/journal.pcbi.1005331.

[181] Fabian Frohlich, Fabian J Theis, Joachim O Radler, and Jan Hasenauer. "Parameter estimation for dynamical systems with discrete events and logical operations.” In: Bioinformatics 33.7 (Apr. 2017), pp. 1049-1056. issn: 1367-4811 (Electronic); 1367-4803 (Linking). doi: 10.1093/bioinformatics/btw764. 
[182] Jorge Nocedal and Stephen Wright. Numerical Optimization. Jan. 2006. isbn: 978-0-387-30303-1. doi: 10.1007/978-0-387-40065-5.

[183] Oleg Alexandrov. File:Newton optimization vs grad descent.svg Wikimedia Commons. 2007. url: https : / / commons . wikimedia . org / wiki / File : Newton_optimization_vs_grad_descent . svg (visited on 06/30/2020).

[184] Andreas Raue, Marcel Schilling, Julie Bachmann, Andrew Matteson, Max Schelke, Daniel Kaschek, Sabine Hug, Clemens Kreutz, Brian D. Harms, Fabian J. Theis, Ursula Klingmüller, and Jens Timmer. "Lessons Learned from Quantitative Dynamical Modeling in Systems Biology”. In: PLoS ONE 8.9 (Sept. 2013), e74335. issn: 19326203. doi: 10.1371 /journal . pone. 0074335.

[185] M. D. McKay, R. J. Beckman, and W. J. Conover. "A Comparison of Three Methods for Selecting Values of Input Variables in the Analysis of Output from a Computer Code". In: Technometrics 21.2 (May 1979), p. 239. issn: 00401706. doi: 10 . $2307 / 1268522$. url: https : //www. jstor.org/stable/1268522.

[186] Alejandro F Villaverde, Fabian Fröhlich, Daniel Weindl, Jan Hasenauer, and Julio R Banga. "Benchmarking optimization methods for parameter estimation in large kinetic models." eng. In: Bioinformatics (Oxford, England) 35.5 (Mar. 2019), pp. 830-838. issn: 13674811 (Electronic). doi: 10.1093/bioinformatics/bty736.

[187] Fred Glover. "Future paths for integer programming and links to artificial intelligence". In: Computers and Operations Research 13.5 (1986), pp. 533-549. issn: 03050548. doi: 10.1016/0305-0548 (86) 90048-1.

[188] Martin Pincus. "A Monte Carlo Method for the Approximate Solution of Certain Types of Constrained Optimization Problems". In: Operations Research 18.6 (Dec. 1970), pp. 1225-1228. issn: 0030-364X. doi: 10.1287/opre.18.6.1225.

[189] S. Kirkpatrick, C. D. Gelatt, and M. P. Vecchi. "Optimization by simulated annealing”. In: Science 220.4598 (May 1983), pp. 671-680. issn: 00368075. doi: 10.1126/science.220.4598.671.

[190] Fred Glover. "HEURISTICS FOR INTEGER PROGRAMMING USING SURROGATE CONSTRAINTS”. In: Decision Sciences 8.1 (Jan. 1977), pp. 156-166. issn: 15405915. doi: $10.1111 /$ j . 1540-5915 . 1977.tb01074.x.

[191] Fred Glover, Fred Glover, Manuel Laguna, and Rafael Martí. "Fundamentals of scatter search and path relinking". In: CONTROL AND CYBERNETICS 39 (2000), pp. 653-684. url: http : / citeseerx . ist.psu. edu/viewdoc/summary?doi=10.1.1.113.7217. 
[192] Jose A. Egea, Rafael Martí, and Julio R. Banga. "An evolutionary method for complex-process optimization". In: Computers and Operations Research 37.2 (Feb. 2010), pp. 315-324. issn: 03050548. doi: 10.1016/j.cor.2009.05.003.

[193] Jose A Egea, David Henriques, Thomas Cokelaer, Alejandro F Villaverde, Aidan MacNamara, Diana-Patricia Danciu, Julio R Banga, and Julio Saez-Rodriguez. "MEIGO: an open-source software suite based on metaheuristics for global optimization in systems biology and bioinformatics.” In: BMC Bioinformatics 15 (May 2014), p. 136. issn: 1471-2105 (Electronic); 1471-2105 (Linking). doi: 10 . 1186 / 1471-2105-15-136.

[194] J. A. Nelder and R. Mead. "A Simplex Method for Function Minimization”. In: The Computer Journal 7.4 (Jan. 1965), pp. 308-313. issn: 0010-4620. doi: 10.1093/comjnl/7.4.308.

[195] Karl Pearson. “ X. On the criterion that a given system of deviations from the probable in the case of a correlated system of variables is such that it can be reasonably supposed to have arisen from random sampling ". In: The London, Edinburgh, and Dublin Philosophical Magazine and Journal of Science 50.302 (July 1900), pp. 157-175. issn: 1941-5982. doi: 10.1080/14786440009463897.

[196] William G. Cochran. "The $\chi^{2}$ Test of Goodness of Fit". In: Annals of Mathematical Statistics 23.3 (1952), pp. 315-345. issn: 0003-4851. doi: 10.1214/AOMS/1177729380.

[197] Rikard Johansson, Peter Strålfors, and Gunnar Cedersund. "Combining test statistics and models in bootstrapped model rejection: It is a balancing act”. In: BMC Systems Biology 8.1 (Apr. 2014). issn: 17520509. doi: 10.1186/1752-0509-8-46.

[198] Gunnar Cedersund. "Conclusions via unique predictions obtained despite unidentifiability-new definitions and a general method." In: FEBS J 279.18 (Sept. 2012), pp. 3513-3527. issn: 1742-4658 (Electronic); 1742-464X (Linking). doi: 10 .1111/j . 1742-4658 . 2012 . 08725.x.

[199] Alexandre Sedoglavic. "A Probabilistic Algorithm to Test Local Algebraic Observability in Polynomial Time”. In: Journal of Symbolic Computation 33.5 (2002), pp. 735-755. issn: 0747-7171. doi: https: / / doi . org / 10 . 1006 / jsco . 2002 . 0532. url: http : / / www . sciencedirect.com/science/article/pii/S0747717102905328.

[200] Clemens Kreutz, Andreas Raue, Daniel Kaschek, and Jens Timmer. "Profile likelihood in systems biology." eng. In: The FEBS journal 280.11 (June 2013), pp. 2564-2571. issn: 1742-4658 (Electronic). doi: $10.1111 /$ febs. 12276 . 
[201] Jay I. Myung and Daniel J. Navarro. "Information Matrix". In: Encyclopedia of Statistics in Behavioral Science. Chichester, UK: John Wiley \& Sons, Ltd, Oct. 2005. doi: 10 . 1002/0470013192 . bsa302. url: http://doi.wiley.com/10.1002/0470013192.bsa302.

[202] Lennart. Ljung. System identification : theory for the user. English. Upper Saddle River, NJ: Prentice Hall PTR, 2012. isbn: 0136566952 9780136566953.

[203] M. Joshi, A. Seidel-Morgenstern, and A. Kremling. "Exploiting the bootstrap method for quantifying parameter confidence intervals in dynamical systems". In: Metabolic Engineering 8.5 (Sept. 2006), pp. 447-455. issn: 10967176. doi: 10.1016/j . ymben. 2006.04.003.

[204] Tim Maiwald, Helge Hass, Bernhard Steiert, Joep Vanlier, Raphael Engesser, Andreas Raue, Friederike Kipkeew, Hans H Bock, Daniel Kaschek, Clemens Kreutz, and Jens Timmer. "Driving the Model to Its Limit: Profile Likelihood Based Model Reduction.” In: PLoS One 11.9 (2016), e0162366. issn: 1932-6203 (Electronic); 1932-6203 (Linking). doi: 10.1371/journal.pone.0162366.

[205] A Raue, B Steiert, M Schelker, C Kreutz, T Maiwald, H Hass, J Vanlier, C Tönsing, L Adlung, R Engesser, W Mader, T Heinemann, J Hasenauer, M Schilling, T Höfer, E Klipp, F Theis, U Klingmüller, B Schöberl, and J Timmer. "Data2Dynamics: a modeling environment tailored to parameter estimation in dynamical systems." eng. In: Bioinformatics (Oxford, England) 31.21 (Nov. 2015), pp. 35583560. issn: 1367-4811 (Electronic). doi: 10.1093/bioinformatics/ btv405.

[206] R Boiger, J Hasenauer, S Hroß, and B Kaltenbacher. "Integration based profile likelihood calculation for PDE constrained parameter estimation problems". In: Inverse Problems 32.12 (2016), p. 125009. issn: 0266-5611. doi: 10.1088/0266-5611/32/12/125009. url: http: //dx.doi.org/10.1088/0266-5611/32/12/125009.

[207] Paul Stapor, Fabian Fröhlich, and Jan Hasenauer. "Optimization and profile calculation of ODE models using second order adjoint sensitivity analysis." eng. In: Bioinformatics (Oxford, England) 34.13 (July 2018), pp. i151-i159. issn: 1367-4811 (Electronic). doi: 10 . 1093/bioinformatics/bty230.

[208] Paul Stapor, Daniel Weindl, Benjamin Ballnus, Sabine Hug, Carolin Loos, Anna Fiedler, Sabrina Krause, Sabrina Hross, Fabian Frohlich, Jan Hasenauer, and Jonathan Wren. "PESTO: Parameter EStimation TOolbox." In: Bioinformatics 34.4 (Feb. 2018), pp. 705-707. issn: 1367-4811 (Electronic); 1367-4803 (Linking). doi: 10 . 1093/ bioinformatics/btx676. 
[209] A. Raue, C. Kreutz, T. Maiwald, U. Klingmuller, and J. Timmer. "Addressing parameter identifiability by model-based experimentation”. In: IET Systems Biology 5.2 (Mar. 2011), pp. 120-130. issn: 17518849. doi: 10.1049/iet-syb.2010.0061.

[210] Nicholas Metropolis, Arianna W. Rosenbluth, Marshall N. Rosenbluth, Augusta H. Teller, and Edward Teller. "Equation of state calculations by fast computing machines". In: The Journal of Chemical Physics 21.6 (June 1953), pp. 1087-1092. issn: 00219606. doi: 10. 1063/1 . 1699114. url: http : / aip. scitation .org/doi / 10 . 1063/1.1699114.

[211] W. K. Hastings. "Monte Carlo Sampling Methods Using Markov Chains and Their Applications". In: Biometrika 57.1 (Apr. 1970), p. 97. issn: 00063444. doi: 10 . 2307 / 2334940. url: https : / / www . jstor.org/stable/2334940.

[212] Heikki Haario, Eero Saksman, and Johanna Tamminen. "An adaptive Metropolis algorithm”. In: Bernoulli 7.2 (2001), pp. 223-242. issn: 13507265. doi: 10 . 2307 / 3318737. url: https : / /ww . jstor . org / stable/3318737.

[213] Radford M. Neal. "Sampling from multimodal distributions using tempered transitions". In: Statistics and Computing 6.4 (1996), pp. 353-366. issn: 09603174. doi: 10.1007/BF00143556.

[214] Benjamin Ballnus, Steffen Schaper, Fabian J Theis, and Jan Hasenauer. "Bayesian parameter estimation for biochemical reaction networks using region-based adaptive parallel tempering." In: Bioinformatics 34.13 (July 2018), pp. i494-i501. issn: 1367-4811 (Electronic); 1367-4803 (Linking). doi: 10.1093/bioinformatics/bty229.

[215] Mikael F. Forsgren, Markus Karlsson, Olof Dahlqvist Leinhard, Nils Dahlström, Bengt Norén, Thobias Romu, Simone Ignatova, Mattias Ekstedt, Stergios Kechagias, Peter Lundberg, and Gunnar Cedersund. "Model-inferred mechanisms of liver function from magnetic resonance imaging data: Validation and variation across a clinically relevant cohort". In: PLOS Computational Biology 15.6 (June 2019). Ed. by James Gallo, e1007157. issn: 1553-7358. doi: 10.1371/ journal . pcbi . 1007157. url: https : / / dx . plos . org/10 . 1371/ journal.pcbi.1007157.

[216] Nicolas Sundqvist, Roland Nilsson, and Gunnar Cedersund. "Advantages of using validation for selecting the appropriate $13 \mathrm{C}$ metabolic flux model: theory, examples, and a general method". In: Manuscript (2020). 
[217] Tommaso Piccoli, Giancarlo Valente, David E J Linden, Marta Re, Fabrizio Esposito, Alexander T Sack, and Francesco Di Salle. "The default mode network and the working memory network are not anticorrelated during all phases of a working memory task." In: PLoS One 10.4 (2015), e0123354. issn: 1932-6203 (Electronic); 1932-6203 (Linking). doi: 10.1371/journal . pone.0123354.

[218] M E Raichle, A M MacLeod, A Z Snyder, W J Powers, D A Gusnard, and G L Shulman. "A default mode of brain function." In: Proc Natl Acad Sci U S A 98.2 (Jan. 2001), pp. 676-682. issn: 0027-8424 (Print); 0027-8424 (Linking). doi: 10.1073/pnas .98.2.676.

[219] Marta Ceko, John L Gracely, Mary-Ann Fitzcharles, David A Seminowicz, Petra Schweinhardt, and M Catherine Bushnell. "Is a Responsive Default Mode Network Required for Successful Working Memory Task Performance?" In: J Neurosci 35.33 (Aug. 2015), pp. 1159511605. issn: 1529-2401 (Electronic); 0270-6474 (Linking). doi: 10 . 1523/JNEUROSCI . 0264-15. 2015.

[220] Susan Whitfield-Gabrieli, Heidi W Thermenos, Snezana Milanovic, Ming T Tsuang, Stephen V Faraone, Robert W McCarley, Martha E Shenton, Alan I Green, Alfonso Nieto-Castanon, Peter LaViolette, Joanne Wojcik, John D E Gabrieli, and Larry J Seidman. "Hyperactivity and hyperconnectivity of the default network in schizophrenia and in first-degree relatives of persons with schizophrenia." In: Proc Natl Acad Sci U S A 106.4 (Jan. 2009), pp. 1279-1284. issn: 1091-6490 (Electronic); 0027-8424 (Linking). doi: 10.1073/pnas . 0809141106.

[221] Amir Shmuel, Mark Augath, Axel Oeltermann, and Nikos K Logothetis. "Negative functional MRI response correlates with decreases in neuronal activity in monkey visual area V1." In: Nat Neurosci 9.4 (Apr. 2006), pp. 569-577. issn: 1097-6256 (Print); 1097-6256 (Linking). doi: $10.1038 / \mathrm{nn} 1675$.

[222] K. J. Mullinger, S. D. Mayhew, A. P. Bagshaw, R. Bowtell, and S. T. Francis. "Evidence that the negative BOLD response is neuronal in origin: A simultaneous EEG-BOLD-CBF study in humans". In: NeuroImage 94 (2014), pp. 263-274. issn: 10959572. doi: 10.1016/ j . neuroimage. 2014.02.029.

[223] Jozien Goense, Yvette Bohraus, and Nikos K. Logothetis. "fMRI at high spatial resolution implications for BOLD-models". In: Frontiers in Computational Neuroscience 10.Jun (2016), p. 66. issn: 16625188. doi: 10.3389/fncom.2016.00066.

[224] Noam Harel, Sang-Pil Lee, Tsukasa Nagaoka, Dae-Shik Kim, and Seong-Gi Kim. "Origin of Negative Blood Oxygenation LevelDependent fMRI Signals". In: Journal of Cerebral Blood Flow \& 
Metabolism 22.8 (Aug. 2002), pp. 908-917. issn: 0271-678. doi: 10 . 1097/00004647-200208000-00002.

[225] Sridhar S. Kannurpatti and Bharat B. Biswal. "Negative functional response to sensory stimulation and its origins". In: Journal of Cerebral Blood Flow and Metabolism 24.6 (2004), pp. 703-712. issn: 0271678X. doi: 10.1097/01.WCB.0000121232.04853.46.

[226] Anna Devor, Elizabeth M. C. Hillman, Peifang Tian, Christian Waeber, Ivan C. Teng, Lana Ruvinskaya, Mark H. Shalinsky, Haihao Zhu, Robert H. Haslinger, Suresh N. Narayanan, Istvan Ulbert, Andrew K. Dunn, Eng H. Lo, Bruce R. Rosen, Anders M. Dale, David Kleinfeld, and David A. Boas. "Stimulus-induced changes in blood flow and 2deoxyglucose uptake dissociate in ipsilateral somatosensory cortex." In: $J$ Neurosci 28.53 (Dec. 2008), pp. 14347-14357. issn: 1529-2401 (Electronic); 0270-6474 (Linking). doi: 10 . 1523/ JNEUROSCI . 430708.2008.

[227] György Buzsáki, Kai Kaila, and Marcus Raichle. "Inhibition and brain work.” In: Neuron 56.5 (2007), pp. 771-783. issn: 0896-6273. doi: $10.1016 / j$. neuron. 2007.11.008.

[228] Valerie E.M. Griffeth and Richard B. Buxton. "A theoretical framework for estimating cerebral oxygen metabolism changes using the calibrated-BOLD method: Modeling the effects of blood volume distribution, hematocrit, oxygen extraction fraction, and tissue signal properties on the BOLD signal". In: NeuroImage 58.1 (2011), pp. 198-212. issn: 10538119. doi: 10 . 1016/j . neuroimage . 2011 . 05.077 .

[229] Jean J. Chen and G. Bruce Pike. "Origins of the BOLD post-stimulus undershoot”. In: NeuroImage 46.3 (July 2009), pp. 559-568. issn: 10538119. doi: $10.1016 / \mathrm{j}$. neuroimage . 2009 . 03 .015. url: https : //pubmed.ncbi.nlm.nih.gov/19303450/.

[230] Peter C.M. van Zijl, Jun Hua, and Hanzhang Lu. The BOLD poststimulus undershoot, one of the most debated issues in fMRI. Aug. 2012. doi: 10 . $1016 / \mathrm{j}$. neuroimage . 2012 . 01 . 029. url: /pmc/ articles/PMC3356682/?report=abstract\%20https : //www . ncbi . nlm.nih.gov/pmc/articles/PMC3356682/.

[231] Gaute T. Einevoll, Alain Destexhe, Markus Diesmann, Sonja Grün, Viktor Jirsa, Marc de Kamps, Michele Migliore, Torbjørn V. Ness, Hans E. Plesser, and Felix Schürmann. The Scientific Case for Brain Simulations. May 2019. doi: 10.1016/j.neuron. 2019.03.027. 
[232] Suzanne T Witt, Marcel Warntjes, and Maria Engström. "Increased fMRI Sensitivity at Equal Data Burden Using Averaged Shifted Echo Acquisition". In: Frontiers in Neuroscience 10 (2016), p. 544. issn: 1662-453X. doi: 10.3389/fnins. 2016.00544. url: http://journal. frontiersin.org/article/10.3389/fnins.2016.00544.

[233] Susanna A Walter, Mikael Forsgren, Karin Lundengård, Rozalyn Simon, Maritha Torkildsen Nilsson, Birgitta Söderfeldt, Peter Lundberg, and Maria Engström. "Positive Allosteric Modulator of GABA Lowers BOLD Responses in the Cingulate Cortex." In: PLoS ONE 11.3 (2016), e0148737. issn: 1932-6203. doi: 10 . 1371/ journal . pone . 0148737.

[234] Mattias Ragnehed, Irene Håkansson, Maritha Nilsson, Peter Lundberg, Birgitta Söderfeldt, and Maria Engström. "Influence of diazepam on clinically designed FMRI." In: $J$ Neuropsychiatry Clin Neurosci 19.2 (2007), pp. 164-172. issn: 0895-0172. doi: 10.1176/ jnp. 2007.19.2.164.

[235] Christian Szegedy, Alexander Toshev, and Dumitru Erhan. Deep Neural Networks for Object Detection. Tech. rep.

[236] Daniel Bullock and Stephen Grossberg. "Adaptive neural networks for control of movement trajectories invariant under speed and force rescaling". In: Human Movement Science 10.1 (Feb. 1991), pp. 3-53. issn: 01679457. doi: 10.1016/0167-9457 (91) 90029-W.

[237] Stephen Grossberg. "Adaptive Resonance Theory: How a brain learns to consciously attend, learn, and recognize a changing world". In: Neural Networks 37 (Jan. 2013), pp. 1-47. issn: 08936080. doi: 10. 1016/j.neunet.2012.09.017.

[238] Mark Sagar, David Bullivant, Oleg Efimov, Muhammad Jawed, Ratheesh Kalarot, Paul Robertson, and Tim Wu. "Embodying models of expressive behaviour and learning with a biomimetic virtual infant". In: IEEE ICDL-EPIROB 2014 - 4th Joint IEEE International Conference on Development and Learning and on Epigenetic Robotics. Institute of Electrical and Electronics Engineers Inc., Dec. 2014, pp. 62-67. isbn: 9781479975402. doi: 10.1109/DEVLRN. 2014. 6982955.

[239] Mark Sagar. "BabyX". In: ACM SIGGRAPH 2015 Computer Animation Festival. New York, NY, USA: Association for Computing Machinery (ACM), July 2015, pp. 184-184. doi: 10 . 1145/ 2790329 . 2790330. url: http://dl . acm.org/doi/10.1145/2790329.2790330. 
[240] FDA. Reporting of Computational Modeling Studies in Medical Device Submissions. 2016. url: https : / / www . fda . gov / regulatory - information / search - fda-guidance-documents / reporting-computational-modeling-studies-medical-devicesubmissions.

[241] Tina M. Morrison, Pras Pathmanathan, Mariam Adwan, and Edward Margerrison. "Advancing regulatory science with computational modeling for medical devices at the FDA's office of science and engineering laboratories". In: Frontiers in Medicine 5.SEP (2018). issn: 2296858X. doi: 10 . 3389 / fmed . 2018 . 00241. url: /pmc / articles/PMC6167449/?report=abstract\%20https : //www . ncbi . nlm.nih.gov/pmc/articles/PMC6167449/.

[242] Marco Viceconti, Francesco Pappalardo, Blanca Rodriguez, Marc Horner, Jeff Bischoff, and Flora Musuamba Tshinanu. "In silico trials: Verification, validation and uncertainty quantification of predictive models used in the regulatory evaluation of biomedical products”. In: Methods (Jan. 2020). issn: 10959130. doi: 10 . 1016 / j . ymeth.2020.01.011. 


\section{Papers}

The papers associated with this thesis have been removed for copyright reasons. For more details about these see:

http://urn.kb.se/resolve?urn=urn:nbn:se:liu:diva-167806 


\section{FACULTY OF MEDICINE AND HEALTH SCIENCES}

Linköping University Medical Dissertation No. 1742, 2020

Department of Health, Medicine and Caring Sciences

Division of Diagnostics and Specialist Medicine

Linköping University

SE-581 83 Linköping, Sweden

www.liu.se 